\title{
Cyclization or Hydrogen Migration: Theoretical Study and Experimental Evidence on the Reactivities of Unsaturated Amidyl Radicals
}

\author{
Qian Chen, Meihua Shen, Yu Tang and Chaozhong Li*
}

Shanghai Institute of Organic Chemistry, Chinese Academy of Sciences, 354 Fenglin

Road, Shanghai 200032, P. R. China

clig@mail.sioc.ac.cn

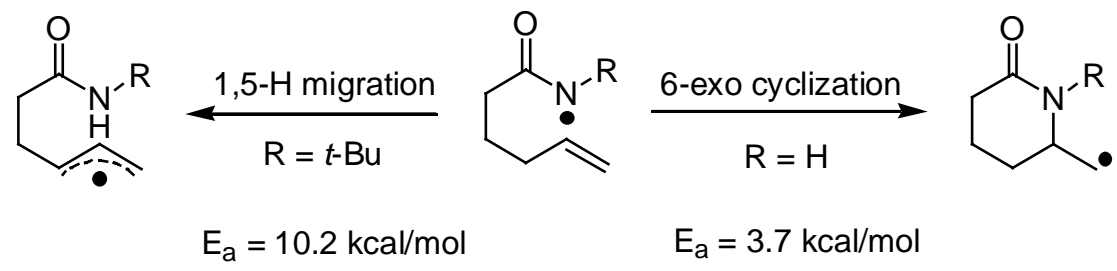

Supporting Information 


\section{List of Contents}

1. Synthesis and Characterizations of 4-5 and 7-9.

2. Table S1. Computational (UB3LYP/6-31G*) Results on Radicals 1.

3. Table S2. Computational (UB3LYP/6-31G*) Results on the Transition States of 6-Exo Cyclization of $\mathbf{1}$.

4. Table S3. Computational (UB3LYP/6-31G*) Results on the Transition States of 1,5-H Abstraction of $\mathbf{1}$.

5. Table S4. Computational (UB3LYP/6-31G*) Results on Radicals 2 and 3.

6. Table S5. Computational (UB3LYP/6-31G*) Results on the Transition State of 7-Endo Cyclization of $\mathbf{1}(\mathrm{R}=\mathrm{H})$.

7. Table S6. Computational (UB3LYP/6-31G*) Results on Radicals 11.

8. Table S7. Computational (UB3LYP/6-31G*) Results on the Transition States of Cyclization of 11.

9. Table S8. Computational (UB3LYP/6-31G*) Results on the Transition States of 1,5-H Abstraction of $\mathbf{1 1}$.

10. Table S9. Computational (UB3LYP/6-31G*) Results on the Transition States of 1,6-H Abstraction of $\mathbf{1 1 .}$

11. Table S10. ${ }^{1} \mathrm{H}$ or ${ }^{13} \mathrm{C}$ NMR Spectra of $4,5,7$ and 9. 
$N$-tert-Butyl- $N$-(phenylthio)hex-5-enamide (4). To a solution of $N$-phenylthio-tert-butylamine ${ }^{[1]}(1.81 \mathrm{~g}, 10 \mathrm{mmol})$ in dry THF $(20 \mathrm{~mL})$ was added BuLi ( $2 \mathrm{M}$ in cyclohexane, $5 \mathrm{~mL}, 10 \mathrm{mmol})$ at $-78{ }^{\circ} \mathrm{C}$ under nitrogen atmosphere. The mixture was stirred at $-78{ }^{\circ} \mathrm{C}$ for $0.5 \mathrm{~h}$. Hex-5-enoyl chloride $(1.31 \mathrm{~mL}, 10 \mathrm{mmol})$ in dry THF $(10 \mathrm{~mL})$ was added. The resulting mixture was stirred at $-78{ }^{\circ} \mathrm{C}$ for $20 \mathrm{~min}$ and was then allowed to warm up to room temperature and stirred for an additional $2 \mathrm{~h}$. Water $(15 \mathrm{~mL})$ was added. The two layers were separated and the aqueous phase was extracted with ethyl acetate $(3 \times 30 \mathrm{~mL})$. The combined organic phase was washed with brine $(2 \times 15 \mathrm{~mL})$ and then dried over anhydrous $\mathrm{MgSO}_{4}$. After removal of the solvent, the crude product was purified by column chromatography on silica gel with hexane-ethyl acetate (50:1, v:v) as the eluent to give $\mathbf{4}$ as a colorless oil. Yield: $1.25 \mathrm{~g}$ (45\%). ${ }^{1} \mathrm{H}$ NMR $\left(300 \mathrm{MHz}, \mathrm{CDCl}_{3}\right) \delta 1.50(9 \mathrm{H}, \mathrm{s}), 1.67-1.77(2 \mathrm{H}, \mathrm{m}), 2.01-2.09(2 \mathrm{H}$, $\mathrm{m}), 2.41-2.52(1 \mathrm{H}, \mathrm{m}), 2.88-2.98(1 \mathrm{H}, \mathrm{m}), 4.93-5.01(2 \mathrm{H}, \mathrm{m}), 5.71-5.80(1 \mathrm{H}, \mathrm{m})$, 7.11-7.19 (3H, m), 7.30-7.36 (2H, m); ${ }^{13} \mathrm{C} \mathrm{NMR}\left(\mathrm{CDCl}_{3}\right) \delta 24.8,29.3,33.0,35.5,63.1$, 114.7, 121.7, 125.6, 129.0, 138.1, 141.0, 179.4; EIMS: $\mathrm{m} / \mathrm{z}$ (rel intensity) $276\left(\mathrm{M}^{+}-1,5\right)$, 260 (6), 220 (13), 204 (11), 177 (32), 135 (34), 109 (61), 57 (100); HRMS calcd for $\mathrm{C}_{16} \mathrm{H}_{24} \mathrm{NOS}\left(\mathrm{M}^{+}+\mathrm{H}\right): 278.1573$. Found: 278.1574 .

[1] Davis, F. A. J. Org. Chem. 1977, 42, 967.

$N$-(Phenylthio)hex-5-enamide (7). To NaH (60\% in mineral oil, $0.48 \mathrm{~g}, 12 \mathrm{mmol}$ ) in dry THF $(80 \mathrm{~mL})$ was added in portions hex-5-enamide $(1.14 \mathrm{~g}, 10 \mathrm{mmol})$ at room temperature under nitrogen atmosphere and the mixture was stirred at room temperature for $1 \mathrm{~h}$. Diphenyldisulfide $(2.62 \mathrm{~g}, 12 \mathrm{mmol})$ was added and the resulting mixture was stirred at room temperature for $12 \mathrm{~h}$. Ethyl ether $(30 \mathrm{~mL})$ was added. The residue was filtered over a plug of siliga gel and washed with ethyl ether $(200 \mathrm{~mL})$. The filtrate was concentrated and the crude product was purified by column chromatography on silica gel with hexane-ethyl acetate (10:1, v:v) as the eluent to give 7 as a white solid. Yield: $1.05 \mathrm{~g}(48 \%)$. Compound 7 existed as the mixture of two forms $7 \mathbf{a}$ and $\mathbf{7 b}$ (2:1 ratio determined by $\left.{ }^{1} \mathrm{H} \mathrm{NMR}\right) .7 \mathbf{a}:{ }^{1} \mathrm{H} \mathrm{NMR}\left(300 \mathrm{MHz}, \mathrm{CDCl}_{3}\right) \delta$ 1.76-1.86 (2H, m), 2.07-2.14 (2H, m), 2.37(2H, t, $J=7.2 \mathrm{~Hz}), 4.96-5.04(2 \mathrm{H}, \mathrm{m})$, 5.70-5.81 $(1 \mathrm{H}, \mathrm{m}), 6.85(1 \mathrm{H}, \mathrm{br}), 7.20-7.36(5 \mathrm{H}, \mathrm{m}) ;{ }^{13} \mathrm{C} \mathrm{NMR}\left(\mathrm{CDCl}_{3}\right) \delta 24.5,32.9$, 35.6, 115.4, 124.9, 126.5, 128.8, 137.5, 138.7, 175.4. 7b: ${ }^{1} \mathrm{H} \mathrm{NMR}\left(300 \mathrm{MHz}, \mathrm{CDCl}_{3}\right)$ $\delta$ 1.68-1.76 (2H, m), 2.07-2.14 (2H, m), $2.56(2 \mathrm{H}, \mathrm{t}, J=6.9 \mathrm{~Hz}), 4.96-5.04(2 \mathrm{H}, \mathrm{m})$, 5.70-5.81 (1H, m), $6.48(1 \mathrm{H}, \mathrm{br}), 7.20-7.36(5 \mathrm{H}, \mathrm{m}),{ }^{13} \mathrm{C} \mathrm{NMR}\left(\mathrm{CDCl}_{3}\right) \delta 23.6,30.1$, 35.6, 115.4, 123.1, 126.5, 129.2, 137.5, 138.7, 180.1. EIMS: $m / z$ (rel intensity) 221 $\left(\mathrm{M}^{+}, 15\right), 188$ (2), 167 (2), 150 (3), 125 (100), 109 (17), 93 (12), 69 (17); Anal. calcd for $\mathrm{C}_{12} \mathrm{H}_{15} \mathrm{NOS}$ : C, 65.12; H, 6.83; N, 6.33. Found: C, 65.14; H, 6.67; N, 6.23. 


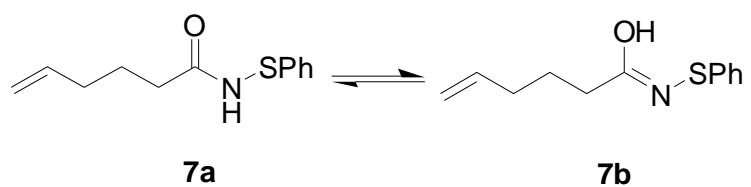

Reaction of 4. $N$-tert-butyl- $N$-(phenylthio)hex-5-enamide (4) (277 mg, $1 \mathrm{mmol}$ ) was dissolved in $80 \mathrm{~mL}$ of anhydrous benzene, and the solution was brought to reflux. Into this refluxing solution was added dropwise over a period of $0.5 \mathrm{~h} \mathrm{Bu}_{3} \mathrm{SnD}(0.54 \mathrm{~mL}, 2$ mmol) and AIBN (49 mg, $0.3 \mathrm{mmol})$ in benzene solution $(20 \mathrm{~mL})$ under nitrogen atmosphere. The mixture was then refluxed for an additional $2 \mathrm{~h}$ and cooled down to room temperature. After removal of the solvent, the residue was chromatographed on silica gel with hexane-acetone (100:1 to 5:1) as the eluent to give 5 as a mixture of two stereoisomers ( $Z: E=1.4: 1$ determined by ${ }^{1} \mathrm{H}$ NMR).Yield: $92 \mathrm{mg}(54 \%)$. The two isomers were further separated using $\mathrm{AgNO}_{3}$-containing silica gel. (Z)-5: ${ }^{1} \mathrm{H}$ NMR $\left(300 \mathrm{MHz}, \mathrm{CDCl}_{3}\right) \delta 1.33(9 \mathrm{H}, \mathrm{s}), 1.61-1.65(2 \mathrm{H}, \mathrm{m}), 2.11-2.16(2 \mathrm{H}, \mathrm{m}), 2.24-2.32(2 \mathrm{H}$, m), $5.36(1 \mathrm{H}, \mathrm{br}), 5.38-5.52(2 \mathrm{H}, \mathrm{m}) ;{ }^{13} \mathrm{C} \mathrm{NMR}\left(\mathrm{CDCl}_{3}\right) \delta 17.6(\mathrm{t}, J=19.4 \mathrm{~Hz}), 28.7$, 28.8, 37.4, 51.0, 126.1, 129.6, 171.9; EIMS: $m / z$ (rel intensity) $171\left(\mathbf{M}^{+}+1,100\right), 153$ (1), 143 (1), 115 (18), 98 (2), 71 (3), 58 (27), 42 (8); HRMS calcd for $\mathrm{C}_{10} \mathrm{H}_{18} \mathrm{DNO}$ : 170.1561. Found: 170.1545. (E)-5: ${ }^{1} \mathrm{H}$ NMR $\left(300 \mathrm{MHz}, \mathrm{CDCl}_{3}\right) \delta 1.34(9 \mathrm{H}, \mathrm{s})$, 1.61-1.63 (2H, m), 2.11-2.16 (2H, m), 2.32-2.39 (2H, m), $5.27(1 \mathrm{H}, \mathrm{br}), 5.32-5.40(1 \mathrm{H}$, m), 5.46-5.54 $(1 \mathrm{H}, \mathrm{m}) ;{ }^{13} \mathrm{C} \mathrm{NMR}\left(\mathrm{CDCl}_{3}\right) \delta 23.2,28.8,29.7,37.3,51.0,125.2,128.8$, 171.9; EIMS: $m / z$ (rel intensity) $171\left(\mathrm{M}^{+}+1,100\right), 155$ (1), 143 (1), 115 (22), 98 (6), 89 (13), 71 (9), 58 (53); HRMS calcd for $\mathrm{C}_{10} \mathrm{H}_{18}$ DNO: 170.1496. Found: 170.1513.

Reaction of 7. $N$-(Phenylthio)hex-5-enamide (7) $(222 \mathrm{mg}, 1 \mathrm{mmol})$ was dissolved in $90 \mathrm{~mL}$ of anhydrous benzene, and the solution was brought to reflux. Into this refluxing solution was added over a period of $5 \mathrm{~min} \mathrm{Bu}_{3} \mathrm{SnD}(0.49 \mathrm{~mL}, 1.8 \mathrm{mmol})$ and AIBN (49 mg, $0.3 \mathrm{mmol}$ ) in benzene solution $(10 \mathrm{~mL})$ under nitrogen atmosphere. The mixture was refluxed for an additional $1 \mathrm{~h}$ and then cooled down to room temperature. After removal of the solvent, the residue was chromatographed on silica gel with hexane-acetone (100:1 to 1:1) as the eluent to give the mixture of 8 and 9 . Yield: $91 \mathrm{mg}$ (80\%). The ratio of 8 to 9 was 4 to 1 determined by ${ }^{1}$ H NMR. 8 and 9 were then separated by further chromatography on silica gel. 8: ${ }^{1} \mathrm{H}$ NMR $\left(300 \mathrm{MHz}, \mathrm{CDCl}_{3}\right) \delta$ $1.18(2 \mathrm{H}, \mathrm{d}, J=6.0 \mathrm{~Hz}), 1.26-1.39(1 \mathrm{H}, \mathrm{m}), 1.63-1.76(1 \mathrm{H}, \mathrm{m}), 1.88-1.92(2 \mathrm{H}, \mathrm{m})$, 2.18-2.42 (2H, m), 3.47-3.52 (1H, m), $6.19(1 \mathrm{H}, \mathrm{br}) ;{ }^{13} \mathrm{C} \mathrm{NMR}\left(\mathrm{CDCl}_{3}\right) \delta 19.7,22.2(\mathrm{t}$, $J=19.5 \mathrm{~Hz}$ ), 30.2, 30.8, 48.5, 172.6; EIMS: $\mathrm{m} / z$ (rel intensity) $114\left(\mathrm{M}^{+}, 26\right), 98(100)$, 86 (4), 70 (11), 58 (18), 55 (63), 45 (60), 43 (38); HRMS calculated for $\mathrm{C}_{6} \mathrm{H}_{11} \mathrm{DNO}$ $\left(\mathrm{M}^{+}+\mathrm{H}\right)$ : 115.0976. Found: 115.0982. 9: ${ }^{1} \mathrm{H}$ NMR $\left(300 \mathrm{MHz}, \mathrm{CDCl}_{3}\right) \delta 1.59-1.72(3 \mathrm{H}$, $\mathrm{m}), 1.84-1.87(2 \mathrm{H}, \mathrm{m}), 2.41-2.44(2 \mathrm{H}, \mathrm{m}), 3.15-3.18(2 \mathrm{H}, \mathrm{m}), 7.11(1 \mathrm{H}, \mathrm{br}) ;{ }^{13} \mathrm{C} \mathrm{NMR}$ $\left(\mathrm{CDCl}_{3}\right) \delta 23.1,29.2(\mathrm{t}, J=19.5 \mathrm{~Hz}), 30.4,36.6,42.6,179.4$. Anal. calcd for $\mathrm{C}_{6} \mathrm{H}_{10}$ DNO: $\mathrm{C}, 63.12 ; \mathrm{H}, 8.83 ; \mathrm{N}, 12.27$. Found: $\mathrm{C}, 62.94 ; \mathrm{H}, 9.15 ; \mathrm{N}, 12.10$.

Table S1. Computational (UB3LYP/6-31G*) Results on Radicals 1. 

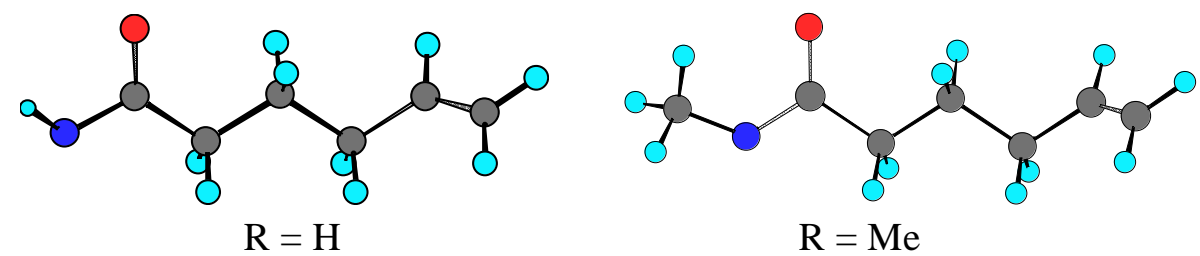

$\mathrm{R}=\mathrm{Me}$

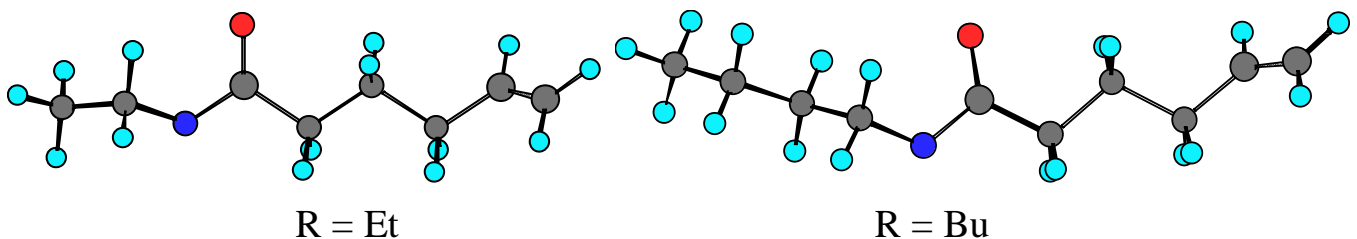

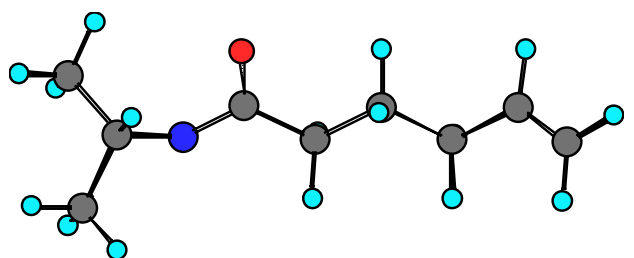

$\mathrm{R}=i-\operatorname{Pr}$

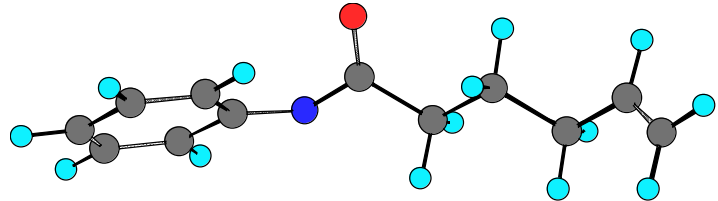

$\mathrm{R}=\mathrm{Ph}$

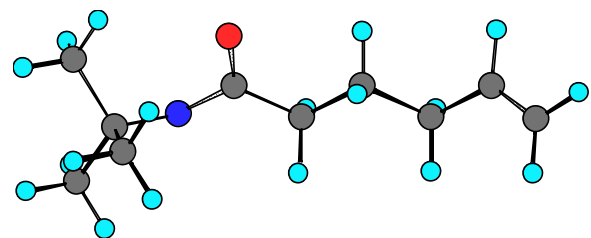

$\mathrm{R}=t-\mathrm{Bu}$

$$
\mathrm{R}=\mathrm{OMe}
$$

$\% \operatorname{chk}=1(\mathrm{R}=\mathrm{H})$

\# ub3lyp/6-31g* opt freq=noraman

calculate for $1 \quad \mathrm{HF}=-364.550773$

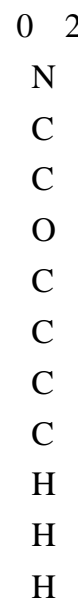

$\begin{array}{llll}1.375942 & & & \\ 1.512087 & 1 & 117.060097 & \\ 1.237719 & 1 & 118.522727 & 3 \\ 1.530173 & 2 & 113.759736 & 1 \\ 1.539900 & 3 & 112.383976 & 2 \\ 1.504731 & 5 & 112.644836 & 3 \\ 1.333263 & 6 & 125.249280 & 5 \\ 1.098739 & 2 & 107.509798 & 1 \\ 1.028586 & 2 & 108.345899 & 4 \\ 1.100155 & 2 & 107.508641 & 1\end{array}$

172.893308

177.983433

179.661259

177.978078

119.645713

54.249682

$-30.619247$

$-58.480546$ 


$\begin{array}{rrrrrrr}\mathrm{H} & 5 & 1.096649 & 3 & 109.739121 & 2 & 57.552260 \\ \mathrm{H} & 5 & 1.096666 & 3 & 109.123806 & 2 & -58.110118 \\ \mathrm{H} & 6 & 1.098338 & 5 & 109.891213 & 3 & -59.470327 \\ \mathrm{H} & 6 & 1.101399 & 5 & 108.659726 & 3 & 56.678541 \\ \mathrm{H} & 8 & 1.086731 & 7 & 121.832194 & 6 & -179.654360 \\ \mathrm{H} & 8 & 1.088518 & 7 & 121.693260 & 6 & 0.572805 \\ \mathrm{H} & 7 & 1.091656 & 6 & 115.682227 & 5 & -59.672734\end{array}$

$\% \operatorname{chk}=1(\mathrm{R}=\mathrm{Me})$

\# ub31yp/6-31g* opt freq=noraman

calculate for $1 \quad \mathrm{HF}=-403.869789$

$\begin{array}{ll}0 & 2\end{array}$

$\mathrm{N}$

$\mathrm{C}$

1.389196

C

$\mathrm{O}$

$\mathrm{C}$

C

C

C

C

$\mathrm{H}$

$\mathrm{H}$

$\mathrm{H}$

$\mathrm{H}$

$\mathrm{H}$

$\mathrm{H}$

$\mathrm{H}$

$\mathrm{H}$

$\mathrm{H}$

$\mathrm{H}$

$\mathrm{H}$

$\mathrm{H}$

1
2
2
3
5
6
7
1
3
3
5
5
6
6
8
8
7
9
9
9

1.517390

1.229826

$1 \quad 114.647146$

$1 \quad 121.26091$

$1.529818 \quad 2$

113.502677

$1.539984 \quad 3$

$112.434288 \quad 2$

$\begin{array}{llll}1.504772 & 5 & 112.676736 & 3\end{array}$

$\begin{array}{llll}1.333303 & 6 & 125.246535 & 5\end{array}$

$\begin{array}{llll}1.439503 & 2 & 117.213688 & 4\end{array}$

$\begin{array}{llll}1.099267 & 2 & 107.401237 & 1\end{array}$

$\begin{array}{lll}1.099901 & 2 & 108.095771\end{array}$

$\begin{array}{llll}1.096676 & 3 & 109.723768 & 2\end{array}$

$\begin{array}{llll}1.096445 & 3 & 109.040682 & 2\end{array}$

$\begin{array}{llll}1.098377 & 5 & 109.933138 & 3\end{array}$

$\begin{array}{llll}1.101438 & 5 & 108.641171 & 3\end{array}$

$\begin{array}{llll}1.086769 & 7 & 121.866583 & 6\end{array}$

$\begin{array}{llll}1.088526 & 7 & 121.655591 & 6\end{array}$

$\begin{array}{llll}1.091710 & 6 & 115.695715 & 5\end{array}$

$\begin{array}{llll}1.097595 & 1 & 109.342032 & 2\end{array}$

$\begin{array}{llll}1.101648 & 1 & 107.632002 & 2\end{array}$

1.095920

$113.602456 \quad 2$

174.358564

$-176.802511$

$-178.953867$

178.356163

119.596126

$-34.720465$

59.991429

$-52.885250$

58.920006

$-56.639473$

$-59.081392$

57.046284

$-179.599436$

0.608999

$-59.652231$

127.509431

$-117.765808$

3.860720

\%chk=1 $(\mathrm{R}=\mathrm{Et})$

\# ub3lyp/6-31g* opt freq=noraman

calculate for 1

$\mathrm{HF}=-443.186534$

$0 \quad 2$ 


$\begin{array}{lrrrrrr}\mathrm{N} & & & & & \\ \mathrm{C} & 1 & 1.389189 & & & & \\ \mathrm{C} & 2 & 1.518519 & 1 & 114.602593 & & \\ \mathrm{O} & 2 & 1.229013 & 1 & 121.346029 & 3 & 174.249255 \\ \mathrm{C} & 3 & 1.529903 & 2 & 113.440080 & 1 & -176.610476 \\ \mathrm{C} & 5 & 1.540023 & 3 & 112.442941 & 2 & -179.024371 \\ \mathrm{C} & 6 & 1.504753 & 5 & 112.653659 & 3 & 178.310364 \\ \mathrm{C} & 7 & 1.333309 & 6 & 125.267942 & 5 & 119.520368 \\ \mathrm{C} & 1 & 1.443563 & 2 & 117.643564 & 4 & -39.887939 \\ \mathrm{C} & 9 & 1.530206 & 1 & 111.747790 & 2 & 140.638275 \\ \mathrm{H} & 3 & 1.099304 & 2 & 107.369542 & 1 & 60.245027 \\ \mathrm{H} & 3 & 1.099939 & 2 & 108.185384 & 1 & -52.689328 \\ \mathrm{H} & 5 & 1.096609 & 3 & 109.715491 & 2 & 58.852234 \\ \mathrm{H} & 5 & 1.096392 & 3 & 109.026859 & 2 & -56.692209 \\ \mathrm{H} & 6 & 1.098364 & 5 & 109.935812 & 3 & -59.138595 \\ \mathrm{H} & 6 & 1.101436 & 5 & 108.654250 & 3 & 57.006387 \\ \mathrm{H} & 8 & 1.086777 & 7 & 121.858899 & 6 & -179.577536 \\ \mathrm{H} & 8 & 1.088536 & 7 & 121.671723 & 6 & 0.618142 \\ \mathrm{H} & 7 & 1.091724 & 6 & 115.685964 & 5 & -59.700181 \\ \mathrm{H} & 9 & 1.105287 & 1 & 106.210493 & 2 & -100.358162 \\ \mathrm{H} & 9 & 1.098871 & 1 & 110.704263 & 2 & 15.230823 \\ \mathrm{H} & 10 & 1.095609 & 9 & 110.216968 & 1 & 175.851143 \\ \mathrm{H} & 10 & 1.094526 & 9 & 110.498391 & 1 & -64.478051 \\ \mathrm{H} & 10 & 1.094449 & 9 & 110.845414 & 1 & 55.510880 \\ & & & & & & \end{array}$

$\% \operatorname{chk}=1(\mathrm{R}=\mathrm{Bu})$

\# ub3lyp/6-31g* opt freq=noraman

calculate for $1 \quad \mathrm{HF}=-521.814446$

$\begin{array}{lrrrrrr}0 \quad 2 & & & & \\ \mathrm{~N} & 1 & 1.384277 & & & & \\ \mathrm{C} & 2 & 1.517291 & 1 & 115.193390 & & \\ \mathrm{C} & 2 & 1.231876 & 1 & 120.811266 & 3 & 174.413274 \\ \mathrm{O} & 3 & 1.530028 & 2 & 113.496449 & 1 & -176.603832 \\ \mathrm{C} & 5 & 1.540013 & 3 & 112.449376 & 2 & -179.258900 \\ \mathrm{C} & 6 & 1.504757 & 5 & 112.672289 & 3 & 178.501443 \\ \mathrm{C} & 7 & 1.333309 & 6 & 125.266218 & 5 & 119.656107 \\ \mathrm{C} & 1 & 1.442719 & 2 & 117.378140 & 4 & -33.112716 \\ \mathrm{C} & 9 & 1.546623 & 1 & 110.394748 & 2 & 105.276338 \\ \mathrm{C} & 10 & 1.533636 & 9 & 112.146017 & 1 & 174.832837 \\ \mathrm{C} & 11 & 1.532410 & 10 & 112.554748 & 9 & 178.541575 \\ \mathrm{C} & & & & & & \end{array}$




$\begin{array}{lrrrrrr}\mathrm{H} & 3 & 1.099279 & 2 & 107.437992 & 1 & 60.197718 \\ \mathrm{H} & 3 & 1.099839 & 2 & 108.080268 & 1 & -52.739871 \\ \mathrm{H} & 5 & 1.096683 & 3 & 109.696342 & 2 & 58.642246 \\ \mathrm{H} & 5 & 1.096427 & 3 & 109.079852 & 2 & -56.918687 \\ \mathrm{H} & 6 & 1.098341 & 5 & 109.914978 & 3 & -58.929415 \\ \mathrm{H} & 6 & 1.101475 & 5 & 108.652335 & 3 & 57.202559 \\ \mathrm{H} & 8 & 1.086786 & 7 & 121.866239 & 6 & -179.596528 \\ \mathrm{H} & 8 & 1.088512 & 7 & 121.659007 & 6 & 0.591108 \\ \mathrm{H} & 7 & 1.091734 & 6 & 115.688474 & 5 & -59.590389 \\ \mathrm{H} & 9 & 1.098055 & 1 & 112.611843 & 2 & -17.775130 \\ \mathrm{H} & 10 & 1.097033 & 9 & 108.830923 & 1 & 52.389422 \\ \mathrm{H} & 10 & 1.096456 & 9 & 107.925832 & 1 & -63.615476 \\ \mathrm{H} & 11 & 1.099183 & 10 & 109.405775 & 9 & -59.425391 \\ \mathrm{H} & 11 & 1.098699 & 10 & 109.404132 & 9 & 56.630508 \\ \mathrm{H} & 9 & 1.100242 & 1 & 106.636755 & 2 & -137.407867 \\ \mathrm{H} & 12 & 1.096705 & 11 & 111.226044 & 10 & 59.962975 \\ \mathrm{H} & 12 & 1.095848 & 11 & 111.185822 & 10 & -179.954667 \\ \mathrm{H} & 12 & 1.096533 & 11 & 111.146333 & 10 & -59.934126\end{array}$

$\%$ chk=1 $(\mathrm{R}=i$-Pr $)$

\# ub3lyp/6-31g* opt freq=noraman

calculate for $1 \quad \mathrm{HF}=-482.502999$

$\begin{array}{ll}0 & 2\end{array}$

N

$\begin{array}{lrrrrrr}\mathrm{C} & 1 & 1.384174 & & & & \\ \mathrm{C} & 2 & 1.517876 & 1 & 115.153645 & & \\ \mathrm{O} & 2 & 1.231873 & 1 & 120.909651 & 3 & 174.281262 \\ \mathrm{C} & 3 & 1.530040 & 2 & 113.487547 & 1 & -175.253713 \\ \mathrm{C} & 5 & 1.539987 & 3 & 112.473732 & 2 & -178.494361 \\ \mathrm{C} & 6 & 1.504703 & 5 & 112.667708 & 3 & 178.339306 \\ \mathrm{C} & 7 & 1.333332 & 6 & 125.282076 & 5 & 119.802419 \\ \mathrm{C} & 1 & 1.451569 & 2 & 117.882808 & 4 & -34.213546 \\ \mathrm{C} & 9 & 1.542568 & 1 & 108.125143 & 2 & 103.421720 \\ \mathrm{C} & 9 & 1.538091 & 1 & 108.153080 & 2 & -135.947667 \\ \mathrm{H} & 3 & 1.099463 & 2 & 107.359498 & 1 & 61.700957 \\ \mathrm{H} & 3 & 1.099705 & 2 & 108.166111 & 1 & -51.235087 \\ \mathrm{H} & 5 & 1.096770 & 3 & 109.711482 & 2 & 59.391059 \\ \mathrm{H} & 5 & 1.096353 & 3 & 109.038261 & 2 & -56.159940 \\ \mathrm{H} & 6 & 1.098364 & 5 & 109.953393 & 3 & -59.061758 \\ \mathrm{H} & 6 & 1.101471 & 5 & 108.624333 & 3 & 57.074449 \\ \mathrm{H} & 8 & 1.086780 & 7 & 121.855021 & 6 & -179.615579\end{array}$




$\begin{array}{crrrrrr}\mathrm{H} & 8 & 1.088529 & 7 & 121.668904 & 6 & 0.578355 \\ \mathrm{H} & 7 & 1.091706 & 6 & 115.671844 & 5 & -59.468081 \\ \mathrm{H} & 9 & 1.098783 & 1 & 110.928076 & 2 & -15.233861 \\ \mathrm{H} & 10 & 1.096307 & 9 & 109.484423 & 1 & 177.449990 \\ \mathrm{H} & 10 & 1.093169 & 9 & 110.185134 & 1 & -62.997416 \\ \mathrm{H} & 10 & 1.094999 & 9 & 110.629014 & 1 & 57.502566 \\ \mathrm{H} & 11 & 1.094893 & 9 & 110.646437 & 1 & -58.810447 \\ \mathrm{H} & 11 & 1.094984 & 9 & 111.241662 & 1 & 61.632499 \\ \mathrm{H} & 11 & 1.095503 & 9 & 109.658292 & 1 & -178.748056\end{array}$

$\% \operatorname{chk}=\mathbf{1}(\mathrm{R}=t$-Bu $)$

\# ub3lyp/6-31g* opt freq=noraman

calculate for $1 \quad \mathrm{HF}=-521.815618$

$0 \quad 2$

$\mathrm{N}$

$\begin{array}{lll}\mathrm{C} & 1 & 1.380993\end{array}$

$\begin{array}{lllll}\mathrm{C} & 2 & 1.520938 & 1 & 114.623170\end{array}$

$\begin{array}{llllllr}\mathrm{O} & 2 & 1.230669 & 1 & 121.687885 & 3 & 173.626318\end{array}$

$\begin{array}{lllllll}\mathrm{C} & 3 & 1.530398 & 2 & 113.339750 & 1 & -179.276698\end{array}$

$\begin{array}{lllllll}\mathrm{C} & 5 & 1.540013 & 3 & 112.414798 & 2 & -178.491306\end{array}$

$\begin{array}{lllllll}\mathrm{C} & 6 & 1.504760 & 5 & 112.639711 & 3 & 177.985963\end{array}$

$\begin{array}{lllllll}\mathrm{C} & 7 & 1.333333 & 6 & 125.286655 & 5 & 119.835569\end{array}$

$\begin{array}{lllllll}\mathrm{C} & 1 & 2.412385 & 2 & 112.821846 & 4 & -3.927216\end{array}$

$\begin{array}{lllllll}\mathrm{C} & 1 & 1.461870 & 2 & 122.534312 & 4 & -45.947260\end{array}$

$\begin{array}{lllllll}\mathrm{C} & 10 & 1.541096 & 1 & 106.365420 & 2 & -157.369292\end{array}$

$\begin{array}{lllllll}\mathrm{C} & 10 & 1.540729 & 1 & 113.813816 & 2 & -35.452919\end{array}$

$\begin{array}{lllllll}\mathrm{H} & 3 & 1.098964 & 2 & 107.502022 & 1 & 57.581961\end{array}$

$\begin{array}{lllllll}\mathrm{H} & 3 & 1.100150 & 2 & 108.178892 & 1 & -55.568251\end{array}$

$\begin{array}{llllllr}\mathrm{H} & 5 & 1.096410 & 3 & 109.748008 & 2 & 59.309859\end{array}$

$\begin{array}{lllllll}\mathrm{H} & 5 & 1.096365 & 3 & 109.002099 & 2 & -56.231370\end{array}$

$\begin{array}{lllllll}\mathrm{H} & 6 & 1.098396 & 5 & 109.980920 & 3 & -59.426308\end{array}$

$\begin{array}{llllllr}\mathrm{H} & 6 & 1.101479 & 5 & 108.647474 & 3 & 56.732002\end{array}$

$\begin{array}{lllllll}\mathrm{H} & 8 & 1.086780 & 7 & 121.859589 & 6 & -179.626532\end{array}$

$\begin{array}{lllllll}\mathrm{H} & 8 & 1.088520 & 7 & 121.668055 & 6 & 0.568414\end{array}$

$\begin{array}{lllllll}\mathrm{H} & 7 & 1.091721 & 6 & 115.665853 & 5 & -59.433874\end{array}$

$\begin{array}{lllllll}\mathrm{H} & 9 & 1.091684 & 1 & 93.324445 & 2 & 6.942587\end{array}$

$\begin{array}{lllllll}\mathrm{H} & 9 & 1.094317 & 1 & 88.352824 & 2 & 115.762445\end{array}$

$\begin{array}{lllllll}\mathrm{H} & 9 & 1.096540 & 1 & 144.191931 & 2 & -123.798183\end{array}$

$\begin{array}{lllllllr}\mathrm{H} & 11 & 1.095520 & 10 & 110.885168 & 1 & 64.740168\end{array}$

$\begin{array}{lllllll}\mathrm{H} & 11 & 1.095652 & 10 & 110.093649 & 1 & -175.583640\end{array}$

$\begin{array}{lllllll}\mathrm{H} & 11 & 1.094273 & 10 & 110.619121 & 1 & -55.305520\end{array}$ 


$\begin{array}{lrrrrrr}\mathrm{H} & 12 & 1.095550 & 10 & 110.053328 & 1 & -179.133418 \\ \mathrm{H} & 12 & 1.096610 & 10 & 110.695834 & 1 & -60.201028 \\ \mathrm{H} & 12 & 1.092607 & 10 & 111.172513 & 1 & 60.762650\end{array}$

\%chk=1 $(\mathrm{R}=\mathrm{Ph})$

\# ub3lyp/6-31g* opt freq=noraman

calculate for $1 \quad \mathrm{HF}=-595.627557$

$0 \quad 2$

\begin{tabular}{|c|c|c|c|c|c|c|}
\hline $\mathrm{N}$ & & & & & & \\
\hline $\mathrm{C}$ & 1 & 1.393569 & & & & \\
\hline $\mathrm{C}$ & 2 & 1.522254 & 1 & 113.089213 & & \\
\hline $\mathrm{O}$ & 2 & 1.225241 & 1 & 123.541799 & 3 & 174.417457 \\
\hline $\mathrm{C}$ & 3 & 1.529418 & 2 & 113.463438 & 1 & -176.365065 \\
\hline $\mathrm{C}$ & 5 & 1.539985 & 3 & 112.499808 & 2 & -178.096798 \\
\hline $\mathrm{C}$ & 6 & 1.504647 & 5 & 112.674210 & 3 & 178.410446 \\
\hline $\mathrm{C}$ & 7 & 1.333399 & 6 & 125.333924 & 5 & 120.169123 \\
\hline $\mathrm{C}$ & 1 & 1.344408 & 2 & 122.693234 & 4 & -46.352673 \\
\hline $\mathrm{C}$ & 9 & 1.431519 & 1 & 116.682112 & 2 & 173.011857 \\
\hline $\mathrm{C}$ & 10 & 1.382657 & 9 & 120.974350 & 1 & 178.154682 \\
\hline $\mathrm{C}$ & 11 & 1.404247 & 10 & 120.008289 & 9 & -0.078938 \\
\hline $\mathrm{C}$ & 12 & 1.405160 & 11 & 120.160078 & 10 & 0.057924 \\
\hline $\mathrm{C}$ & 13 & 1.383088 & 12 & 120.710409 & 11 & -0.077793 \\
\hline $\mathrm{H}$ & 3 & 1.099375 & 2 & 107.285170 & 1 & 60.598578 \\
\hline $\mathrm{H}$ & 3 & 1.099729 & 2 & 108.272518 & 1 & -52.274031 \\
\hline $\mathrm{H}$ & 5 & 1.096682 & 3 & 109.707805 & 2 & 59.750795 \\
\hline $\mathrm{H}$ & 5 & 1.096388 & 3 & 109.026801 & 2 & -55.729693 \\
\hline $\mathrm{H}$ & 6 & 1.098439 & 5 & 109.991669 & 3 & -58.963063 \\
\hline $\mathrm{H}$ & 6 & 1.101537 & 5 & 108.628395 & 3 & 57.175570 \\
\hline $\mathrm{H}$ & 8 & 1.086836 & 7 & 121.860962 & 6 & -179.611886 \\
\hline $\mathrm{H}$ & 8 & 1.088570 & 7 & 121.670993 & 6 & 0.587221 \\
\hline $\mathrm{H}$ & 7 & 1.091770 & 6 & 115.641691 & 5 & -59.077161 \\
\hline $\mathrm{H}$ & 10 & 1.085283 & 9 & 117.293440 & 1 & -1.784326 \\
\hline $\mathrm{H}$ & 11 & 1.086365 & 10 & 120.063920 & 9 & 179.954189 \\
\hline $\mathrm{H}$ & 12 & 1.086369 & 11 & 119.971160 & 10 & -179.956350 \\
\hline $\mathrm{H}$ & 13 & 1.086585 & 12 & 119.638783 & 11 & 179.866745 \\
\hline $\mathrm{H}$ & 14 & 1.083138 & 13 & 120.790728 & 12 & 178.779416 \\
\hline
\end{tabular}

$\% \operatorname{chk}=1(\mathrm{R}=\mathrm{OMe})$

\# ub3lyp/6-31g* opt freq=noraman 
calculate for $1 \quad \mathrm{HF}=-479.054722$

$\begin{array}{lrrrrrr}0 \quad 2 & & & & \\ \mathrm{~N} & & & & & \\ \mathrm{C} & 1 & 1.419729 & & & & \\ \mathrm{C} & 2 & 1.520145 & 1 & 110.802726 & & \\ \mathrm{O} & 2 & 1.219305 & 1 & 124.629235 & 3 & 179.876466 \\ \mathrm{C} & 3 & 1.529051 & 2 & 113.083363 & 1 & -177.264530 \\ \mathrm{C} & 5 & 1.539764 & 3 & 112.457117 & 2 & -179.238984 \\ \mathrm{C} & 6 & 1.504749 & 5 & 112.703922 & 3 & 178.471812 \\ \mathrm{C} & 7 & 1.333301 & 6 & 125.267257 & 5 & 119.863700 \\ \mathrm{O} & 1 & 1.345176 & 2 & 108.709728 & 4 & 0.143642 \\ \mathrm{C} & 9 & 1.436271 & 1 & 110.341300 & 2 & -179.997589 \\ \mathrm{H} & 3 & 1.099998 & 2 & 107.972381 & 1 & 59.527017 \\ \mathrm{H} & 3 & 1.099192 & 2 & 108.198377 & 1 & -53.646357 \\ \mathrm{H} & 5 & 1.096214 & 3 & 109.526797 & 2 & 58.570851 \\ \mathrm{H} & 5 & 1.096919 & 3 & 109.199000 & 2 & -56.875278 \\ \mathrm{H} & 6 & 1.098385 & 5 & 109.903678 & 3 & -58.967784 \\ \mathrm{H} & 6 & 1.101585 & 5 & 108.687070 & 3 & 57.159685 \\ \mathrm{H} & 8 & 1.086786 & 7 & 121.869116 & 6 & -179.590504 \\ \mathrm{H} & 8 & 1.088519 & 7 & 121.645621 & 6 & 0.566402 \\ \mathrm{H} & 7 & 1.091743 & 6 & 115.685206 & 5 & -59.387245 \\ \mathrm{H} & 10 & 1.090867 & 9 & 105.373455 & 1 & 179.567156 \\ \mathrm{H} & 10 & 1.093548 & 9 & 109.954093 & 1 & -61.079742 \\ \mathrm{H} & 10 & 1.093473 & 9 & 109.962510 & 1 & 60.182183\end{array}$

Table S2. Computational (UB3LYP/6-31G*) Results on the Transition States of 6-Exo Cyclization of $\mathbf{1}$.
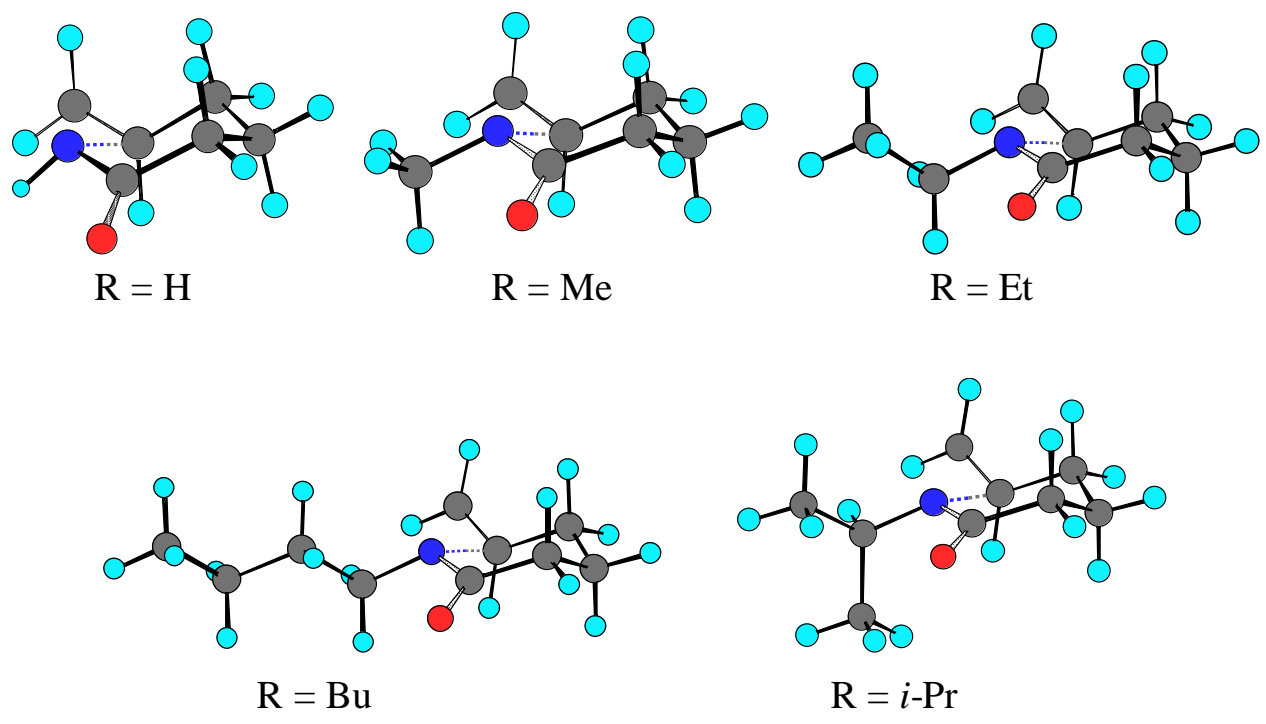


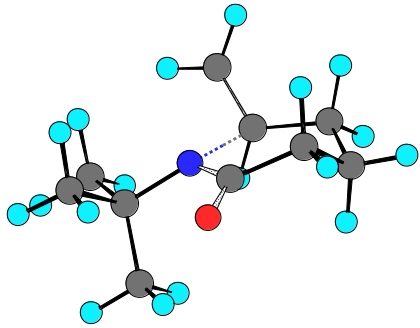

$\mathrm{R}=t-\mathrm{Bu}$

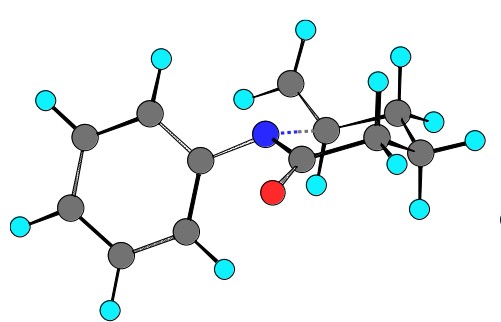

$\mathrm{R}=\mathrm{Ph}$

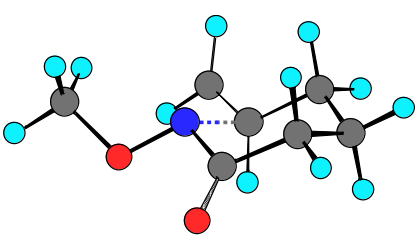

$\mathrm{R}=\mathrm{OMe}$

$\%$ chk $=\mathbf{C}-\mathbf{1}(\mathrm{R}=\mathrm{H})$

\# ub3lyp/6-31g* opt=(ts,noeigen,readfc,nofreeze) freq=noraman

calculate for $\mathrm{C}-1 \quad \mathrm{HF}=-364.544883$

$0 \quad 2$

$\mathrm{C}$

C

C

$\mathrm{N}$

C

C

C

$\mathrm{O}$

$\mathrm{H}$

$\mathrm{H}$

$\mathrm{H}$

$\mathrm{H}$

$\mathrm{H}$

$\mathrm{H}$

$\mathrm{H}$

$\mathrm{H}$

$\mathrm{H}$

$\mathrm{H}$
1.536807

$\begin{array}{lll}1.509067 & 1 & 114.637761\end{array}$

$\begin{array}{lllll}2.258553 & 2 & 96.908636 & 1 & 54.661770\end{array}$

$\begin{array}{lllll}1.373693 & 3 & 106.349693 & 2 & -52.910376\end{array}$

$\begin{array}{lllll}1.517958 & 4 & 115.344740 & 3 & 55.400676\end{array}$

$\begin{array}{llllr}1.363117 & 2 & 123.816194 & 1 & 158.411223\end{array}$

$\begin{array}{lllll}1.233921 & 4 & 121.426607 & 3 & -129.916182\end{array}$

$\begin{array}{lllll}1.097075 & 1 & 110.510743 & 6 & 56.337512\end{array}$

$\begin{array}{lllll}1.100535 & 1 & 107.982236 & 6 & 172.483403\end{array}$

$\begin{array}{lllll}1.099294 & 2 & 109.150360 & 3 & 54.963757\end{array}$

$\begin{array}{lllll}1.096571 & 2 & 108.790454 & 3 & 170.368009\end{array}$

$\begin{array}{lllll}1.097673 & 5 & 108.314036 & 4 & 58.905861\end{array}$

$\begin{array}{lllll}1.093634 & 5 & 107.889056 & 4 & 175.732324\end{array}$

$\begin{array}{lllll}1.090286 & 2 & 115.738148 & 1 & -40.328474\end{array}$

$\begin{array}{lllll}1.024911 & 3 & 101.024296 & 2 & -166.898623\end{array}$

$\begin{array}{lllll}1.086031 & 3 & 121.519990 & 2 & 170.518683\end{array}$

$\begin{array}{lllll}1.086766 & 3 & 121.336930 & 2 & -14.167198\end{array}$

$\%$ chk $=\mathbf{C}-1(\mathrm{R}=\mathrm{Me})$

\# ub3lyp/6-31g* opt=(ts,noeigen,readfc,nofreeze) freq=noraman

calculate for $\mathrm{C}-1 \quad \mathrm{HF}=-403.856293$

$0 \quad 2$

C 


$\begin{array}{lrrrrrr}\mathrm{C} & 1 & 1.533000 & & & & \\ \mathrm{C} & 2 & 1.510250 & 1 & 114.348255 & & \\ \mathrm{~N} & 3 & 2.203512 & 2 & 98.957697 & 1 & 53.546396 \\ \mathrm{C} & 4 & 1.375388 & 3 & 108.369324 & 2 & -49.162637 \\ \mathrm{C} & 5 & 1.520476 & 4 & 116.055446 & 3 & 50.160228 \\ \mathrm{C} & 3 & 1.370006 & 2 & 122.350028 & 1 & 164.044234 \\ \mathrm{O} & 5 & 1.233898 & 4 & 121.672246 & 3 & -136.553365 \\ \mathrm{C} & 4 & 1.452633 & 3 & 107.906507 & 2 & -175.574438 \\ \mathrm{H} & 2 & 1.097328 & 1 & 110.474594 & 6 & 56.807986 \\ \mathrm{H} & 2 & 1.100324 & 1 & 108.370740 & 6 & 173.091412 \\ \mathrm{H} & 1 & 1.099360 & 2 & 109.241781 & 3 & 55.536985 \\ \mathrm{H} & 1 & 1.096745 & 2 & 109.040087 & 3 & 171.168667 \\ \mathrm{H} & 6 & 1.098321 & 5 & 108.041439 & 4 & 63.232012 \\ \mathrm{H} & 6 & 1.093689 & 5 & 107.099434 & 4 & 179.057920 \\ \mathrm{H} & 3 & 1.090172 & 2 & 115.867661 & 1 & -40.056686 \\ \mathrm{H} & 7 & 1.085409 & 3 & 121.726076 & 2 & 167.996726 \\ \mathrm{H} & 7 & 1.087101 & 3 & 121.195071 & 2 & -16.410769 \\ \mathrm{H} & 9 & 1.099265 & 4 & 113.815707 & 3 & 70.217862 \\ \mathrm{H} & 9 & 1.096720 & 4 & 108.605024 & 3 & -170.765331 \\ \mathrm{H} & 9 & 1.092427 & 4 & 109.033570 & 3 & -53.017710 \\ & & & & & & \end{array}$

\%chk $=\mathbf{C}-1 \quad(\mathrm{R}=\mathrm{Et})$

\# ub3lyp/6-31g* opt=(ts,noeigen,readfc,nofreeze) freq=noraman

calculate for $\mathrm{C}-1 \quad \mathrm{HF}=-443.172035$

$\begin{array}{llllllr}0 \quad 2 & & & & \\ \mathrm{C} & & & & & & \\ \mathrm{C} & 1 & 1.532008 & & & & \\ \mathrm{C} & 2 & 1.509587 & 1 & 114.955322 & & \\ \mathrm{~N} & 3 & 2.193480 & 2 & 100.929825 & 1 & 48.298955 \\ \mathrm{C} & 4 & 1.368518 & 3 & 111.287870 & 2 & -39.623099 \\ \mathrm{C} & 5 & 1.519397 & 4 & 117.196706 & 3 & 42.059399 \\ \mathrm{C} & 3 & 1.371867 & 2 & 121.583425 & 1 & 159.736872 \\ \mathrm{O} & 5 & 1.236546 & 4 & 121.072751 & 3 & -144.226300 \\ \mathrm{C} & 4 & 1.460817 & 3 & 109.506867 & 2 & -170.718568 \\ \mathrm{C} & 9 & 1.535299 & 4 & 111.174296 & 3 & -143.958914 \\ \mathrm{H} & 2 & 1.097740 & 1 & 110.162620 & 6 & 56.968262 \\ \mathrm{H} & 2 & 1.100489 & 1 & 108.475983 & 6 & 172.995762 \\ \mathrm{H} & 1 & 1.099195 & 2 & 109.507892 & 3 & 55.728706 \\ \mathrm{H} & 1 & 1.096817 & 2 & 108.910868 & 3 & 171.483432 \\ \mathrm{H} & 6 & 1.098628 & 5 & 108.247174 & 4 & 66.346493 \\ \mathrm{H} & 6 & 1.093808 & 5 & 106.769279 & 4 & -178.107974\end{array}$




$\begin{array}{lrrrrrr}\mathrm{H} & 3 & 1.089642 & 2 & 115.933271 & 1 & -45.353603 \\ \mathrm{H} & 7 & 1.085359 & 3 & 121.928569 & 2 & 167.981826 \\ \mathrm{H} & 7 & 1.086974 & 3 & 121.051385 & 2 & -16.837281 \\ \mathrm{H} & 9 & 1.098178 & 4 & 111.202656 & 3 & 93.612263 \\ \mathrm{H} & 9 & 1.094161 & 4 & 107.523284 & 3 & -24.925183 \\ \mathrm{H} & 10 & 1.092656 & 9 & 110.207618 & 4 & -64.574557 \\ \mathrm{H} & 10 & 1.095093 & 9 & 110.680753 & 4 & 55.788137 \\ \mathrm{H} & 10 & 1.096911 & 9 & 109.972848 & 4 & 175.690623\end{array}$

$\% \operatorname{chk}=\mathbf{C}-\mathbf{1}(\mathrm{R}=\mathrm{Bu})$

\# ub3lyp/6-31g* opt=(ts,noeigen,readfc,nofreeze) freq=noraman

calculate for $\mathrm{C}-1 \quad \mathrm{HF}=-521.799680$

\begin{tabular}{|c|c|c|c|c|c|c|}
\hline & & & & & & \\
\hline C & 1 & 1.531894 & & & & \\
\hline $\mathrm{C}$ & 2 & 1.509684 & 1 & 114.921199 & & \\
\hline $\mathrm{N}$ & 3 & 2.188166 & 2 & 101.075568 & 1 & 48.519959 \\
\hline $\mathrm{C}$ & 4 & 1.369582 & 3 & 110.915325 & 2 & -40.427163 \\
\hline $\mathrm{C}$ & 5 & 1.519643 & 4 & 117.102297 & 3 & 43.008729 \\
\hline $\mathrm{C}$ & 3 & 1.372531 & 2 & 121.544839 & 1 & 160.380080 \\
\hline $\mathrm{O}$ & 5 & 1.236384 & 4 & 121.232881 & 3 & -143.215965 \\
\hline $\mathrm{C}$ & 4 & 1.459548 & 3 & 109.361789 & 2 & -170.886871 \\
\hline $\mathrm{C}$ & 9 & 1.539575 & 4 & 111.470260 & 3 & -145.576810 \\
\hline $\mathrm{C}$ & 10 & 1.534139 & 9 & 112.286192 & 4 & 176.469335 \\
\hline $\mathrm{C}$ & 11 & 1.532388 & 10 & 112.802684 & 9 & 179.482076 \\
\hline $\mathrm{H}$ & 2 & 1.097723 & 1 & 110.154401 & 6 & 57.335284 \\
\hline $\mathrm{H}$ & 2 & 1.100416 & 1 & 108.504181 & 6 & 173.393624 \\
\hline $\mathrm{H}$ & 1 & 1.099274 & 2 & 109.489148 & 3 & 56.074833 \\
\hline $\mathrm{H}$ & 1 & 1.096835 & 2 & 108.952709 & 3 & 171.837941 \\
\hline $\mathrm{H}$ & 6 & 1.098553 & 5 & 108.272874 & 4 & 65.760677 \\
\hline $\mathrm{H}$ & 6 & 1.093786 & 5 & 106.776485 & 4 & -178.659391 \\
\hline $\mathrm{H}$ & 3 & 1.089618 & 2 & 115.897957 & 1 & -45.141642 \\
\hline $\mathrm{H}$ & 7 & 1.085342 & 3 & 121.905601 & 2 & 167.788868 \\
\hline $\mathrm{H}$ & 7 & 1.087028 & 3 & 121.096225 & 2 & -17.041683 \\
\hline $\mathrm{H}$ & 9 & 1.099030 & 4 & 111.318973 & 3 & 91.974977 \\
\hline $\mathrm{H}$ & 9 & 1.094901 & 4 & 107.678244 & 3 & -26.690273 \\
\hline $\mathrm{H}$ & 10 & 1.095362 & 9 & 108.434892 & 4 & -61.799681 \\
\hline $\mathrm{H}$ & 10 & 1.098213 & 9 & 108.801225 & 4 & 54.432051 \\
\hline $\mathrm{H}$ & 11 & 1.099933 & 10 & 109.542765 & 9 & -58.382467 \\
\hline $\mathrm{H}$ & 11 & 1.098983 & 10 & 109.319064 & 9 & 57.564897 \\
\hline $\mathrm{H}$ & 12 & 1.096982 & 11 & 111.179671 & 10 & 59.957346 \\
\hline
\end{tabular}




$\begin{array}{llllllr}\mathrm{H} & 12 & 1.096162 & 11 & 111.394254 & 10 & -179.872533 \\ \mathrm{H} & 12 & 1.096608 & 11 & 111.066231 & 10 & -59.752979\end{array}$

$\%$ chk $=\mathbf{C}-\mathbf{1}(\mathrm{R}=i$-Pr $)$

\# ub3lyp/6-31g* opt=(ts,noeigen,readfc,nofreeze $)$ freq=noraman

calculate for $\mathrm{C}-1 \quad \mathrm{HF}=-482.485402$

\begin{tabular}{|c|c|c|c|c|c|c|}
\hline $\mathrm{C}$ & 1 & 1.531511 & & & & \\
\hline $\mathrm{C}$ & 2 & 1.509078 & 1 & 114.976012 & & \\
\hline $\mathrm{N}$ & 3 & 2.197440 & 2 & 101.467663 & 1 & 45.336872 \\
\hline $\mathrm{C}$ & 4 & 1.360099 & 3 & 113.830546 & 2 & -32.800556 \\
\hline $\mathrm{C}$ & 5 & 1.520688 & 4 & 117.569720 & 3 & 34.535454 \\
\hline $\mathrm{C}$ & 3 & 1.372278 & 2 & 121.572375 & 1 & 157.529909 \\
\hline $\mathrm{O}$ & 5 & 1.239238 & 4 & 121.597735 & 3 & -152.193940 \\
\hline $\mathrm{C}$ & 4 & 1.467344 & 3 & 109.433452 & 2 & -172.846458 \\
\hline $\mathrm{C}$ & 9 & 1.542023 & 4 & 110.211868 & 3 & -150.054566 \\
\hline $\mathrm{C}$ & 9 & 1.536951 & 4 & 114.511664 & 3 & 82.587351 \\
\hline $\mathrm{H}$ & 2 & 1.097862 & 1 & 110.095167 & 6 & 56.340542 \\
\hline $\mathrm{H}$ & 2 & 1.100543 & 1 & 108.558489 & 6 & 172.351000 \\
\hline $\mathrm{H}$ & 1 & 1.099167 & 2 & 109.574133 & 3 & 55.116382 \\
\hline $\mathrm{H}$ & 1 & 1.096975 & 2 & 108.857281 & 3 & 170.853407 \\
\hline $\mathrm{H}$ & 6 & 1.098963 & 5 & 108.204947 & 4 & 71.162044 \\
\hline $\mathrm{H}$ & 6 & 1.094041 & 5 & 106.319497 & 4 & -173.907608 \\
\hline $\mathrm{H}$ & 3 & 1.089088 & 2 & 115.758293 & 1 & -48.098715 \\
\hline $\mathrm{H}$ & 7 & 1.085336 & 3 & 121.899739 & 2 & 167.423604 \\
\hline $\mathrm{H}$ & 7 & 1.087060 & 3 & 121.120855 & 2 & -17.088533 \\
\hline $\mathrm{H}$ & 9 & 1.095582 & 4 & 104.809720 & 3 & -35.857734 \\
\hline $\mathrm{H}$ & 10 & 1.091716 & 9 & 110.493878 & 4 & -69.476679 \\
\hline $\mathrm{H}$ & 10 & 1.094814 & 9 & 110.418917 & 4 & 50.818452 \\
\hline $\mathrm{H}$ & 10 & 1.096955 & 9 & 109.603445 & 4 & 170.133444 \\
\hline $\mathrm{H}$ & 11 & 1.096557 & 9 & 110.109989 & 4 & -175.746653 \\
\hline $\mathrm{H}$ & 11 & 1.096803 & 9 & 111.188434 & 4 & -56.522848 \\
\hline $\mathrm{H}$ & 11 & 1.092185 & 9 & 110.594475 & 4 & 64.075542 \\
\hline
\end{tabular}

$\%$ chk $=\mathbf{C}-1(\mathrm{R}=t-\mathrm{Bu})$

\# ub3lyp/6-31g* opt=(ts,noeigen,readfc,nofreeze) freq=noraman

calculate for $\mathrm{C}-1 \quad \mathrm{HF}=-521.791658$ 


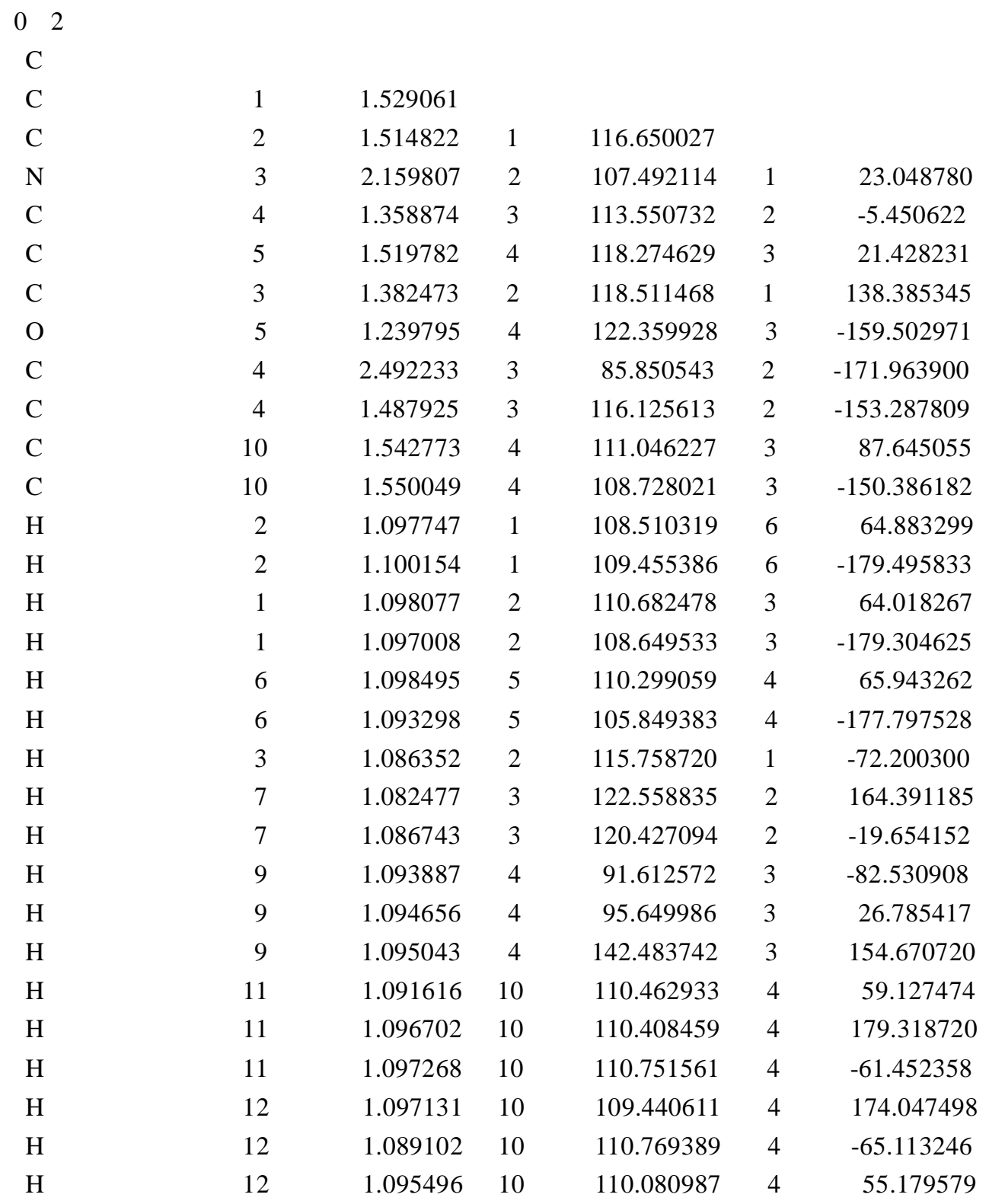

\%chk=C-1 $(\mathrm{R}=\mathrm{Ph})$

\# ub3lyp/6-31g* opt=(ts,noeigen,readfc,nofreeze) freq=noraman

calculate for $\mathrm{C}-1 \quad \mathrm{HF}=-595.599044$

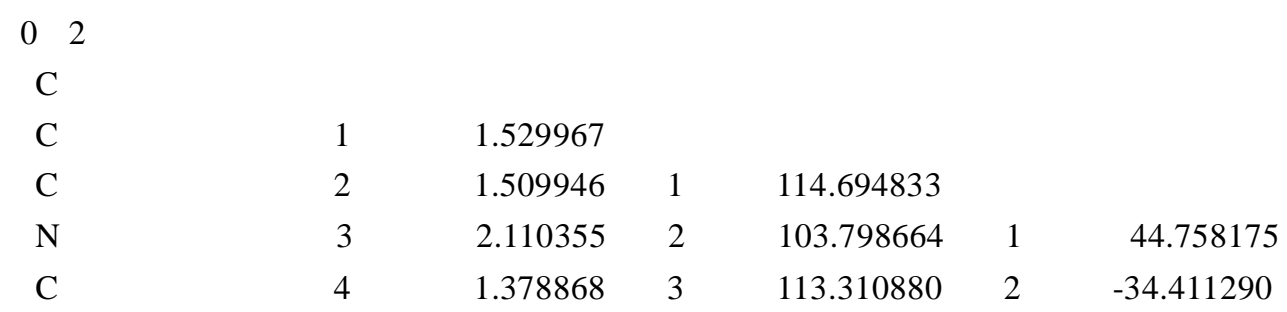




\begin{tabular}{|c|c|c|c|c|c|c|}
\hline $\mathrm{C}$ & 5 & 1.521034 & 4 & 116.549951 & 3 & 37.661293 \\
\hline $\mathrm{C}$ & 3 & 1.384681 & 2 & 120.390944 & 1 & 160.101996 \\
\hline $\mathrm{O}$ & 5 & 1.230124 & 4 & 122.222882 & 3 & -149.079415 \\
\hline $\mathrm{C}$ & 4 & 1.404370 & 3 & 108.118290 & 2 & -171.048928 \\
\hline $\mathrm{C}$ & 9 & 1.405941 & 4 & 118.047105 & 3 & -88.405845 \\
\hline $\mathrm{C}$ & 10 & 1.392990 & 9 & 120.678649 & 4 & 177.532696 \\
\hline $\mathrm{C}$ & 11 & 1.397455 & 10 & 120.104057 & 9 & -0.118181 \\
\hline $\mathrm{C}$ & 12 & 1.398415 & 11 & 119.690323 & 10 & 0.089568 \\
\hline $\mathrm{C}$ & 13 & 1.391713 & 12 & 120.430089 & 11 & -0.295409 \\
\hline $\mathrm{H}$ & 2 & 1.097929 & 1 & 109.994240 & 6 & 58.754218 \\
\hline $\mathrm{H}$ & 2 & 1.100346 & 1 & 108.933258 & 6 & 174.983440 \\
\hline $\mathrm{H}$ & 1 & 1.099248 & 2 & 109.696058 & 3 & 57.604670 \\
\hline $\mathrm{H}$ & 1 & 1.096793 & 2 & 109.093019 & 3 & 173.640404 \\
\hline $\mathrm{H}$ & 6 & 1.098932 & 5 & 108.242423 & 4 & 68.026924 \\
\hline $\mathrm{H}$ & 6 & 1.093462 & 5 & 106.351518 & 4 & -176.766568 \\
\hline $\mathrm{H}$ & 3 & 1.088968 & 2 & 115.642000 & 1 & -50.252171 \\
\hline $\mathrm{H}$ & 7 & 1.084500 & 3 & 121.551872 & 2 & 168.146926 \\
\hline $\mathrm{H}$ & 7 & 1.087026 & 3 & 120.921166 & 2 & -18.577298 \\
\hline $\mathrm{H}$ & 10 & 1.085874 & 9 & 118.374739 & 4 & -3.089984 \\
\hline $\mathrm{H}$ & 11 & 1.086896 & 10 & 119.795306 & 9 & 179.588157 \\
\hline $\mathrm{H}$ & 12 & 1.086658 & 11 & 120.175862 & 10 & 179.918400 \\
\hline $\mathrm{H}$ & 13 & 1.086913 & 12 & 119.967514 & 11 & 179.607889 \\
\hline $\mathrm{H}$ & 14 & 1.084548 & 13 & 120.384310 & 12 & 178.761471 \\
\hline
\end{tabular}

$\%$ chk $=\mathbf{C}-\mathbf{1}(\mathrm{R}=\mathrm{OMe})$

\# ub3lyp/6-31g* opt=(ts,noeigen,readfc,nofreeze) freq=noraman

calculate for $\mathrm{C}-1 \quad \mathrm{HF}=-479.026368$

\begin{tabular}{|c|c|c|c|c|c|c|}
\hline $\mathrm{C}$ & 1 & 1.530895 & & & & \\
\hline $\mathrm{C}$ & 2 & 1.516651 & 1 & 113.391135 & & \\
\hline $\mathrm{N}$ & 3 & 2.018947 & 2 & 100.673499 & 1 & 56.820638 \\
\hline $\mathrm{C}$ & 4 & 1.412602 & 3 & 105.381913 & 2 & -59.572225 \\
\hline $\mathrm{C}$ & 5 & 1.523201 & 4 & 111.822299 & 3 & 61.658673 \\
\hline $\mathrm{C}$ & 3 & 1.392856 & 2 & 120.100981 & 1 & 173.583588 \\
\hline $\mathrm{O}$ & 5 & 1.218723 & 4 & 124.572829 & 3 & -123.032600 \\
\hline $\mathrm{O}$ & 4 & 1.383630 & 3 & 105.954386 & 2 & -175.535556 \\
\hline $\mathrm{C}$ & 9 & 1.423307 & 4 & 109.338824 & 3 & -98.897189 \\
\hline $\mathrm{H}$ & 2 & 1.097700 & 1 & 110.487292 & 6 & 61.146112 \\
\hline $\mathrm{H}$ & 2 & 1.099048 & 1 & 109.056686 & 6 & 177.975987 \\
\hline $\mathrm{H}$ & 1 & 1.098784 & 2 & 109.330643 & 3 & 59.943199 \\
\hline
\end{tabular}




$\begin{array}{lcccccc}\mathrm{H} & 1 & 1.096564 & 2 & 109.690275 & 3 & 176.280979 \\ \mathrm{H} & 6 & 1.097567 & 5 & 108.619472 & 4 & 57.120327 \\ \mathrm{H} & 6 & 1.093178 & 5 & 106.953972 & 4 & 173.612858 \\ \mathrm{H} & 3 & 1.090256 & 2 & 115.442345 & 1 & -40.585303 \\ \mathrm{H} & 7 & 1.084958 & 3 & 121.327546 & 2 & 164.005580 \\ \mathrm{H} & 7 & 1.087079 & 3 & 121.158559 & 2 & -20.747924 \\ \mathrm{H} & 10 & 1.098065 & 9 & 111.008386 & 4 & 62.128875 \\ \mathrm{H} & 10 & 1.092875 & 9 & 105.460594 & 4 & -178.802798 \\ \mathrm{H} & 10 & 1.095494 & 9 & 110.829903 & 4 & -59.477710\end{array}$

Table S3. Computational (UB3LYP/6-31G*) Results on the Transition States of 1,5-H Abstraction of $\mathbf{1}$.
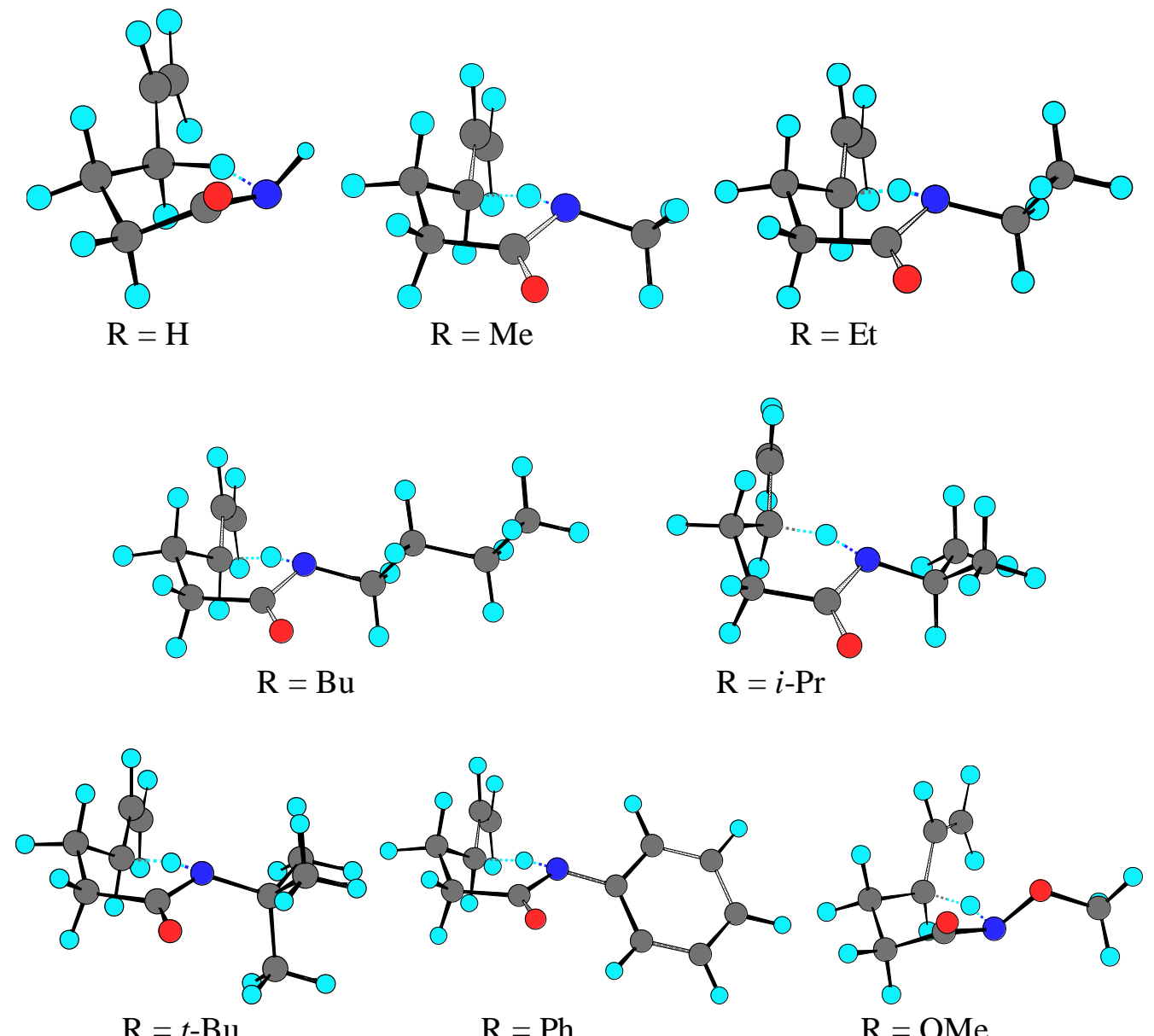

\%chk=M-1 $(\mathrm{R}=\mathrm{H})$

\# ub3lyp/6-31g* opt=(ts,noeigen,readfc,nofreeze) freq=noraman

calculate for M-1 $\mathrm{HF}=-364.539035$ 


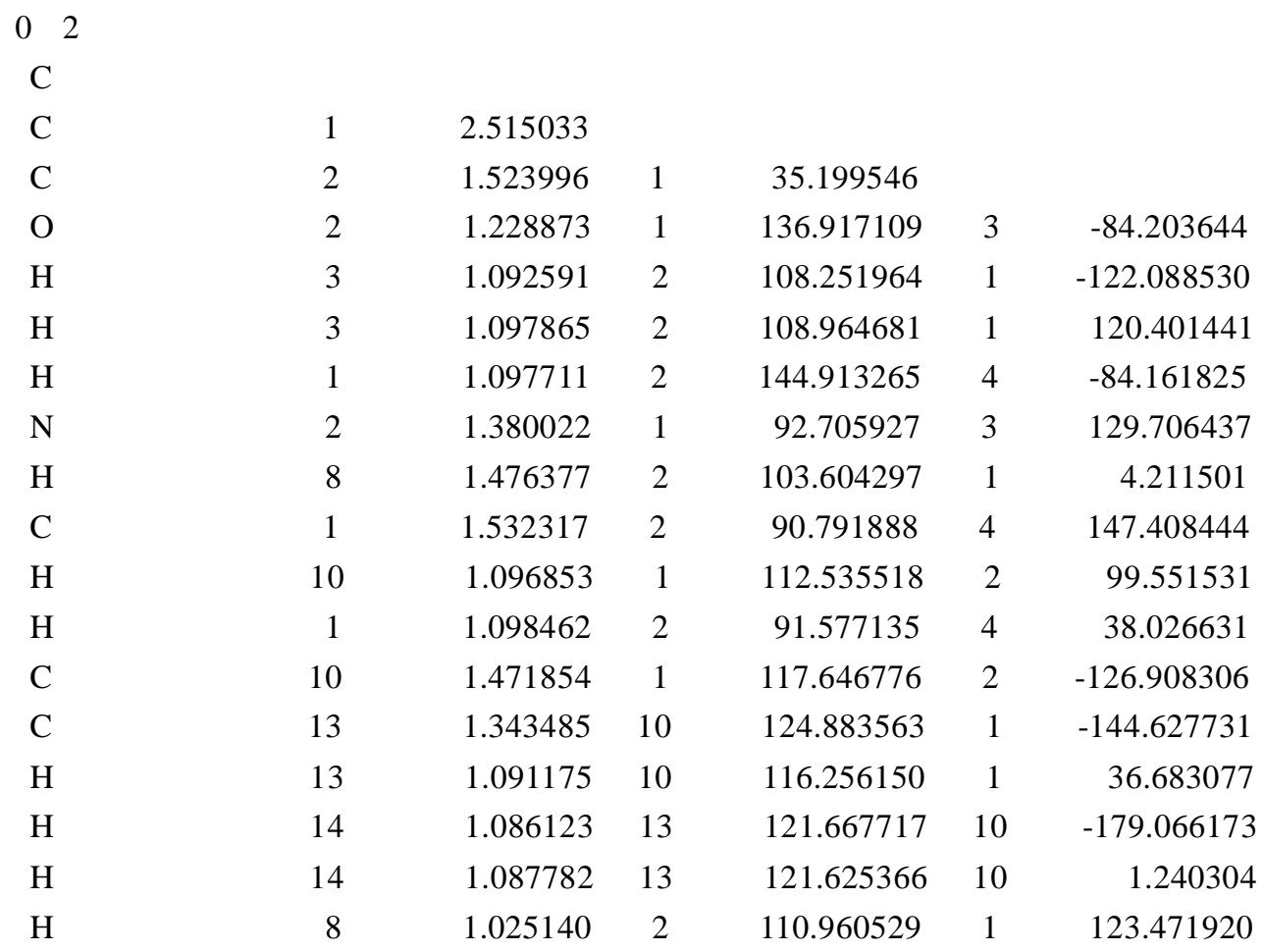

$\%$ chk=M-1 $(\mathrm{R}=\mathrm{Me})$

\# ub3lyp/6-31g* opt=(ts,noeigen,readfc,nofreeze $)$ freq=noraman

calculate for $\mathrm{M}-1 \quad \mathrm{HF}=-403.853145$

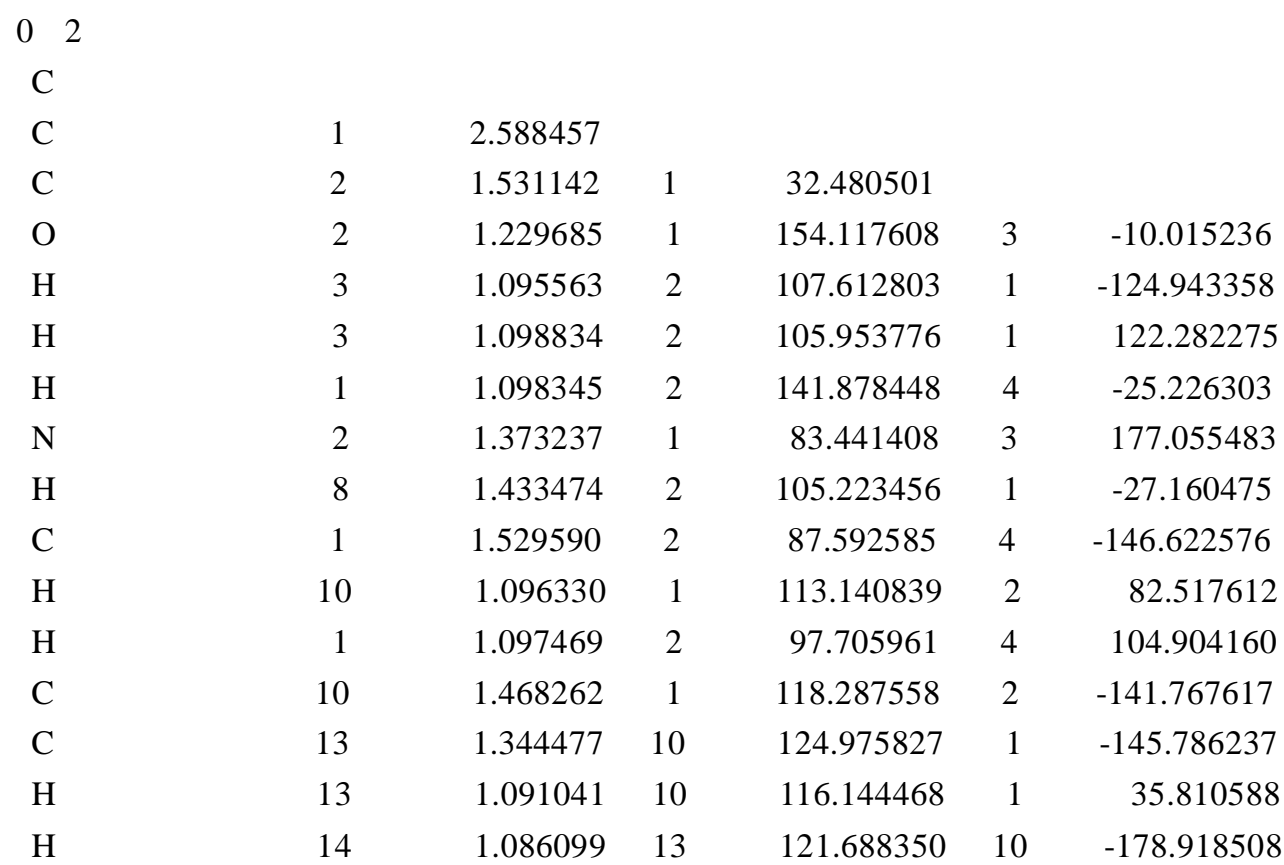




$\begin{array}{ccccccc}\mathrm{H} & 14 & 1.087872 & 13 & 121.618988 & 10 & 1.472740 \\ \mathrm{C} & 8 & 1.450629 & 2 & 118.401244 & 1 & -159.171113 \\ \mathrm{H} & 18 & 1.098046 & 8 & 108.968711 & 2 & -85.298487 \\ \mathrm{H} & 18 & 1.096851 & 8 & 113.107161 & 2 & 34.707971 \\ \mathrm{H} & 18 & 1.094608 & 8 & 108.898636 & 2 & 157.708157\end{array}$

$\%$ chk=M-1 $(\mathrm{R}=\mathrm{Et})$

\# ub3lyp/6-31g* opt=(ts,noeigen,readfc,nofreeze) freq=noraman

calculate for $\mathrm{M}-1 \quad \mathrm{HF}=-443.170030$

\begin{tabular}{|c|c|c|c|c|c|c|}
\hline $\mathrm{C}$ & 1 & 2.587621 & & & & \\
\hline $\mathrm{C}$ & 2 & 1.531053 & 1 & 32.500328 & & \\
\hline $\mathrm{O}$ & 2 & 1.230895 & 1 & 153.758191 & 3 & -13.951125 \\
\hline $\mathrm{N}$ & 2 & 1.371984 & 1 & 83.643430 & 3 & 175.205709 \\
\hline $\mathrm{C}$ & 1 & 1.529370 & 2 & 87.855437 & 4 & -149.884489 \\
\hline $\mathrm{C}$ & 6 & 1.468275 & 1 & 118.283771 & 2 & -141.123386 \\
\hline $\mathrm{C}$ & 7 & 1.344481 & 6 & 125.008953 & 1 & -145.885833 \\
\hline $\mathrm{C}$ & 5 & 1.457083 & 2 & 119.027076 & 1 & -159.521952 \\
\hline $\mathrm{C}$ & 9 & 1.534904 & 5 & 110.998847 & 2 & -95.207165 \\
\hline $\mathrm{H}$ & 3 & 1.095384 & 2 & 107.575865 & 1 & -124.967370 \\
\hline $\mathrm{H}$ & 3 & 1.099059 & 2 & 105.993833 & 1 & 122.221586 \\
\hline $\mathrm{H}$ & 1 & 1.098381 & 2 & 142.008421 & 4 & -27.984286 \\
\hline $\mathrm{H}$ & 6 & 1.238124 & 1 & 96.708823 & 2 & -26.235651 \\
\hline $\mathrm{H}$ & 6 & 1.096337 & 1 & 113.145210 & 2 & 83.188263 \\
\hline $\mathrm{H}$ & 1 & 1.097529 & 2 & 97.311590 & 4 & 101.571707 \\
\hline $\mathrm{H}$ & 7 & 1.091055 & 6 & 116.127545 & 1 & 35.774813 \\
\hline $\mathrm{H}$ & 8 & 1.086104 & 7 & 121.682690 & 6 & -178.847485 \\
\hline $\mathrm{H}$ & 8 & 1.087880 & 7 & 121.631391 & 6 & 1.526703 \\
\hline $\mathrm{H}$ & 9 & 1.097540 & 5 & 110.761581 & 2 & 27.120643 \\
\hline $\mathrm{H}$ & 9 & 1.097438 & 5 & 107.243564 & 2 & 145.322456 \\
\hline $\mathrm{H}$ & 10 & 1.096477 & 9 & 109.954192 & 5 & -176.721672 \\
\hline $\mathrm{H}$ & 10 & 1.094768 & 9 & 110.836397 & 5 & -56.579392 \\
\hline $\mathrm{H}$ & 10 & 1.093151 & 9 & 109.898722 & 5 & 63.660238 \\
\hline
\end{tabular}

$\% \operatorname{chk}=\mathrm{M}-1(\mathrm{R}=\mathrm{Bu})$

\# ub3lyp/6-31 $\mathrm{g} * \mathrm{opt}=(\mathrm{ts}$, noeigen,readfc,nofreeze $)$ freq=noraman

calculate for $\mathrm{M}-1 \quad \mathrm{HF}=-521.797833$ 


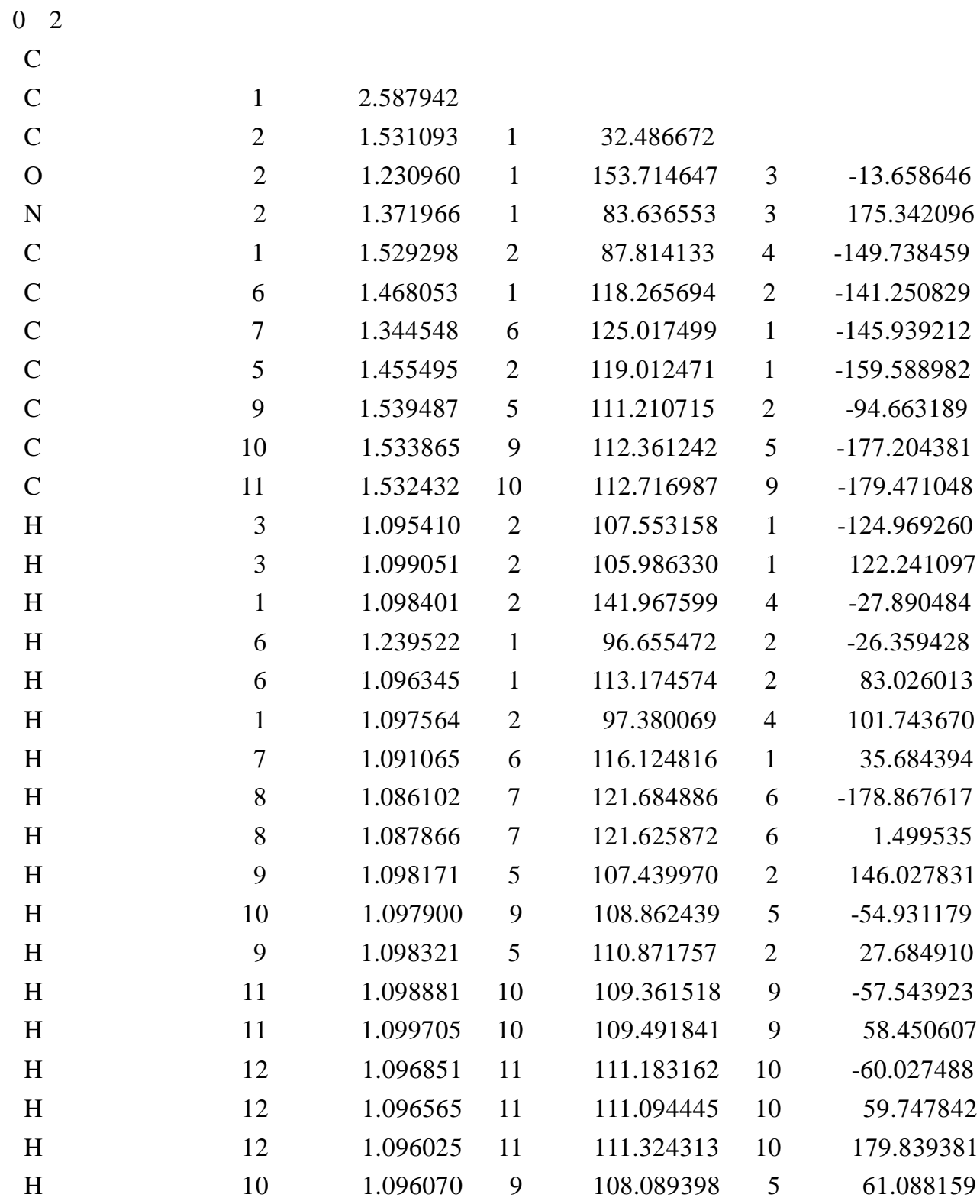

\%chk=M-1 $(\mathrm{R}=i$-Pr $)$

\# ub3lyp/6-31g* opt=(ts,noeigen,readfc,nofreeze) freq=noraman

calculate for $\mathrm{M}-1 \quad \mathrm{HF}=-482.485912$

$\begin{array}{lllllll}0 \quad 2 & & & & & \\ \mathrm{C} & 1 & 2.588683 & & & & \\ \mathrm{C} & 2 & 1.531873 & 1 & 32.445938 & & \\ \mathrm{C} & 2 & 1.231416 & 1 & 153.315269 & 3 & -14.070541 \\ \mathrm{O} & 2 & 1.371986 & 1 & 83.759609 & 3 & 175.152577 \\ \mathrm{~N} & & & & & & \end{array}$




$\begin{array}{lrrrrrr}\mathrm{C} & 1 & 1.529141 & 2 & 87.856622 & 4 & -149.743789 \\ \mathrm{C} & 6 & 1.468016 & 1 & 118.320853 & 2 & -140.968113 \\ \mathrm{C} & 7 & 1.344463 & 6 & 124.986560 & 1 & -146.068727 \\ \mathrm{C} & 5 & 1.464333 & 2 & 119.193306 & 1 & -160.471030 \\ \mathrm{C} & 9 & 1.539381 & 5 & 108.700673 & 2 & -89.325393 \\ \mathrm{C} & 9 & 1.532945 & 5 & 109.664082 & 2 & 148.713329 \\ \mathrm{H} & 3 & 1.095356 & 2 & 107.456087 & 1 & -124.954861 \\ \mathrm{H} & 3 & 1.099026 & 2 & 106.028231 & 1 & 122.266424 \\ \mathrm{H} & 1 & 1.098453 & 2 & 142.055616 & 4 & -27.777257 \\ \mathrm{H} & 6 & 1.240698 & 1 & 96.646134 & 2 & -26.072039 \\ \mathrm{H} & 6 & 1.096369 & 1 & 113.139115 & 2 & 83.305405 \\ \mathrm{H} & 1 & 1.097561 & 2 & 97.202670 & 4 & 101.691404 \\ \mathrm{H} & 7 & 1.091042 & 6 & 116.135834 & 1 & 35.596539 \\ \mathrm{H} & 8 & 1.086111 & 7 & 121.705303 & 6 & -178.841652 \\ \mathrm{H} & 8 & 1.087889 & 7 & 121.620409 & 6 & 1.574850 \\ \mathrm{H} & 9 & 1.099065 & 5 & 109.336639 & 2 & 28.473201 \\ \mathrm{H} & 10 & 1.096884 & 9 & 109.822052 & 5 & -179.837249 \\ \mathrm{H} & 10 & 1.095682 & 9 & 110.543955 & 5 & -60.000123 \\ \mathrm{H} & 10 & 1.092356 & 9 & 110.053478 & 5 & 60.426187 \\ \mathrm{H} & 11 & 1.095170 & 9 & 109.952568 & 5 & 178.305761 \\ \mathrm{H} & 11 & 1.095640 & 9 & 111.487647 & 5 & -61.897050 \\ \mathrm{H} & 11 & 1.095988 & 9 & 110.834292 & 5 & 58.652997 \\ & & & & & & \end{array}$

$\% \operatorname{chk}=\mathrm{M}-1(\mathrm{R}=t-\mathrm{Bu})$

\# ub3lyp/6-31g* opt=(ts,noeigen,readfc,nofreeze) freq=noraman

calculate for $\mathrm{M}-1 \quad \mathrm{HF}=-521.799381$

\begin{tabular}{|c|c|c|c|c|c|c|}
\hline $\mathrm{C}$ & 1 & 2.594749 & & & & \\
\hline $\mathrm{C}$ & 1 & 1.533788 & 2 & 32.258218 & & \\
\hline $\mathrm{O}$ & 2 & 1.232589 & 1 & 151.618929 & 3 & -21.380110 \\
\hline $\mathrm{N}$ & 2 & 1.369684 & 1 & 83.873289 & 3 & 171.827261 \\
\hline $\mathrm{C}$ & 1 & 1.528348 & 2 & 88.407286 & 4 & -155.517482 \\
\hline $\mathrm{C}$ & 6 & 1.469131 & 1 & 118.333938 & 2 & -140.113837 \\
\hline $\mathrm{C}$ & 7 & 1.344085 & 6 & 124.972127 & 1 & -145.160003 \\
\hline $\mathrm{C}$ & 5 & 2.420465 & 2 & 159.914890 & 1 & -141.676065 \\
\hline $\mathrm{C}$ & 5 & 1.473764 & 2 & 123.872857 & 1 & -160.327221 \\
\hline $\mathrm{C}$ & 10 & 1.548286 & 5 & 107.689730 & 2 & -73.289762 \\
\hline $\mathrm{C}$ & 10 & 1.540110 & 5 & 112.210713 & 2 & 48.523317 \\
\hline $\mathrm{H}$ & 3 & 1.095049 & 1 & 111.685507 & 2 & 122.665853 \\
\hline $\mathrm{H}$ & 3 & 1.099439 & 1 & 110.131686 & 2 & -120.024371 \\
\hline
\end{tabular}




$\begin{array}{rrrrrrr}\mathrm{H} & 1 & 1.098527 & 2 & 142.155127 & 4 & -32.621175 \\ \mathrm{H} & 5 & 1.441319 & 2 & 105.576684 & 1 & -25.343822 \\ \mathrm{H} & 6 & 1.096422 & 1 & 113.018288 & 2 & 84.447477 \\ \mathrm{H} & 1 & 1.097532 & 2 & 96.467405 & 4 & 95.764822 \\ \mathrm{H} & 7 & 1.091051 & 6 & 116.132328 & 1 & 36.369133 \\ \mathrm{H} & 8 & 1.086132 & 7 & 121.703232 & 6 & -178.942503 \\ \mathrm{H} & 8 & 1.087932 & 7 & 121.627605 & 6 & 1.541655 \\ \mathrm{H} & 9 & 1.096082 & 5 & 93.079429 & 2 & 97.491298 \\ \mathrm{H} & 9 & 1.095157 & 5 & 89.376640 & 2 & -154.358827 \\ \mathrm{H} & 9 & 1.095277 & 5 & 145.638269 & 2 & -31.311914 \\ \mathrm{H} & 11 & 1.090986 & 10 & 110.453622 & 5 & 64.561346 \\ \mathrm{H} & 11 & 1.096896 & 10 & 109.294227 & 5 & -174.988343 \\ \mathrm{H} & 11 & 1.094950 & 10 & 110.399059 & 5 & -55.766157 \\ \mathrm{H} & 12 & 1.096155 & 10 & 110.305884 & 5 & -179.723688 \\ \mathrm{H} & 12 & 1.092601 & 10 & 110.692003 & 5 & -59.600674 \\ \mathrm{H} & 12 & 1.096792 & 10 & 110.654278 & 5 & 61.063992\end{array}$

$\%$ chk=M-1 $(\mathrm{R}=\mathrm{Ph})$

\# ub3lyp/6-31g* opt=(ts,noeigen,readfc,nofreeze) freq=noraman

calculate for $\mathrm{M}-1 \quad \mathrm{HF}=-595.600241$

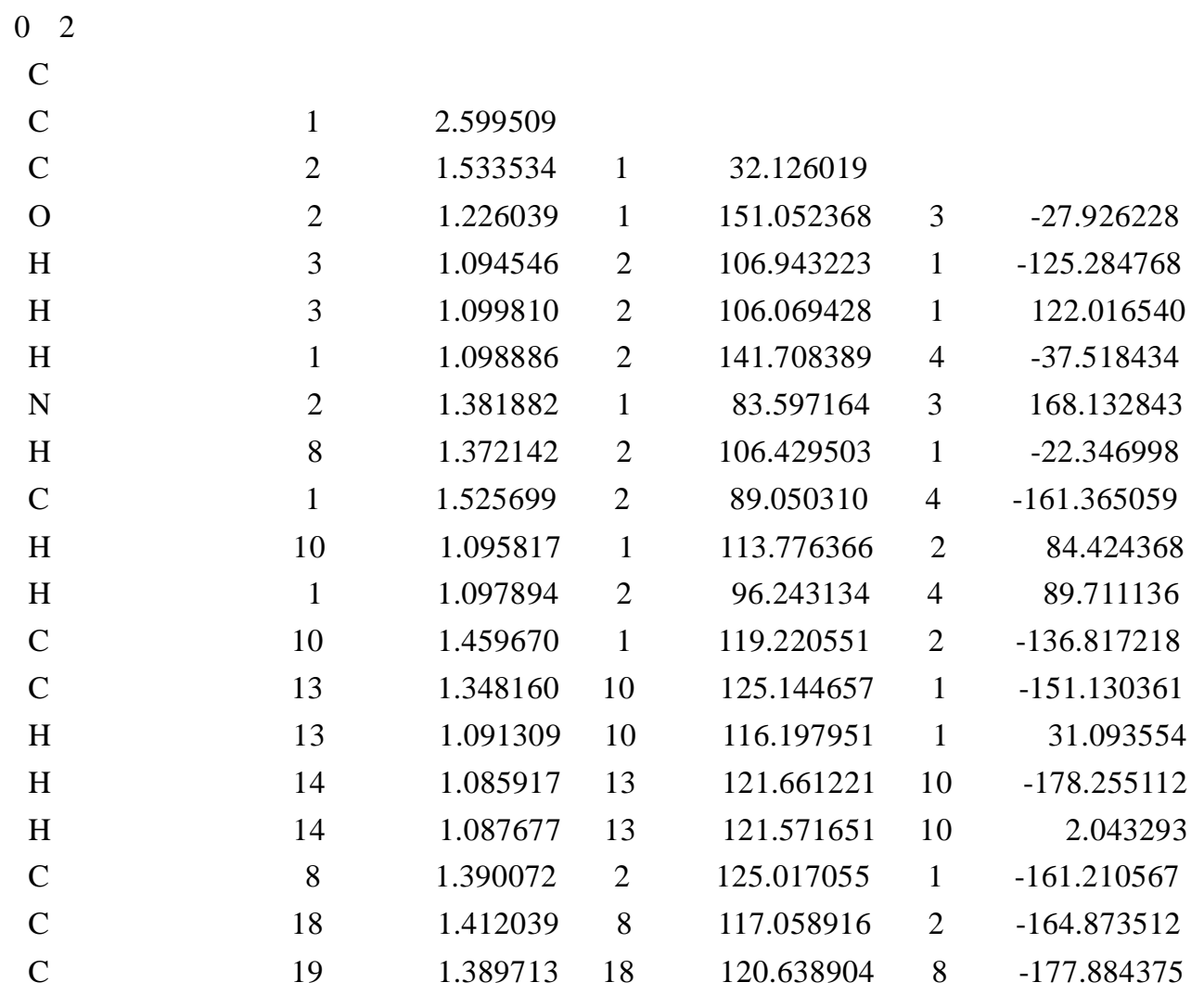




$\begin{array}{lrrrrrr}\mathrm{C} & 20 & 1.398661 & 19 & 120.105087 & 18 & -0.652379 \\ \mathrm{C} & 21 & 1.399369 & 20 & 119.683886 & 19 & 0.348451 \\ \mathrm{C} & 22 & 1.390256 & 21 & 120.804050 & 20 & 0.550280 \\ \mathrm{H} & 19 & 1.086105 & 18 & 118.452616 & 8 & 2.083788 \\ \mathrm{H} & 20 & 1.086663 & 19 & 119.764838 & 18 & 179.802792 \\ \mathrm{H} & 21 & 1.086488 & 20 & 120.176232 & 19 & -179.446795 \\ \mathrm{H} & 22 & 1.086850 & 21 & 119.849113 & 20 & -179.647345 \\ \mathrm{H} & 23 & 1.082551 & 22 & 120.783167 & 21 & -178.781890\end{array}$

$\%$ chk=M-1 $(\mathrm{R}=\mathrm{OMe})$

\# ub3lyp/6-31g* opt=(ts,noeigen,readfc,nofreeze $)$ freq=noraman

calculate for M-1 $\quad \mathrm{HF}=-479.018900$

$\begin{array}{lrrrrrr}\mathrm{Cr}^{2} & & & & & \\ \mathrm{C} & 1 & 2.517681 & & & & \\ \mathrm{C} & 2 & 1.526187 & 1 & 35.300654 & & \\ \mathrm{C} & 2 & 1.217638 & 1 & 134.217830 & 3 & -90.040189 \\ \mathrm{O} & 3 & 1.092211 & 2 & 107.717265 & 1 & -121.732138 \\ \mathrm{H} & 3 & 1.097856 & 2 & 109.677632 & 1 & 120.670288 \\ \mathrm{H} & 1 & 1.098230 & 2 & 144.546033 & 4 & -88.531373 \\ \mathrm{H} & 2 & 1.406902 & 1 & 91.635122 & 3 & 124.881683 \\ \mathrm{~N} & 8 & 1.316694 & 2 & 103.176984 & 1 & 5.952604 \\ \mathrm{H} & 1 & 1.525958 & 2 & 90.974152 & 4 & 142.285127 \\ \mathrm{C} & 10 & 1.096007 & 1 & 113.775396 & 2 & 101.365324 \\ \mathrm{H} & 1 & 1.097865 & 2 & 91.102636 & 4 & 32.748356 \\ \mathrm{H} & 10 & 1.454559 & 1 & 119.592266 & 2 & -118.880295 \\ \mathrm{C} & 13 & 1.350119 & 10 & 125.184469 & 1 & -152.208870 \\ \mathrm{C} & 13 & 1.091128 & 10 & 116.264731 & 1 & 30.056149 \\ \mathrm{H} & 14 & 1.085753 & 13 & 121.612193 & 10 & -177.983093 \\ \mathrm{H} & 14 & 1.087793 & 13 & 121.611039 & 10 & 2.372285 \\ \mathrm{H} & 8 & 1.383195 & 2 & 111.430269 & 1 & 125.373004 \\ \mathrm{O} & 18 & 1.426990 & 8 & 109.631700 & 2 & 154.766406 \\ \mathrm{C} & 19 & 1.092279 & 18 & 105.271094 & 8 & -178.158775 \\ \mathrm{H} & 19 & 1.095154 & 18 & 110.742963 & 8 & -58.868528 \\ \mathrm{H} & 19 & 1.097252 & 18 & 111.048517 & 8 & 63.141827 \\ \mathrm{H} & & & & & & \end{array}$

Table S4. Computational (UB3LYP/6-31G*) Results on Radicals 2 and 3. 


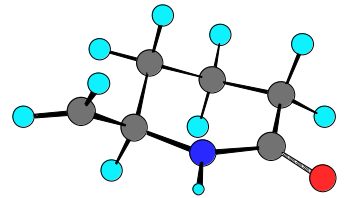

$2(\mathrm{R}=\mathrm{H})$

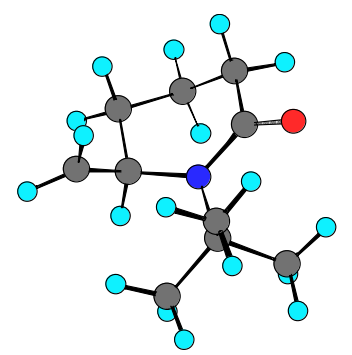

$2(\mathrm{R}=t-\mathrm{Bu})$
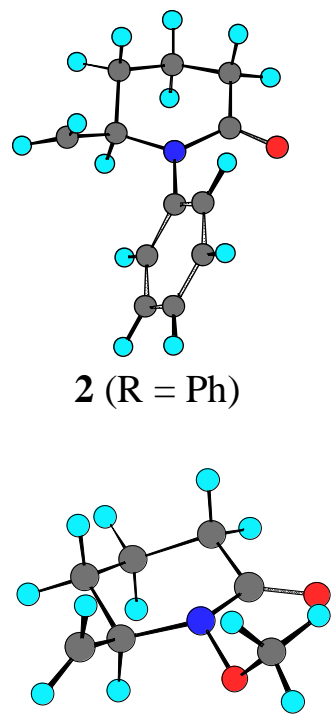

$2(\mathrm{R}=\mathrm{OMe})$

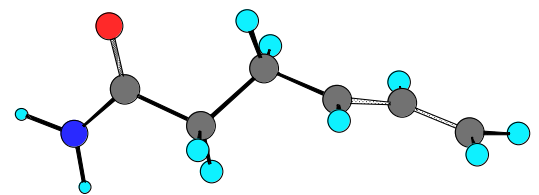

$3(\mathrm{R}=\mathrm{H})$

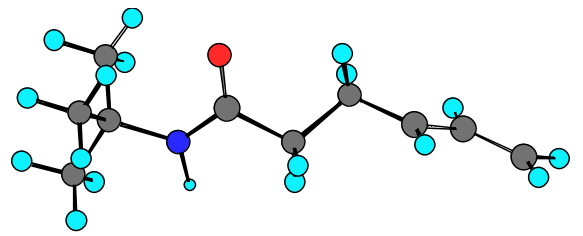

$3(\mathrm{R}=t-\mathrm{Bu})$

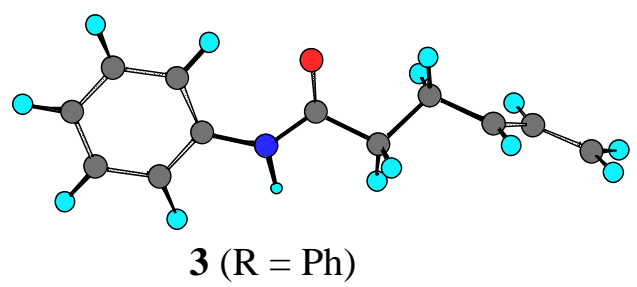

$\%$ chk $=2(\mathrm{R}=\mathrm{H})$

\# ub3lyp/6-31g* opt freq=noraman

calculate for $2 \quad \mathrm{HF}=-364.590285$

$0 \quad 2$

$\mathrm{C}$

C

C

$\mathrm{N}$

C

C

C

$\begin{array}{rrrrr}1.531974 & & & & \\ 1.545876 & 1 & 110.344493 & & \\ 1.468625 & 2 & 109.353821 & 1 & 49.457022 \\ 1.373800 & 3 & 128.316752 & 2 & -26.658058 \\ 1.525943 & 4 & 116.632930 & 3 & 12.834794 \\ 1.492285 & 2 & 112.690786 & 1 & 172.129800\end{array}$




$\begin{array}{lrrrrrr}\mathrm{O} & 5 & 1.224181 & 4 & 121.294084 & 3 & -170.130751 \\ \mathrm{H} & 2 & 1.097843 & 1 & 110.071577 & 6 & 58.721171 \\ \mathrm{H} & 2 & 1.095480 & 1 & 111.416696 & 6 & 177.719657 \\ \mathrm{H} & 1 & 1.098942 & 2 & 109.335753 & 3 & 59.222676 \\ \mathrm{H} & 1 & 1.096391 & 2 & 110.555675 & 3 & 176.286294 \\ \mathrm{H} & 6 & 1.099121 & 5 & 106.460522 & 4 & 99.488531 \\ \mathrm{H} & 6 & 1.093971 & 5 & 106.975623 & 4 & -147.146202 \\ \mathrm{H} & 3 & 1.104208 & 2 & 106.881101 & 1 & -68.777212 \\ \mathrm{H} & 4 & 1.013981 & 3 & 116.823146 & 2 & 168.884429 \\ \mathrm{H} & 7 & 1.084852 & 3 & 120.414574 & 2 & 86.584341 \\ \mathrm{H} & 7 & 1.085819 & 3 & 119.826775 & 2 & -81.696829\end{array}$

\%chk=3 $(\mathrm{R}=\mathrm{H})$

\# ub3lyp/6-31g* opt freq=noraman

calculate for $3 \quad \mathrm{HF}=-364.590879$

$\begin{array}{lrrrrrr}0 \quad 2 & & & & & \\ \mathrm{C} & & & & & & \\ \mathrm{O} & 1 & 1.222638 & & & & \\ \mathrm{~N} & 1 & 1.368522 & 2 & 122.132507 & & \\ \mathrm{C} & 1 & 1.529203 & 2 & 122.707904 & 3 & -179.734994 \\ \mathrm{C} & 4 & 1.541612 & 1 & 111.758740 & 2 & -33.514234 \\ \mathrm{C} & 5 & 1.496457 & 4 & 112.834706 & 1 & 171.144278 \\ \mathrm{C} & 6 & 1.388909 & 5 & 124.817886 & 4 & 120.097850 \\ \mathrm{C} & 7 & 1.385387 & 6 & 125.394999 & 5 & 179.794697 \\ \mathrm{H} & 3 & 1.010644 & 1 & 117.970748 & 2 & -6.700714 \\ \mathrm{H} & 3 & 1.008506 & 1 & 122.311416 & 2 & -173.403531 \\ \mathrm{H} & 4 & 1.096918 & 1 & 111.112345 & 2 & -157.603851 \\ \mathrm{H} & 4 & 1.099559 & 1 & 107.209756 & 2 & 86.340919 \\ \mathrm{H} & 5 & 1.097179 & 4 & 109.394225 & 1 & -65.773139 \\ \mathrm{H} & 5 & 1.098847 & 4 & 106.837764 & 1 & 48.922430 \\ \mathrm{H} & 8 & 1.084788 & 7 & 121.545869 & 6 & -179.852046 \\ \mathrm{H} & 8 & 1.086871 & 7 & 121.260897 & 6 & -0.038523 \\ \mathrm{H} & 7 & 1.091697 & 6 & 117.054075 & 5 & -0.018516 \\ \mathrm{H} & 6 & 1.091334 & 5 & 116.932783 & 4 & -59.781586\end{array}$

$\% \operatorname{chk}=2(\mathrm{R}=t-\mathrm{Bu})$

\# ub3lyp/6-31g* opt freq=noraman

calculate for $2 \quad H F=-521.829150$ 


\begin{tabular}{|c|c|c|c|c|c|c|}
\hline $\mathrm{C}$ & 1 & 1.523317 & & & & \\
\hline $\mathrm{C}$ & 2 & 1.554376 & 1 & 111.900185 & & \\
\hline $\mathrm{N}$ & 3 & 1.490409 & 2 & 113.296374 & 1 & 31.106637 \\
\hline $\mathrm{C}$ & 4 & 1.381709 & 3 & 122.554079 & 2 & 13.577440 \\
\hline $\mathrm{C}$ & 1 & 1.523772 & 2 & 106.536629 & 3 & -62.947732 \\
\hline $\mathrm{C}$ & 3 & 1.499507 & 2 & 108.768832 & 1 & 156.710690 \\
\hline $\mathrm{O}$ & 5 & 1.229423 & 4 & 123.317320 & 3 & 160.456601 \\
\hline $\mathrm{C}$ & 4 & 2.519965 & 3 & 85.430852 & 2 & -163.384286 \\
\hline $\mathrm{C}$ & 4 & 1.516930 & 3 & 118.668463 & 2 & -174.255964 \\
\hline $\mathrm{C}$ & 10 & 1.545534 & 4 & 109.487038 & 3 & 136.347626 \\
\hline $\mathrm{C}$ & 10 & 1.541465 & 4 & 110.385125 & 3 & -101.815614 \\
\hline $\mathrm{H}$ & 2 & 1.097871 & 1 & 108.538431 & 6 & 57.982412 \\
\hline $\mathrm{H}$ & 2 & 1.095545 & 1 & 112.681878 & 6 & 175.869707 \\
\hline $\mathrm{H}$ & 1 & 1.098070 & 2 & 110.610822 & 3 & 57.215496 \\
\hline $\mathrm{H}$ & 1 & 1.096482 & 2 & 110.916649 & 3 & 175.993767 \\
\hline $\mathrm{H}$ & 6 & 1.098577 & 1 & 110.243477 & 2 & -68.038597 \\
\hline $\mathrm{H}$ & 6 & 1.094758 & 1 & 112.938114 & 2 & 173.431860 \\
\hline $\mathrm{H}$ & 3 & 1.095632 & 2 & 106.395652 & 1 & -86.221931 \\
\hline $\mathrm{H}$ & 7 & 1.085949 & 3 & 120.270151 & 2 & 86.330147 \\
\hline $\mathrm{H}$ & 7 & 1.085798 & 3 & 119.645720 & 2 & -78.278039 \\
\hline $\mathrm{H}$ & 9 & 1.093594 & 4 & 94.894740 & 3 & -38.574859 \\
\hline $\mathrm{H}$ & 9 & 1.095895 & 4 & 93.200343 & 3 & 70.755809 \\
\hline $\mathrm{H}$ & 9 & 1.094690 & 4 & 142.057938 & 3 & -165.408318 \\
\hline $\mathrm{H}$ & 11 & 1.088538 & 10 & 111.890125 & 4 & 64.874667 \\
\hline $\mathrm{H}$ & 11 & 1.095878 & 10 & 109.060469 & 4 & -174.797074 \\
\hline $\mathrm{H}$ & 11 & 1.096482 & 10 & 110.330664 & 4 & -56.474272 \\
\hline $\mathrm{H}$ & 12 & 1.096815 & 10 & 109.917611 & 4 & -177.972360 \\
\hline $\mathrm{H}$ & 12 & 1.090895 & 10 & 110.305572 & 4 & -58.071858 \\
\hline $\mathrm{H}$ & 12 & 1.095229 & 10 & 110.769652 & 4 & 62.910834 \\
\hline
\end{tabular}

$\%$ chk=3 $(\mathrm{R}=t-\mathrm{Bu})$

\# ub3lyp/6-31g* opt freq=noraman

calculate for $3 \quad \mathrm{HF}=-521.848072$

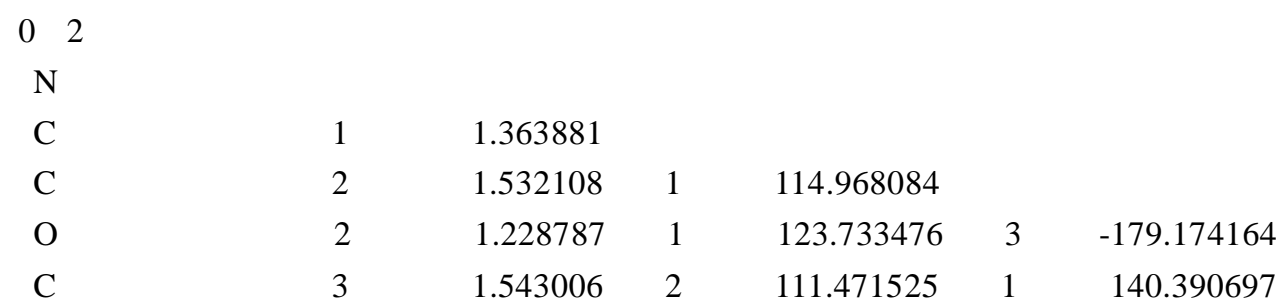




$\begin{array}{lrrrrrr}\mathrm{C} & 5 & 1.496180 & 3 & 112.862847 & 2 & 171.657277 \\ \mathrm{C} & 6 & 1.389005 & 5 & 124.838576 & 3 & 120.111335 \\ \mathrm{C} & 7 & 1.385392 & 6 & 125.378549 & 5 & 179.778330 \\ \mathrm{C} & 1 & 2.474349 & 2 & 104.673429 & 4 & 30.424143 \\ \mathrm{C} & 1 & 1.482330 & 2 & 126.024233 & 4 & -1.816137 \\ \mathrm{C} & 10 & 1.536523 & 1 & 106.391637 & 2 & -179.203312 \\ \mathrm{C} & 10 & 1.540129 & 1 & 110.018068 & 2 & -60.132628 \\ \mathrm{H} & 3 & 1.097187 & 2 & 111.710475 & 1 & 16.350848 \\ \mathrm{H} & 3 & 1.098959 & 2 & 107.201238 & 1 & -100.188418 \\ \mathrm{H} & 5 & 1.098861 & 3 & 106.704418 & 2 & 49.516827 \\ \mathrm{H} & 5 & 1.097568 & 3 & 109.444375 & 2 & -65.279809 \\ \mathrm{H} & 6 & 1.091278 & 5 & 116.911170 & 3 & -59.723559 \\ \mathrm{H} & 8 & 1.084800 & 7 & 121.560412 & 6 & -179.830706 \\ \mathrm{H} & 8 & 1.086860 & 7 & 121.241030 & 6 & -0.035282 \\ \mathrm{H} & 7 & 1.091775 & 6 & 117.061636 & 5 & 0.000184 \\ \mathrm{H} & 9 & 1.091989 & 1 & 91.766655 & 2 & -7.456834 \\ \mathrm{H} & 9 & 1.096049 & 1 & 91.278128 & 2 & 101.240688 \\ \mathrm{H} & 9 & 1.096165 & 1 & 144.407807 & 2 & -134.062680 \\ \mathrm{H} & 11 & 1.097239 & 10 & 111.221342 & 1 & 60.718405 \\ \mathrm{H} & 11 & 1.094414 & 10 & 110.131129 & 1 & -179.837026 \\ \mathrm{H} & 11 & 1.097155 & 10 & 111.218803 & 1 & -60.396141 \\ \mathrm{H} & 12 & 1.096158 & 10 & 110.159927 & 1 & -179.042406 \\ \mathrm{H} & 12 & 1.096100 & 10 & 110.602174 & 1 & -59.450387 \\ \mathrm{H} & 12 & 1.091991 & 10 & 110.401055 & 1 & 60.830577 \\ \mathrm{H} & 1.010292 & 2 & 117.205261 & 4 & -179.855581 \\ & & & & & & \end{array}$

\%chk=2 $(\mathrm{R}=\mathrm{Ph})$

\# ub3lyp/6-31g* opt freq=noraman

calculate for $2 \quad \mathrm{HF}=-595.631509$

$\begin{array}{lrrrrrr}\begin{array}{l}\mathrm{C}^{2} \\ \mathrm{C}\end{array} & & & & & \\ \mathrm{C} & 1 & 1.526555 & & & & \\ \mathrm{C} & 2 & 1.543438 & 1 & 110.805844 & & \\ \mathrm{~N} & 3 & 1.488558 & 2 & 111.319770 & 1 & 49.730588 \\ \mathrm{C} & 4 & 1.391171 & 3 & 124.649840 & 2 & -22.737625 \\ \mathrm{C} & 5 & 1.527128 & 4 & 118.223697 & 3 & 8.254812 \\ \mathrm{C} & 3 & 1.493972 & 2 & 110.966830 & 1 & 173.548266 \\ \mathrm{O} & 5 & 1.223226 & 4 & 122.028870 & 3 & -173.953271 \\ \mathrm{C} & 4 & 1.439319 & 3 & 116.636237 & 2 & 168.444591 \\ \mathrm{C} & 9 & 1.399663 & 4 & 120.309176 & 3 & -131.538492 \\ \mathrm{C} & 10 & 1.393364 & 9 & 120.080605 & 4 & 178.985739\end{array}$




$\begin{array}{lrrrrrr}\mathrm{C} & 11 & 1.396785 & 10 & 120.499933 & 9 & -0.463248 \\ \mathrm{C} & 12 & 1.394595 & 11 & 119.473497 & 10 & 0.380986 \\ \mathrm{C} & 13 & 1.395603 & 12 & 120.261137 & 11 & 0.145697 \\ \mathrm{H} & 2 & 1.098001 & 1 & 109.989408 & 6 & 58.339103 \\ \mathrm{H} & 2 & 1.095769 & 1 & 111.744925 & 6 & 177.225883 \\ \mathrm{H} & 1 & 1.098777 & 2 & 109.698865 & 3 & 58.098198 \\ \mathrm{H} & 1 & 1.096393 & 2 & 110.990313 & 3 & 175.840911 \\ \mathrm{H} & 6 & 1.099332 & 5 & 106.320940 & 4 & 101.120453 \\ \mathrm{H} & 6 & 1.093873 & 5 & 106.004309 & 4 & -146.196910 \\ \mathrm{H} & 3 & 1.102818 & 2 & 106.411624 & 1 & -68.362782 \\ \mathrm{H} & 7 & 1.084565 & 3 & 119.979084 & 2 & 83.606926 \\ \mathrm{H} & 7 & 1.084436 & 3 & 120.344902 & 2 & -85.511816 \\ \mathrm{H} & 10 & 1.083609 & 9 & 119.419696 & 4 & -1.752498 \\ \mathrm{H} & 11 & 1.086751 & 10 & 119.406624 & 9 & 179.761509 \\ \mathrm{H} & 12 & 1.086548 & 11 & 120.266083 & 10 & -179.718363 \\ \mathrm{H} & 13 & 1.086798 & 12 & 120.239756 & 11 & 179.703702 \\ \mathrm{H} & 14 & 1.085714 & 13 & 119.717891 & 12 & 178.999541\end{array}$

\%chk=3 $(\mathrm{R}=\mathrm{Ph})$

\# ub3lyp/6-31g* opt freq=noraman

calculate for $3 \quad \mathrm{HF}=-595.643792$

$\begin{array}{lrrrrrr}0 \quad 2 & & & & \\ \mathrm{~N} & 1 & 1.377136 & & & & \\ \mathrm{C} & 2 & 1.530020 & 1 & 114.135148 & & \\ \mathrm{C} & 2 & 1.223825 & 1 & 124.106651 & 3 & -179.033240 \\ \mathrm{O} & 3 & 1.543067 & 2 & 111.608699 & 1 & 143.060096 \\ \mathrm{C} & 5 & 1.496526 & 3 & 112.735829 & 2 & 171.974158 \\ \mathrm{C} & 6 & 1.388891 & 5 & 124.742700 & 3 & 119.936277 \\ \mathrm{C} & 7 & 1.385324 & 6 & 125.374530 & 5 & 179.659704 \\ \mathrm{C} & 1 & 1.412743 & 2 & 129.287436 & 4 & -1.910415 \\ \mathrm{C} & 9 & 1.404019 & 1 & 117.170627 & 2 & -178.726437 \\ \mathrm{C} & 10 & 1.391933 & 9 & 120.490899 & 1 & -179.976891 \\ \mathrm{C} & 11 & 1.396053 & 10 & 120.240285 & 9 & -0.019930 \\ \mathrm{C} & 12 & 1.395306 & 11 & 119.147663 & 10 & 0.035289 \\ \mathrm{C} & 13 & 1.395204 & 12 & 121.358483 & 11 & -0.003151 \\ \mathrm{C} & 3 & 1.097207 & 2 & 111.680821 & 1 & 19.051000 \\ \mathrm{H} & 3 & 1.099223 & 2 & 107.222200 & 1 & -97.349743 \\ \mathrm{H} & 5 & 1.098687 & 3 & 106.880451 & 2 & 49.827580 \\ \mathrm{H} & 5 & 1.097277 & 3 & 109.406231 & 2 & -65.030888 \\ \mathrm{H} & 6 & 1.091211 & 5 & 116.951731 & 3 & -60.013724 \\ \mathrm{H} & & & & & & \end{array}$




$\begin{array}{crrrrrr}\mathrm{H} & 8 & 1.084756 & 7 & 121.535088 & 6 & -179.826307 \\ \mathrm{H} & 8 & 1.086843 & 7 & 121.266635 & 6 & -0.083624 \\ \mathrm{H} & 7 & 1.091696 & 6 & 117.074283 & 5 & -0.111046 \\ \mathrm{H} & 10 & 1.088741 & 9 & 119.671837 & 1 & 0.046665 \\ \mathrm{H} & 11 & 1.086711 & 10 & 119.401077 & 9 & -179.992689 \\ \mathrm{H} & 12 & 1.086188 & 11 & 120.374173 & 10 & -179.989590 \\ \mathrm{H} & 13 & 1.086899 & 12 & 119.899750 & 11 & 179.964842 \\ \mathrm{H} & 14 & 1.080665 & 13 & 121.284282 & 12 & 179.932013 \\ \mathrm{H} & 1 & 1.010979 & 2 & 115.938523 & 4 & 179.644522\end{array}$

$\% \operatorname{chk}=2(\mathrm{R}=\mathrm{OMe})$

\# ub3lyp/6-31g* opt freq=noraman

calculate for $2 \quad \mathrm{HF}=-479.054841$

$0 \quad 2$

$\mathrm{C}$

$\begin{array}{lll}\mathrm{C} & 1 & 1.529836\end{array}$

$\begin{array}{lllll}\mathrm{C} & 2 & 1.544388 & 1 & 111.452757\end{array}$

$\begin{array}{lllllll}\mathrm{N} & 3 & 1.480515 & 2 & 109.562918 & 1 & 49.790846\end{array}$

$\begin{array}{lllllll}\mathrm{C} & 4 & 1.393011 & 3 & 124.956580 & 2 & -36.547348\end{array}$

$\begin{array}{lllllll}\mathrm{C} & 5 & 1.526112 & 4 & 115.380284 & 3 & 30.001468\end{array}$

$\begin{array}{lllllll}\mathrm{C} & 3 & 1.493607 & 2 & 111.691610 & 1 & 172.203255\end{array}$

$\begin{array}{lllllll}\mathrm{O} & 5 & 1.219780 & 4 & 122.100219 & 3 & -155.535716\end{array}$

$\begin{array}{lllllll}\mathrm{O} & 4 & 1.411101 & 3 & 109.396699 & 2 & -176.646053\end{array}$

$\begin{array}{lllllll}\mathrm{C} & 9 & 1.428777 & 4 & 110.915630 & 3 & -126.813430\end{array}$

$\begin{array}{lllllll}\mathrm{H} & 2 & 1.098028 & 1 & 109.913953 & 6 & 61.198812\end{array}$

$\begin{array}{lllllll}\mathrm{H} & 2 & 1.095518 & 1 & 111.446670 & 6 & 179.716808\end{array}$

$\begin{array}{lllllll}\mathrm{H} & 1 & 1.098568 & 2 & 109.667474 & 3 & 60.965221\end{array}$

$\begin{array}{lllllll}\mathrm{H} & 1 & 1.096335 & 2 & 110.589816 & 3 & 178.424722\end{array}$

$\begin{array}{llllllr}\mathrm{H} & 6 & 1.099230 & 5 & 107.533394 & 4 & 85.613292\end{array}$

$\begin{array}{lllllll}\mathrm{H} & 6 & 1.093403 & 5 & 106.276039 & 4 & -160.283581\end{array}$

$\begin{array}{lllllll}\mathrm{H} & 3 & 1.101081 & 2 & 108.442696 & 1 & -67.651960\end{array}$

$\begin{array}{lllllll}\mathrm{H} & 7 & 1.083701 & 3 & 121.033115 & 2 & 103.538308\end{array}$

$\begin{array}{lllllll}\mathrm{H} & 7 & 1.085450 & 3 & 119.895205 & 2 & -71.955897\end{array}$

$\begin{array}{lllllll}\mathrm{H} & 10 & 1.096768 & 9 & 111.192922 & 4 & 58.498422\end{array}$

$\begin{array}{lllllll}\mathrm{H} & 10 & 1.093381 & 9 & 104.699582 & 4 & 176.883690\end{array}$

$\begin{array}{lllllll}\mathrm{H} & 10 & 1.093400 & 9 & 110.781556 & 4 & -64.796853\end{array}$

$\% \operatorname{chk}=3(\mathrm{R}=\mathrm{OMe})$

\# ub3lyp/6-31g* opt freq=noraman 
calculate for $3 \quad \mathrm{HF}=-479.052946$

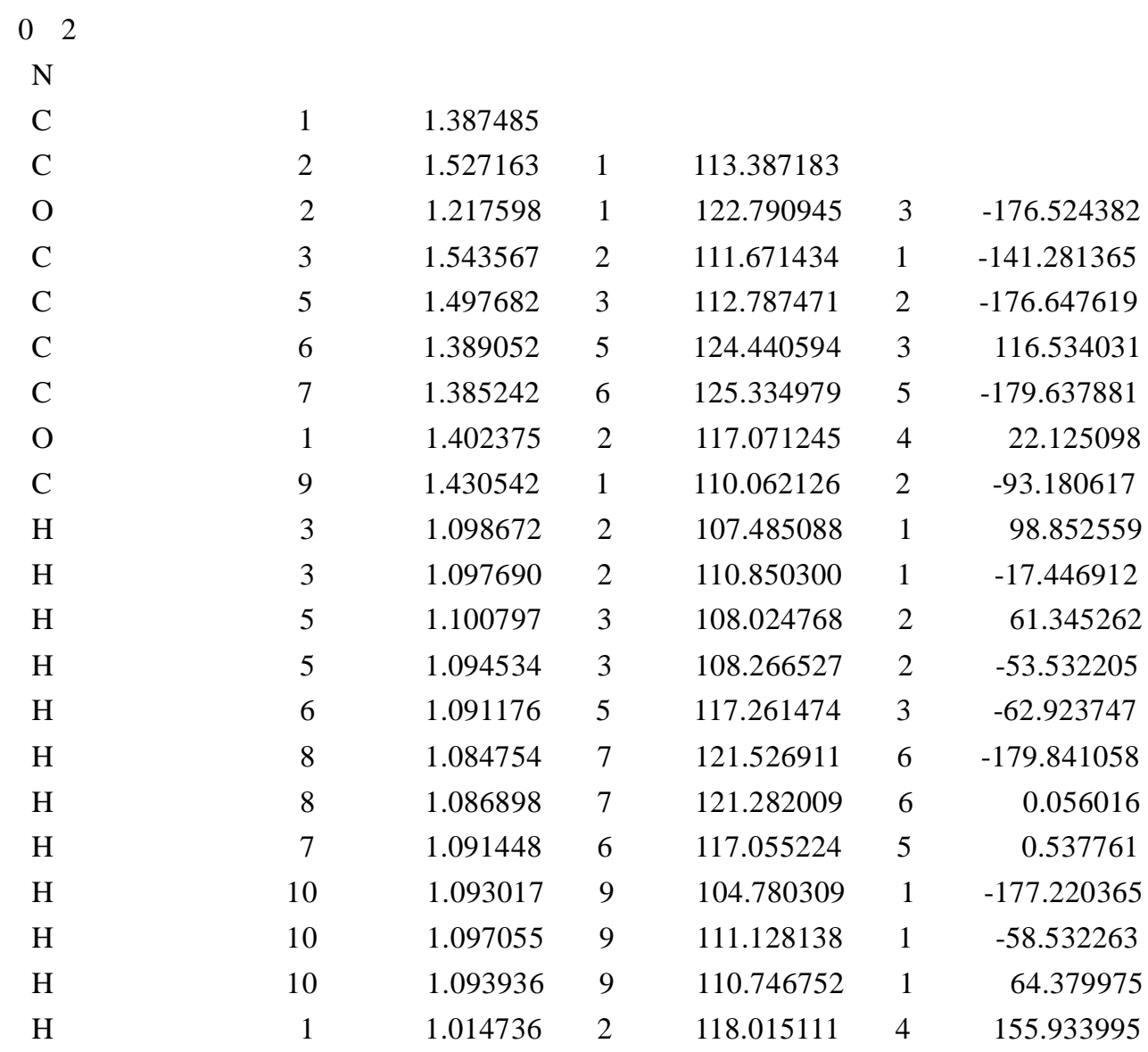

Table S5. Computational (UB3LYP/6-31G*) Results on the Transition State of 7-Endo Cyclization of $\mathbf{1}(\mathrm{R}=\mathrm{H})$.

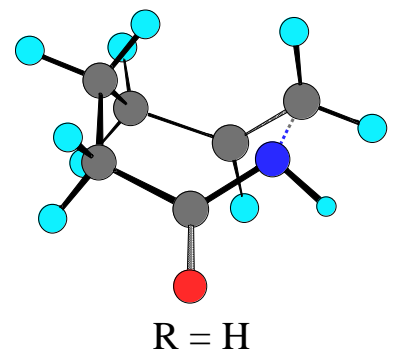

\section{$\%$ chk=7-endo}

\# ub3lyp/6-31g* opt=(ts,noeigen,readfc,nofreeze $)$ freq=noraman

calculate for 7-endo $\quad \mathrm{HF}=-364.544306$

$0 \quad 2$ 
C

C

$\mathrm{N}$

C

C

C

O

C

$\mathrm{H}$

$\mathrm{H}$

$\mathrm{H}$

$\mathrm{H}$

$\mathrm{H}$

$\mathrm{H}$

$\mathrm{H}$

$\mathrm{H}$

$\mathrm{H}$

$\mathrm{H}$

1
1
2
3
5
5
1
2
4
2
4
6
6
3
8
1
8

1.507497

$\begin{array}{lll}2.801775 & 2 & 100.789480\end{array}$

$\begin{array}{lllll}1.543071 & 1 & 113.272930 & 3 & 10.037467\end{array}$

$\begin{array}{lllll}1.377269 & 1 & 87.677103 & 2 & 50.034785\end{array}$

$\begin{array}{lllll}1.528465 & 3 & 118.718857 & 1 & -59.679517\end{array}$

$\begin{array}{lllll}1.233475 & 3 & 120.984819 & 1 & 128.140858\end{array}$

$\begin{array}{lllll}1.360405 & 2 & 122.950139 & 4 & -41.894116\end{array}$

$\begin{array}{lllll}1.097047 & 1 & 109.556126 & 8 & -164.418294\end{array}$

$\begin{array}{lllll}1.095634 & 2 & 110.067414 & 1 & 59.502397\end{array}$

$\begin{array}{lllll}1.101265 & 1 & 109.640210 & 8 & 79.820862\end{array}$

$\begin{array}{lllll}1.097303 & 2 & 108.286586 & 1 & 175.221721\end{array}$

$\begin{array}{lllll}1.099019 & 5 & 105.721290 & 3 & -98.832302\end{array}$

$\begin{array}{lllll}1.096531 & 5 & 106.297635 & 3 & 149.261753\end{array}$

$\begin{array}{lllll}1.025777 & 1 & 107.769354 & 2 & 157.144197\end{array}$

$\begin{array}{lllll}1.086095 & 1 & 120.792569 & 2 & -179.776130\end{array}$

$\begin{array}{lllll}1.088638 & 2 & 117.992502 & 4 & 131.832949\end{array}$

$\begin{array}{lllll}1.083315 & 1 & 121.888684 & 2 & -14.338955\end{array}$

Table S6. Computational (UB3LYP/6-31G*) Results on Radicals 11.
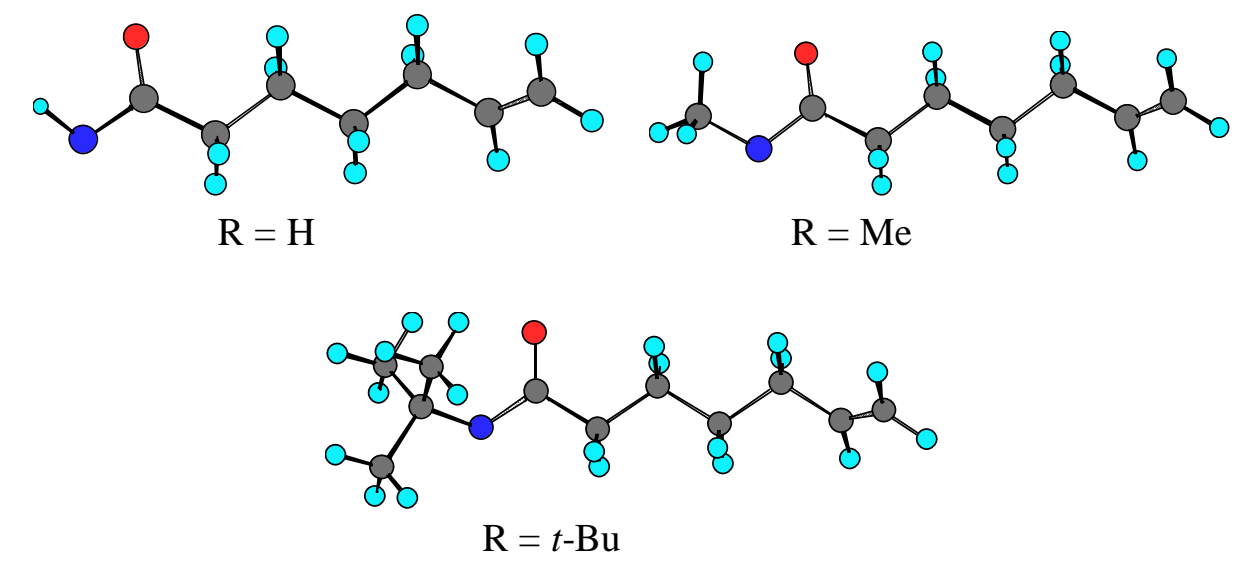

$\%$ chk=11 $(\mathrm{R}=\mathrm{H})$

\# ub3lyp/6-31g* opt freq=noraman

calculate for $11 \quad \mathrm{HF}=-403.864744$

$\begin{array}{ll}0 & 2\end{array}$

$\mathrm{C}$

$\begin{array}{lllll}\mathrm{O} & 1 & 1.237880 & & \\ \mathrm{~N} & 1 & 1.375988 & 2 & 118.449060\end{array}$ 


$\begin{array}{lrrrrrr}\mathrm{C} & 1 & 1.511805 & 2 & 124.027054 & 3 & 172.510135 \\ \mathrm{C} & 4 & 1.530412 & 1 & 113.833822 & 2 & 3.269246 \\ \mathrm{C} & 5 & 1.533336 & 4 & 112.610247 & 1 & 178.872283 \\ \mathrm{C} & 6 & 1.540616 & 5 & 112.838883 & 4 & 179.992008 \\ \mathrm{C} & 7 & 1.504523 & 6 & 112.896956 & 5 & -178.143583 \\ \mathrm{C} & 8 & 1.333415 & 7 & 125.296338 & 6 & -118.991679 \\ \mathrm{H} & 3 & 1.028584 & 1 & 108.359410 & 2 & -30.766572 \\ \mathrm{H} & 4 & 1.098393 & 1 & 107.583466 & 2 & -120.655258 \\ \mathrm{H} & 4 & 1.100399 & 1 & 107.401825 & 2 & 126.647714 \\ \mathrm{H} & 5 & 1.097039 & 4 & 109.059231 & 1 & -58.754742 \\ \mathrm{H} & 5 & 1.097791 & 4 & 109.341042 & 1 & 56.499190 \\ \mathrm{H} & 6 & 1.098884 & 5 & 109.886688 & 4 & -58.182080 \\ \mathrm{H} & 6 & 1.099897 & 5 & 109.530571 & 4 & 58.017500 \\ \mathrm{H} & 7 & 1.101052 & 6 & 108.489308 & 5 & -56.711170 \\ \mathrm{H} & 7 & 1.098047 & 6 & 109.626119 & 5 & 59.229089 \\ \mathrm{H} & 8 & 1.091912 & 7 & 115.720474 & 6 & 60.261428 \\ \mathrm{H} & 9 & 1.086810 & 8 & 121.875343 & 7 & 179.598547 \\ \mathrm{H} & 9 & 1.088482 & 8 & 121.635342 & 7 & -0.692286\end{array}$

\%chk=11 $(\mathrm{R}=\mathrm{Me})$

\# ub3lyp/6-31g* opt freq=noraman

calculate for $11 \quad \mathrm{HF}=-443.183723$

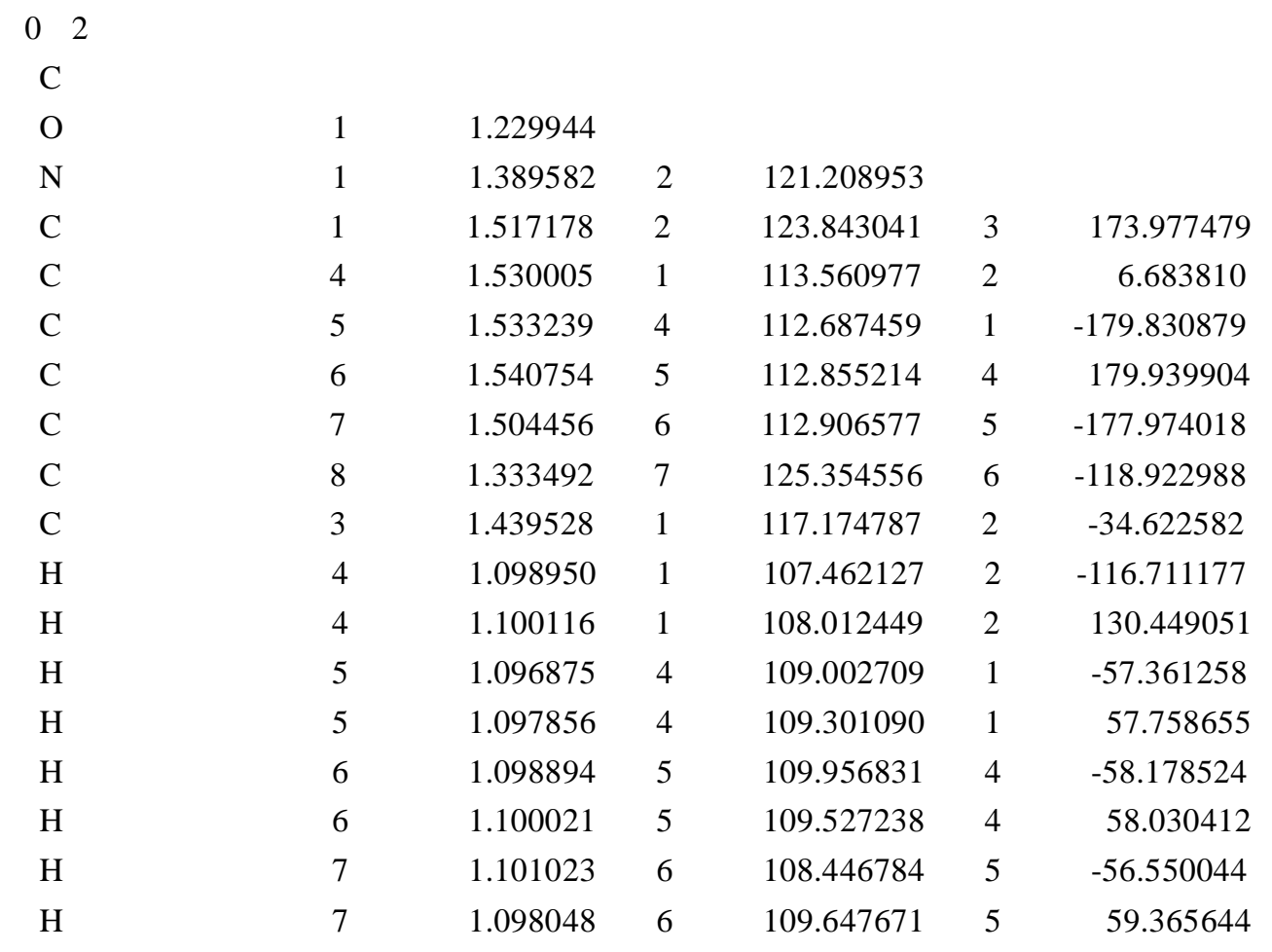




$\begin{array}{crrrrrr}\mathrm{H} & 8 & 1.091980 & 7 & 115.690148 & 6 & 60.310120 \\ \mathrm{H} & 9 & 1.086857 & 8 & 121.874121 & 7 & 179.567472 \\ \mathrm{H} & 9 & 1.088525 & 8 & 121.650493 & 7 & -0.717053 \\ \mathrm{H} & 10 & 1.097661 & 3 & 109.329135 & 1 & 127.172938 \\ \mathrm{H} & 10 & 1.101600 & 3 & 107.631391 & 1 & -118.134939 \\ \mathrm{H} & 10 & 1.095861 & 3 & 113.625415 & 1 & 3.551361\end{array}$

\%chk=11 $(\mathrm{R}=t-\mathrm{Bu})$

\# ub3lyp/6-31g* opt freq=noraman

calculate for $11 \quad \mathrm{HF}=-561.129562$

$0 \quad 2$

\begin{tabular}{|c|c|c|c|c|c|c|}
\hline C & & & & & & \\
\hline $\mathrm{O}$ & 1 & 1.230575 & & & & \\
\hline $\mathrm{N}$ & 1 & 1.381613 & 2 & 121.613899 & & \\
\hline $\mathrm{C}$ & 1 & 1.520643 & 2 & 123.391487 & 3 & 173.439155 \\
\hline $\mathrm{C}$ & 4 & 1.530691 & 1 & 113.399647 & 2 & 3.978408 \\
\hline $\mathrm{C}$ & 5 & 1.533341 & 4 & 112.654390 & 1 & 179.691769 \\
\hline $\mathrm{C}$ & 6 & 1.540809 & 5 & 112.828030 & 4 & 179.882871 \\
\hline $\mathrm{C}$ & 7 & 1.504471 & 6 & 112.926774 & 5 & -178.349556 \\
\hline $\mathrm{C}$ & 8 & 1.333512 & 7 & 125.329169 & 6 & -119.027367 \\
\hline $\mathrm{C}$ & 3 & 2.413209 & 1 & 112.718547 & 2 & -4.982640 \\
\hline $\mathrm{C}$ & 3 & 1.462078 & 1 & 122.433139 & 2 & -46.930114 \\
\hline $\mathrm{C}$ & 11 & 1.541042 & 3 & 106.381464 & 1 & -157.402346 \\
\hline $\mathrm{C}$ & 11 & 1.540723 & 3 & 113.768453 & 1 & -35.485534 \\
\hline $\mathrm{H}$ & 4 & 1.098524 & 1 & 107.669145 & 2 & -119.497359 \\
\hline $\mathrm{H}$ & 4 & 1.100455 & 1 & 107.977122 & 2 & 127.367164 \\
\hline $\mathrm{H}$ & 5 & 1.096835 & 4 & 108.997425 & 1 & -57.859965 \\
\hline $\mathrm{H}$ & 5 & 1.097457 & 4 & 109.298710 & 1 & 57.264492 \\
\hline $\mathrm{H}$ & 6 & 1.098913 & 5 & 109.939364 & 4 & -58.287888 \\
\hline $\mathrm{H}$ & 6 & 1.100001 & 5 & 109.567762 & 4 & 57.940594 \\
\hline $\mathrm{H}$ & 7 & 1.101009 & 6 & 108.464913 & 5 & -56.902242 \\
\hline $\mathrm{H}$ & 7 & 1.098056 & 6 & 109.626975 & 5 & 59.011327 \\
\hline $\mathrm{H}$ & 8 & 1.091982 & 7 & 115.711845 & 6 & 60.226822 \\
\hline $\mathrm{H}$ & 9 & 1.086869 & 8 & 121.887464 & 7 & 179.586211 \\
\hline $\mathrm{H}$ & 9 & 1.088519 & 8 & 121.633743 & 7 & -0.705197 \\
\hline $\mathrm{H}$ & 10 & 1.091657 & 3 & 93.226156 & 1 & 7.167359 \\
\hline $\mathrm{H}$ & 10 & 1.094292 & 3 & 88.462404 & 1 & 115.980173 \\
\hline $\mathrm{H}$ & 10 & 1.096556 & 3 & 144.180754 & 1 & -123.379909 \\
\hline $\mathrm{H}$ & 12 & 1.095516 & 11 & 110.885800 & 3 & 64.834567 \\
\hline $\mathrm{H}$ & 12 & 1.095627 & 11 & 110.119701 & 3 & -175.458330 \\
\hline $\mathrm{H}$ & 12 & 1.094272 & 11 & 110.599000 & 3 & -55.191094 \\
\hline
\end{tabular}




$\begin{array}{lrrrrrr}\mathrm{H} & 13 & 1.095563 & 11 & 110.064610 & 3 & -179.372927 \\ \mathrm{H} & 13 & 1.096613 & 11 & 110.721423 & 3 & -60.404421 \\ \mathrm{H} & 13 & 1.092709 & 11 & 111.156219 & 3 & 60.582247\end{array}$

Table S7. Computational (UB3LYP/6-31G*) Results on the Transition States of Cyclization of $\mathbf{1 1 .}$

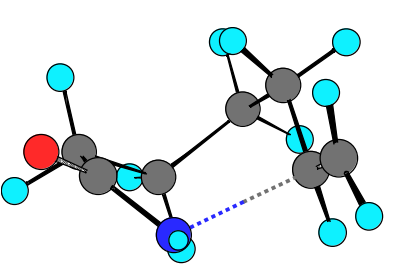

7-exo $(\mathrm{R}=\mathrm{H})$

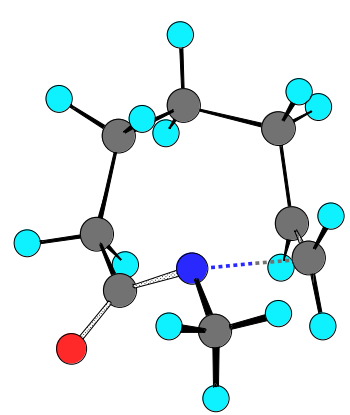

8-endo $(\mathrm{R}=\mathrm{Me})$

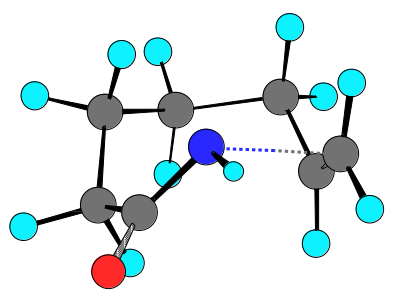

8-endo $(\mathrm{R}=\mathrm{H})$

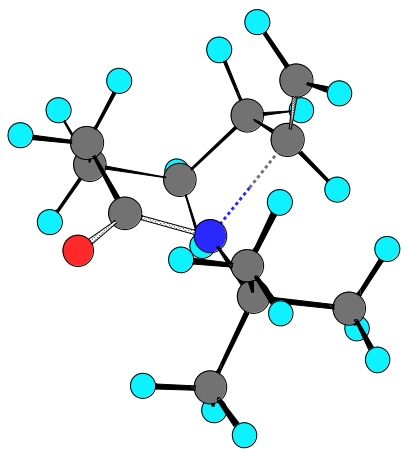

7-exo $(\mathrm{R}=t$-Bu $)$

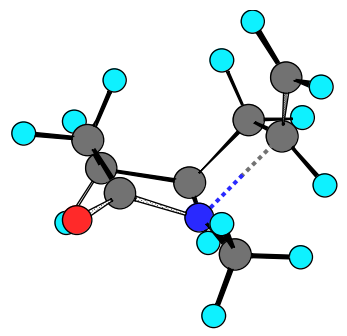

7-exo $(\mathrm{R}=\mathrm{Me})$

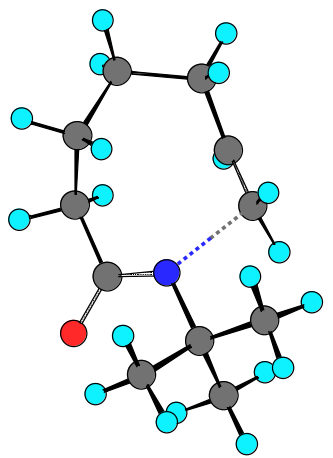

8-endo $(\mathrm{R}=t$ - $\mathrm{Bu})$

\%chk=7-exo $(\mathrm{R}=\mathrm{H})$

\# ub3lyp/6-31g* opt=(ts,noeigen,readfc,nofreeze $)$ freq=noraman

calculate for 7-exo $\quad \mathrm{HF}=-403.855947$

$\begin{array}{ll}0 & 2\end{array}$

$\mathrm{C}$

$\begin{array}{lll}\mathrm{C} & 1 & 1.538526\end{array}$

$\begin{array}{lllll}\mathrm{C} & 2 & 1.537546 & 1 & 116.235128\end{array}$

$\mathrm{N}$

$\mathrm{C}$

$2.843032 \quad 2$

95.836467

$1.376760 \quad 1$

$65.489024 \quad 2$

$-12.247730$

1.526718

$117.007602 \quad 1$

108.104476

$\mathrm{C}$

$1.513356 \quad 2$

$115.869073 \quad 1$

6.920072

O

$1.236422 \quad 4$

55.267134

1.369274

$121.923218 \quad 1$

179.242318

C

7

$121.922828 \quad 2$

$-172.992082$ 


$\begin{array}{llllllr}\mathrm{H} & 2 & 1.098689 & 1 & 108.620738 & 6 & 171.174361 \\ \mathrm{H} & 2 & 1.098451 & 1 & 109.198304 & 6 & -73.714735 \\ \mathrm{H} & 6 & 1.098182 & 5 & 105.539958 & 4 & 108.724164 \\ \mathrm{H} & 6 & 1.097674 & 5 & 107.739319 & 4 & -139.064449 \\ \mathrm{H} & 4 & 1.025294 & 1 & 167.513105 & 2 & 52.724262 \\ \mathrm{H} & 3 & 1.097678 & 2 & 110.654226 & 1 & -69.562163 \\ \mathrm{H} & 3 & 1.099783 & 2 & 107.769245 & 1 & 175.714035 \\ \mathrm{H} & 7 & 1.086692 & 3 & 116.853929 & 2 & 26.866474 \\ \mathrm{H} & 9 & 1.085971 & 7 & 121.455636 & 3 & -170.453063 \\ \mathrm{H} & 9 & 1.087374 & 7 & 121.357904 & 3 & 13.468520 \\ \mathrm{H} & 1 & 1.095014 & 2 & 110.089093 & 3 & -74.798893 \\ \mathrm{H} & 1 & 1.097429 & 2 & 107.907928 & 3 & 169.433982\end{array}$

$\%$ chk=8-endo $(\mathrm{R}=\mathrm{H})$

\# ub3lyp/6-31g* opt=(ts,noeigen,readfc,nofreeze) freq=noraman

calculate for 8-endo $\quad \mathrm{HF}=-403.854736$

$\begin{array}{ll}0 & 2\end{array}$

$\mathrm{C}$

$\begin{array}{lll}\mathrm{C} & 1 & 1.495222\end{array}$

$\begin{array}{lllll}\mathrm{C} & 2 & 1.547669 & 1 & 113.653433\end{array}$

$\begin{array}{lllllll}\mathrm{N} & 1 & 2.897848 & 2 & 104.594047 & 3 & -63.663417\end{array}$

$\begin{array}{lllllll}\mathrm{C} & 3 & 1.540226 & 2 & 115.588521 & 1 & 58.113253\end{array}$

$\begin{array}{lllllll}\mathrm{C} & 4 & 1.373188 & 1 & 93.145125 & 2 & 92.898743\end{array}$

$\begin{array}{lllllll}\mathrm{C} & 6 & 1.526154 & 4 & 119.173208 & 1 & -48.492387\end{array}$

$\begin{array}{lllllll}\mathrm{O} & 6 & 1.236913 & 4 & 120.820314 & 1 & 136.435569\end{array}$

$\begin{array}{lllllll}\mathrm{C} & 1 & 1.364097 & 2 & 123.451055 & 3 & -111.592514\end{array}$

$\begin{array}{llllllr}\mathrm{H} & 2 & 1.097168 & 1 & 109.323157 & 9 & 10.582976\end{array}$

$\begin{array}{lllllll}\mathrm{H} & 3 & 1.099534 & 2 & 109.417890 & 1 & -65.791141\end{array}$

$\begin{array}{lllllll}\mathrm{H} & 5 & 1.095877 & 3 & 109.747048 & 2 & 45.534498\end{array}$

$\begin{array}{lllllll}\mathrm{H} & 2 & 1.100607 & 1 & 109.577677 & 9 & 127.548242\end{array}$

$\begin{array}{lllllll}\mathrm{H} & 3 & 1.097696 & 2 & 107.071740 & 1 & 179.718349\end{array}$

$\begin{array}{lrrrrrr}\mathrm{H} & 5 & 1.096637 & 3 & 107.169406 & 2 & 160.479719\end{array}$

$\begin{array}{lllllll}\mathrm{H} & 7 & 1.094864 & 6 & 104.632491 & 4 & -143.747770\end{array}$

$\begin{array}{lllllll}\mathrm{H} & 7 & 1.100006 & 6 & 106.547905 & 4 & 105.561778\end{array}$

$\begin{array}{lllllll}\mathrm{H} & 4 & 1.026455 & 1 & 118.461174 & 2 & -156.290984\end{array}$

$\begin{array}{llllllr}\mathrm{H} & 1 & 1.090406 & 2 & 117.179861 & 3 & 62.039042\end{array}$

$\begin{array}{lllllll}\mathrm{H} & 9 & 1.086140 & 1 & 120.510078 & 2 & -176.718757\end{array}$

$\begin{array}{lllllll}\mathrm{H} & 9 & 1.084452 & 1 & 120.366160 & 2 & -15.602018\end{array}$

\%chk= 7-exo $(\mathrm{R}=\mathrm{Me})$ 


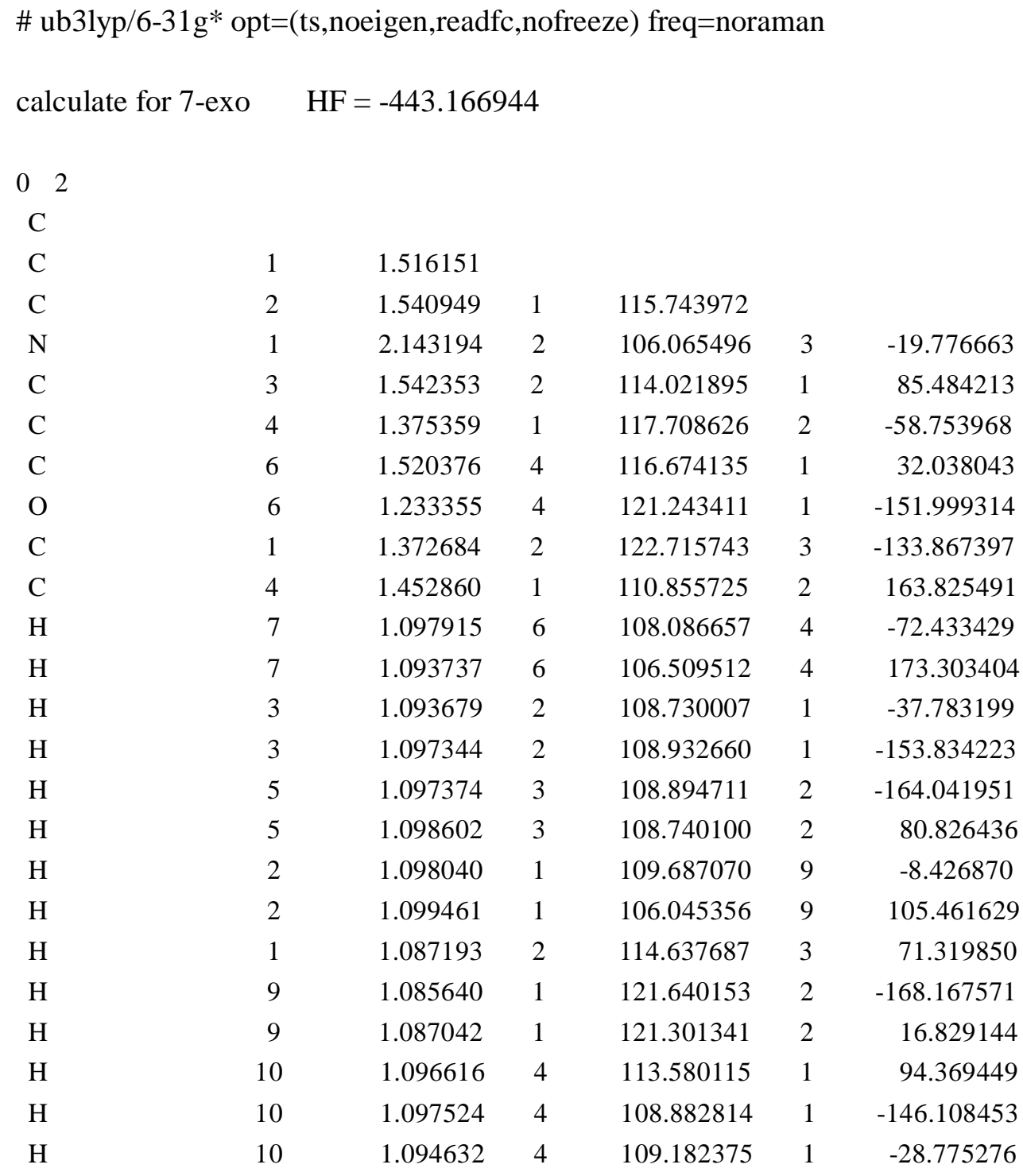

$\%$ chk= 8-endo $(\mathrm{R}=\mathrm{Me})$

\# ub3lyp/6-31g* opt=(ts,noeigen,readfc,nofreeze) freq=noraman

calculate for 8-endo $\quad H F=-443.165732$

$0 \quad 2$

$\begin{array}{lrrrrrr}\mathrm{C} & & & & & & \\ \mathrm{C} & 1 & 1.495749 & & & & \\ \mathrm{C} & 2 & 1.548260 & 1 & 113.316369 & & \\ \mathrm{~N} & 1 & 2.940982 & 2 & 106.603041 & 3 & -63.271425 \\ \mathrm{C} & 3 & 1.538724 & 2 & 114.445836 & 1 & 55.326654 \\ \mathrm{C} & 4 & 1.376211 & 1 & 93.296659 & 2 & 90.612367 \\ \mathrm{C} & 6 & 1.527654 & 4 & 118.793248 & 1 & -39.371282\end{array}$




$\begin{array}{lrrrrrr}\mathrm{O} & 6 & 1.235270 & 4 & 121.187790 & 1 & 145.446214 \\ \mathrm{C} & 1 & 1.369406 & 2 & 123.471716 & 3 & -108.015655 \\ \mathrm{C} & 4 & 1.454064 & 1 & 126.599984 & 2 & -144.267847 \\ \mathrm{H} & 2 & 1.097425 & 1 & 109.252006 & 9 & 13.611955 \\ \mathrm{H} & 3 & 1.099302 & 2 & 109.542186 & 1 & -67.974399 \\ \mathrm{H} & 5 & 1.095759 & 3 & 109.450127 & 2 & 46.555682 \\ \mathrm{H} & 2 & 1.100278 & 1 & 109.807724 & 9 & 130.755187 \\ \mathrm{H} & 3 & 1.097698 & 2 & 107.439849 & 1 & 177.117850 \\ \mathrm{H} & 5 & 1.096425 & 3 & 107.827849 & 2 & 161.800326 \\ \mathrm{H} & 7 & 1.093943 & 6 & 104.523471 & 4 & -155.477099 \\ \mathrm{H} & 7 & 1.098880 & 6 & 107.184534 & 4 & 93.221885 \\ \mathrm{H} & 1 & 1.090134 & 2 & 117.197915 & 3 & 65.223333 \\ \mathrm{H} & 9 & 1.085793 & 1 & 120.020759 & 2 & -175.087315 \\ \mathrm{H} & 9 & 1.084653 & 1 & 119.764017 & 2 & -18.505295 \\ \mathrm{H} & 10 & 1.097295 & 4 & 108.723614 & 1 & 172.593668 \\ \mathrm{H} & 10 & 1.098287 & 4 & 113.830293 & 1 & -68.117394 \\ \mathrm{H} & 10 & 1.094372 & 4 & 109.073226 & 1 & 55.301083\end{array}$

\%chk=7-exo $(\mathrm{R}=t$ - $\mathrm{Bu})$

\# ub3lyp/6-31g* opt=(ts,noeigen,readfc,nofreeze) freq=noraman

calculate for 7-exo $\quad \mathrm{HF}=-561.104504$

$\begin{array}{lrrrrrr}0 \quad 2 & & & & \\ \mathrm{C} & 1 & 1.519825 & & & & \\ \mathrm{C} & 2 & 1.538411 & 1 & 116.170611 & & \\ \mathrm{C} & 1 & 2.108692 & 2 & 108.148205 & 3 & -17.015776 \\ \mathrm{~N} & 3 & 1.539387 & 2 & 112.625546 & 1 & 83.771585 \\ \mathrm{C} & 4 & 1.374799 & 1 & 115.394076 & 2 & -57.486836 \\ \mathrm{C} & 6 & 1.524071 & 4 & 117.567134 & 1 & 25.566480 \\ \mathrm{C} & 6 & 1.236895 & 4 & 122.957501 & 1 & -160.324765 \\ \mathrm{O} & 1 & 1.381931 & 2 & 122.038942 & 3 & -133.644739 \\ \mathrm{C} & 4 & 1.489238 & 1 & 116.671087 & 2 & 153.838912 \\ \mathrm{C} & 10 & 1.540728 & 4 & 110.291319 & 1 & -28.790979 \\ \mathrm{C} & 10 & 1.542510 & 4 & 113.001697 & 1 & 93.329228 \\ \mathrm{C} & 10 & 1.552650 & 4 & 107.402106 & 1 & -144.802439 \\ \mathrm{C} & 7 & 1.095772 & 6 & 109.703510 & 4 & -65.803636 \\ \mathrm{H} & 7 & 1.093254 & 6 & 105.699156 & 4 & 179.350878 \\ \mathrm{H} & 3 & 1.093896 & 2 & 108.977939 & 1 & -39.066520 \\ \mathrm{H} & 3 & 1.097176 & 2 & 109.137994 & 1 & -155.463725 \\ \mathrm{H} & 5 & 1.097250 & 3 & 109.523194 & 2 & -163.397078 \\ \mathrm{H} & 5 & 1.098645 & 3 & 108.927850 & 2 & 80.784943 \\ \mathrm{H} & & & & & & \end{array}$




$\begin{array}{lrrrrrr}\mathrm{H} & 2 & 1.097812 & 1 & 109.817788 & 9 & -8.309840 \\ \mathrm{H} & 2 & 1.099175 & 1 & 105.599101 & 9 & 105.439935 \\ \mathrm{H} & 1 & 1.081697 & 2 & 113.383146 & 3 & 77.360536 \\ \mathrm{H} & 9 & 1.085177 & 1 & 121.515776 & 2 & -164.347019 \\ \mathrm{H} & 9 & 1.086706 & 1 & 121.465497 & 2 & 20.662604 \\ \mathrm{H} & 11 & 1.095232 & 10 & 112.591142 & 4 & 69.587394 \\ \mathrm{H} & 11 & 1.095058 & 10 & 108.854004 & 4 & -171.953470 \\ \mathrm{H} & 11 & 1.095845 & 10 & 111.564444 & 4 & -53.301972 \\ \mathrm{H} & 12 & 1.090583 & 10 & 110.872274 & 4 & 59.491868 \\ \mathrm{H} & 12 & 1.096770 & 10 & 110.058772 & 4 & 179.730593 \\ \mathrm{H} & 12 & 1.096071 & 10 & 110.609692 & 4 & -61.204548 \\ \mathrm{H} & 13 & 1.096723 & 10 & 109.271740 & 4 & 175.136138 \\ \mathrm{H} & 13 & 1.089391 & 10 & 110.752294 & 4 & -64.438244 \\ \mathrm{H} & 13 & 1.095636 & 10 & 110.289362 & 4 & 56.284208\end{array}$

$\%$ chk $=8$-endo $(\mathrm{R}=t$-Bu $)$

\# ub31yp/6-31 $\mathrm{g} * \mathrm{opt}=(\mathrm{ts}$, noeigen,readfc,nofreeze) freq=noraman

calculate for 8-endo $\quad \mathrm{HF}=-561.103606$

$\begin{array}{lrrrrrr}\text { O } 2 & & & & \\ \mathrm{C} & & & & & \\ \mathrm{C} & 1 & 1.496533 & & & & \\ \mathrm{C} & 2 & 1.548919 & 1 & 113.366257 & & \\ \mathrm{~N} & 1 & 2.985032 & 2 & 109.843187 & 3 & -60.413921 \\ \mathrm{C} & 3 & 1.536902 & 2 & 113.262375 & 1 & 51.353103 \\ \mathrm{C} & 4 & 1.373125 & 1 & 90.971587 & 2 & 89.025191 \\ \mathrm{C} & 6 & 1.531843 & 4 & 118.918085 & 1 & -36.872321 \\ \mathrm{O} & 6 & 1.238769 & 4 & 123.028393 & 1 & 148.867440 \\ \mathrm{C} & 1 & 1.375211 & 2 & 123.170099 & 3 & -104.279057 \\ \mathrm{C} & 4 & 1.485659 & 1 & 132.507722 & 2 & -136.953788 \\ \mathrm{C} & 10 & 1.541949 & 4 & 113.960953 & 1 & -66.820145 \\ \mathrm{C} & 10 & 1.542722 & 4 & 108.928555 & 1 & 56.083715 \\ \mathrm{C} & 10 & 1.553303 & 4 & 107.197491 & 1 & 171.146885 \\ \mathrm{H} & 2 & 1.097644 & 1 & 109.021783 & 9 & 16.776680 \\ \mathrm{H} & 3 & 1.098859 & 2 & 109.768577 & 1 & -71.347423 \\ \mathrm{H} & 5 & 1.095805 & 3 & 109.481508 & 2 & 46.419812 \\ \mathrm{H} & 2 & 1.099690 & 1 & 109.896321 & 9 & 133.889136 \\ \mathrm{H} & 3 & 1.097542 & 2 & 107.672974 & 1 & 173.322708 \\ \mathrm{H} & 5 & 1.096325 & 3 & 108.445630 & 2 & 162.113172 \\ \mathrm{H} & 7 & 1.093574 & 6 & 103.954060 & 4 & -158.053722 \\ \mathrm{H} & 7 & 1.097774 & 6 & 107.642107 & 4 & 90.753708 \\ \mathrm{H} & 1 & 1.089772 & 2 & 117.454812 & 3 & 70.178216\end{array}$




$\begin{array}{lrrrrrr}\mathrm{H} & 9 & 1.084249 & 1 & 119.320589 & 2 & -171.026972 \\ \mathrm{H} & 9 & 1.082433 & 1 & 118.476209 & 2 & -18.832481 \\ \mathrm{H} & 11 & 1.096917 & 10 & 111.221837 & 4 & 61.328480 \\ \mathrm{H} & 11 & 1.096651 & 10 & 110.031018 & 4 & -179.882691 \\ \mathrm{H} & 11 & 1.090806 & 10 & 110.671976 & 4 & -59.603869 \\ \mathrm{H} & 12 & 1.094059 & 10 & 112.665116 & 4 & -72.855456 \\ \mathrm{H} & 12 & 1.095560 & 10 & 110.790288 & 4 & 49.093829 \\ \mathrm{H} & 12 & 1.095139 & 10 & 109.362972 & 4 & 168.193492 \\ \mathrm{H} & 13 & 1.096501 & 10 & 109.150608 & 4 & -172.533670 \\ \mathrm{H} & 13 & 1.095427 & 10 & 110.248287 & 4 & -53.750369 \\ \mathrm{H} & 13 & 1.089475 & 10 & 110.866626 & 4 & 67.001081\end{array}$

Table S8. Computational (UB3LYP/6-31G*) Results on the Transition States of 1,5-H Abstraction of $\mathbf{1 1 .}$

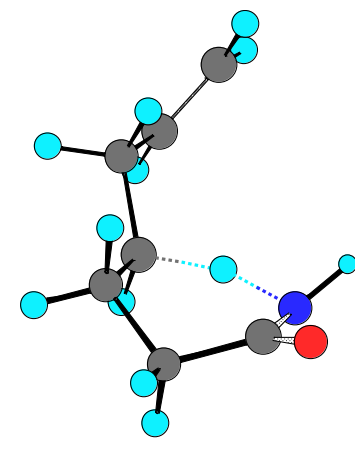

$\mathrm{R}=\mathrm{H}$

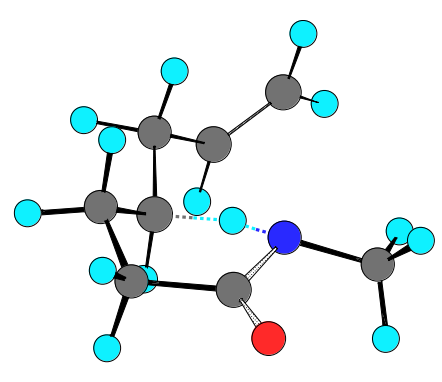

$\mathrm{R}=\mathrm{Me}$

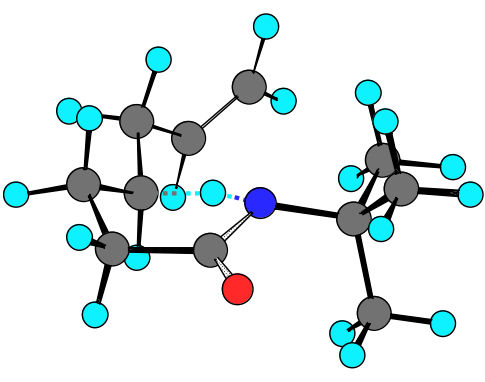

$\mathrm{R}=t-\mathrm{Bu}$

$\%$ chk=M-11 $(\mathrm{R}=\mathrm{H})$

\# ub3lyp/6-31g* opt=(ts,noeigen,readfc,nofreeze) freq=noraman

calculate for $\mathrm{M}-11 \quad \mathrm{HF}=-403.846326$

$0 \quad 2$

$\mathrm{C}$

$\mathrm{C}$

$\mathrm{C}$

$\mathrm{O}$

$\mathrm{H}$

$\mathrm{H}$

$\mathrm{H}$

N

$\mathrm{H}$

C

$\begin{array}{rrrrr}2.515360 & & & & \\ 1.526455 & 1 & 35.285016 & & \\ 1.226325 & 1 & 137.607694 & 3 & -83.473979 \\ 1.092363 & 2 & 108.281311 & 1 & -122.232544 \\ 1.097941 & 2 & 108.925539 & 1 & 120.294231 \\ 1.098925 & 2 & 144.891046 & 4 & -85.723068 \\ 1.382082 & 1 & 91.635407 & 3 & 130.478569 \\ 1.367266 & 2 & 105.036804 & 1 & 2.737423 \\ 1.526047 & 2 & 89.767656 & 4 & 146.935851\end{array}$




$\begin{array}{lrrrrrr}\mathrm{H} & 10 & 1.097561 & 1 & 112.637909 & 2 & 101.668801 \\ \mathrm{H} & 1 & 1.098387 & 2 & 92.121245 & 4 & 37.506799 \\ \mathrm{C} & 10 & 1.521226 & 1 & 117.249601 & 2 & -124.593100 \\ \mathrm{C} & 13 & 1.505705 & 10 & 113.697132 & 1 & 177.953476 \\ \mathrm{H} & 13 & 1.105314 & 10 & 107.649718 & 1 & -60.896986 \\ \mathrm{H} & 14 & 1.091233 & 13 & 115.813880 & 10 & 57.647652 \\ \mathrm{H} & 8 & 1.023618 & 2 & 112.021465 & 1 & 124.047923 \\ \mathrm{H} & 13 & 1.097916 & 10 & 109.838557 & 1 & 54.041362 \\ \mathrm{C} & 14 & 1.332923 & 13 & 124.910814 & 10 & -121.923415 \\ \mathrm{H} & 19 & 1.088399 & 14 & 121.718647 & 13 & -0.407691 \\ \mathrm{H} & 19 & 1.086544 & 14 & 121.785880 & 13 & 179.855234\end{array}$

$\%$ chk=M-11 $(\mathrm{R}=\mathrm{Me})$

\# ub3lyp/6-31g* opt=(ts,noeigen,readfc,nofreeze) freq=noraman

calculate for $\mathrm{M}-11 \quad \mathrm{HF}=-443.160024$

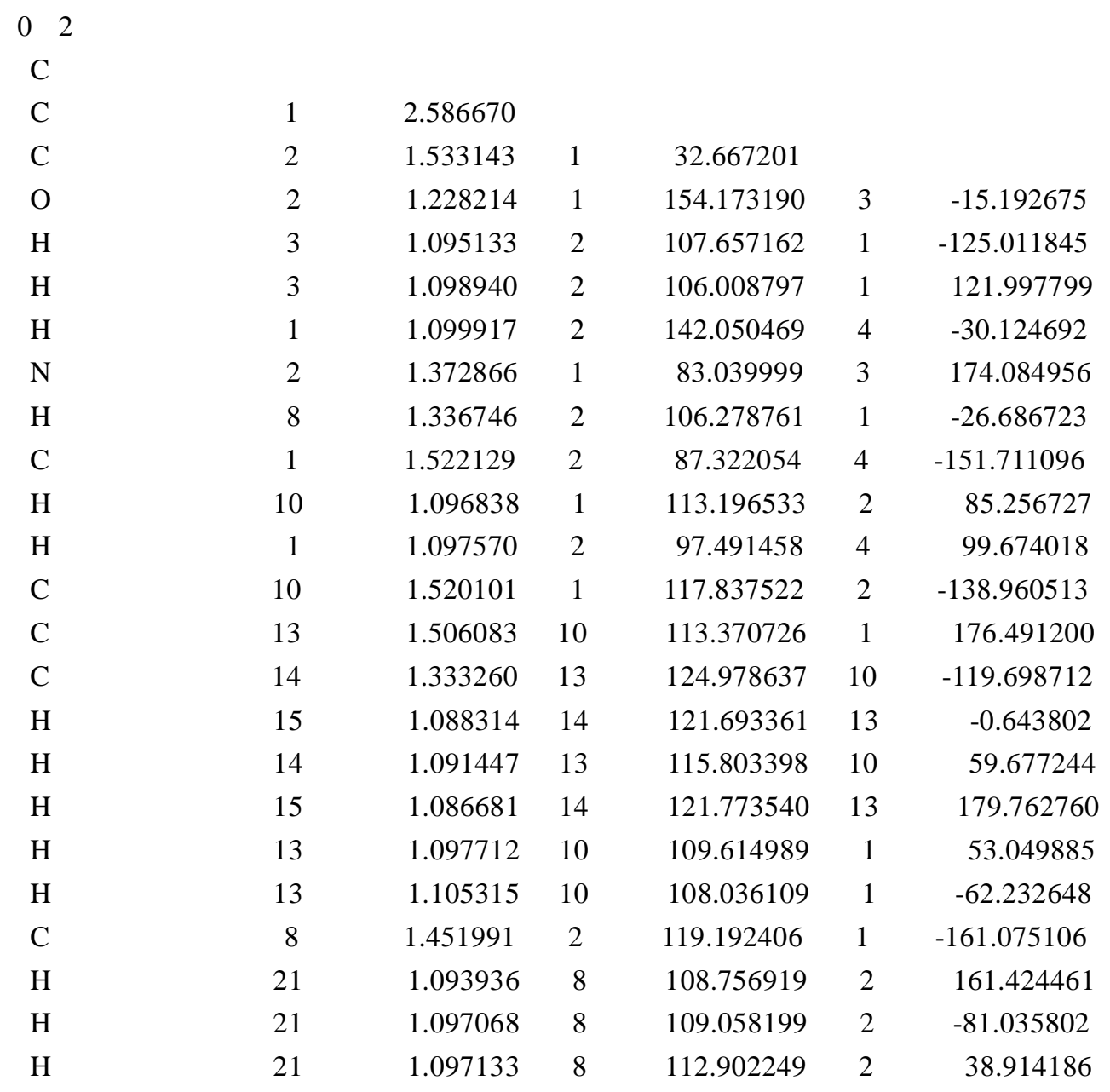




\begin{tabular}{|c|c|c|c|c|c|c|}
\hline$\% \operatorname{chk}=\mathrm{M}-11(\mathrm{R}=t-\mathrm{Bu})$ & \multicolumn{6}{|c|}{ \# ub3lyp/6-31g* opt=(ts,noeigen,readfc,nofreeze) freq=noraman } \\
\hline \multicolumn{2}{|c|}{ calculate for M-11 } & \multicolumn{5}{|c|}{$\mathrm{HF}=-561.106078$} \\
\hline \multicolumn{7}{|c|}{02} \\
\hline \multicolumn{7}{|c|}{$\mathrm{C}$} \\
\hline $\mathrm{C}$ & 1 & 2.589652 & & & & \\
\hline $\mathrm{C}$ & 1 & 1.534083 & 2 & 32.479565 & & \\
\hline $\mathrm{O}$ & 2 & 1.231609 & 1 & 151.780718 & 3 & -20.816367 \\
\hline $\mathrm{H}$ & 3 & 1.094852 & 1 & 111.864227 & 2 & 122.521790 \\
\hline $\mathrm{H}$ & 3 & 1.099274 & 1 & 110.189849 & 2 & -119.897482 \\
\hline $\mathrm{H}$ & 1 & 1.100348 & 2 & 142.295363 & 4 & -33.693336 \\
\hline $\mathrm{N}$ & 2 & 1.369906 & 1 & 83.438575 & 3 & 171.697460 \\
\hline $\mathrm{H}$ & 8 & 1.344318 & 2 & 106.510854 & 1 & -25.287137 \\
\hline $\mathrm{C}$ & 1 & 1.520814 & 2 & 87.761446 & 4 & -155.795211 \\
\hline $\mathrm{H}$ & 10 & 1.096983 & 1 & 112.783484 & 2 & 84.491294 \\
\hline $\mathrm{H}$ & 1 & 1.097852 & 2 & 96.923001 & 4 & 95.357579 \\
\hline $\mathrm{C}$ & 10 & 1.520642 & 1 & 117.203632 & 2 & -141.227587 \\
\hline $\mathrm{C}$ & 13 & 1.505176 & 10 & 114.304506 & 1 & -174.194777 \\
\hline $\mathrm{C}$ & 14 & 1.333500 & 13 & 125.261150 & 10 & -125.999171 \\
\hline $\mathrm{H}$ & 15 & 1.088176 & 14 & 121.711510 & 13 & 0.583314 \\
\hline $\mathrm{H}$ & 14 & 1.091607 & 13 & 115.606947 & 10 & 54.669967 \\
\hline $\mathrm{H}$ & 15 & 1.086663 & 14 & 121.764008 & 13 & -179.112012 \\
\hline $\mathrm{H}$ & 13 & 1.097930 & 10 & 110.027836 & 1 & 60.976597 \\
\hline $\mathrm{H}$ & 13 & 1.106013 & 10 & 107.232658 & 1 & -53.666923 \\
\hline $\mathrm{C}$ & 8 & 2.426350 & 2 & 160.524413 & 1 & -150.567069 \\
\hline $\mathrm{C}$ & 8 & 1.476973 & 2 & 123.944423 & 1 & -163.602131 \\
\hline $\mathrm{H}$ & 21 & 1.095547 & 8 & 93.126203 & 2 & 105.959649 \\
\hline $\mathrm{H}$ & 21 & 1.095548 & 8 & 89.704345 & 2 & -145.915770 \\
\hline $\mathrm{H}$ & 21 & 1.095403 & 8 & 145.451051 & 2 & -22.803948 \\
\hline $\mathrm{C}$ & 22 & 1.547122 & 8 & 107.919816 & 2 & -69.907404 \\
\hline $\mathrm{C}$ & 22 & 1.540152 & 8 & 111.716977 & 2 & 51.834498 \\
\hline $\mathrm{H}$ & 26 & 1.090662 & 22 & 110.554121 & 8 & 64.184647 \\
\hline $\mathrm{H}$ & 26 & 1.096784 & 22 & 109.299822 & 8 & -175.276611 \\
\hline $\mathrm{H}$ & 26 & 1.095103 & 22 & 110.354357 & 8 & -56.151771 \\
\hline $\mathrm{H}$ & 27 & 1.096208 & 22 & 110.387579 & 8 & -179.655505 \\
\hline $\mathrm{H}$ & 27 & 1.092689 & 22 & 110.557523 & 8 & -59.545629 \\
\hline $\mathrm{H}$ & 27 & 1.096703 & 22 & 110.645607 & 8 & 61.050424 \\
\hline
\end{tabular}

Table S9. Computational (UB3LYP/6-31G*) Results on the Transition States of 1,6-H Abstraction of 11 . 


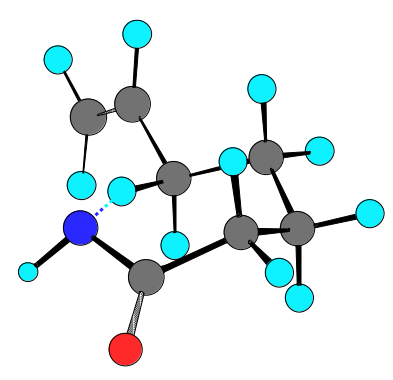

$\mathrm{R}=\mathrm{H}$

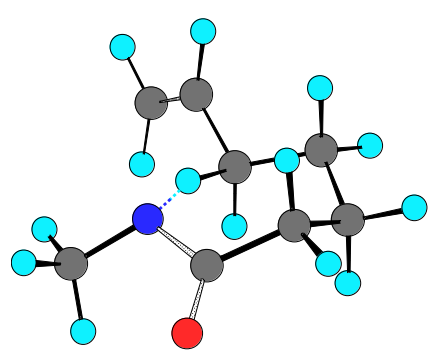

$\mathrm{R}=\mathrm{Me}$

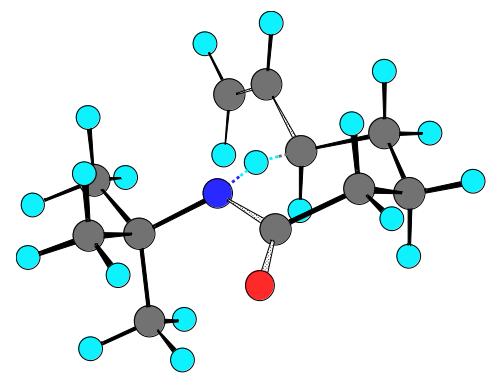

$\mathrm{R}=t-\mathrm{Bu}$

$\%$ chk=1,6-H $(\mathrm{R}=\mathrm{H})$

\# ub3lyp/6-31g* opt=(ts,noeigen,readfc,nofreeze) freq=noraman

calculate for $1,6-\mathrm{H} \quad \mathrm{HF}=-403.855819$

$0 \quad 2$
2

$\mathrm{C}$

C

C

C

$\mathrm{C}$

C

$\mathrm{O}$

$\mathrm{H}$

$\mathrm{H}$

$\mathrm{H}$

$\mathrm{H}$

$\mathrm{H}$

$\mathrm{H}$

$\mathrm{H}$

$\mathrm{H}$

$\mathrm{H}$

C

$\mathrm{H}$

$\mathrm{H}$

$\mathrm{N}$

$\mathrm{H}$
1.537564

$\begin{array}{lll}1.529419 & 1 & 114.634656\end{array}$

$\begin{array}{lllll}2.587852 & 2 & 103.696015 & 3 & -38.190789\end{array}$

$\begin{array}{lllll}1.522038 & 1 & 32.851306 & 2 & -116.855421\end{array}$

$\begin{array}{lllll}1.476657 & 2 & 116.702406 & 1 & 169.760211\end{array}$

$\begin{array}{lllll}1.231449 & 1 & 130.123676 & 2 & 153.849704\end{array}$

$\begin{array}{lllll}1.099377 & 1 & 109.306975 & 5 & 52.964035\end{array}$

$\begin{array}{lllll}1.099907 & 1 & 108.740722 & 5 & 168.397336\end{array}$

$\begin{array}{lllll}1.098931 & 2 & 109.798114 & 3 & 54.474184\end{array}$

$\begin{array}{lllll}1.096817 & 2 & 108.080736 & 3 & 169.662332\end{array}$

$\begin{array}{lllll}1.097835 & 4 & 108.749612 & 1 & 122.787167\end{array}$

$\begin{array}{lllll}1.093654 & 4 & 106.430526 & 1 & -121.137162\end{array}$

$\begin{array}{lllll}1.096947 & 2 & 112.059226 & 1 & -58.952055\end{array}$

$\begin{array}{lllll}1.090996 & 3 & 116.106310 & 2 & -40.764947\end{array}$

$\begin{array}{lllll}1.212513 & 2 & 103.406354 & 1 & 52.584722\end{array}$

$\begin{array}{lllll}1.341871 & 3 & 124.923628 & 2 & 140.733204\end{array}$

$\begin{array}{lllll}1.087976 & 6 & 121.642527 & 3 & -1.236742\end{array}$

$\begin{array}{lllll}1.086248 & 6 & 121.712617 & 3 & 179.042795\end{array}$

$\begin{array}{lllll}1.377825 & 1 & 102.035319 & 2 & 4.043814\end{array}$

$\begin{array}{lllll}1.025577 & 4 & 109.368742 & 1 & 130.181952\end{array}$

\%chk=1,6-H $(\mathrm{R}=\mathrm{Me})$

\# ub3lyp/6-31g* opt=(ts,noeigen,readfc,nofreeze) freq=noraman

calculate for $1,6-\mathrm{H} \quad \mathrm{HF}=-443.169181$ 


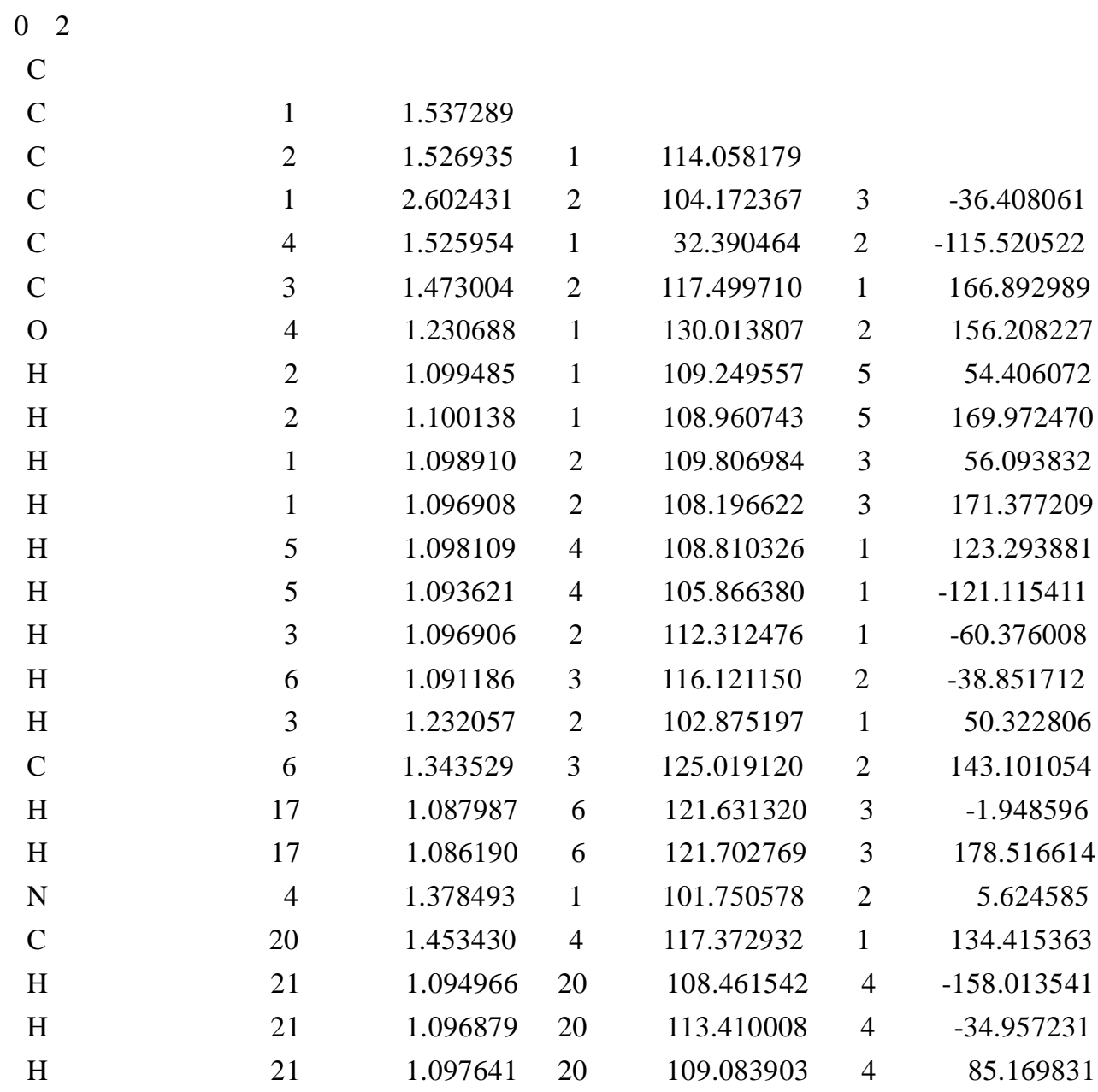

$\%$ chk $=1,6-\mathrm{H}(\mathrm{R}=t$-Bu $)$

\# ub3lyp/6-31g* opt=(ts,noeigen,readfc,nofreeze $)$ freq=noraman

calculate for $1,6-\mathrm{H} \quad \mathrm{HF}=-561.111770$

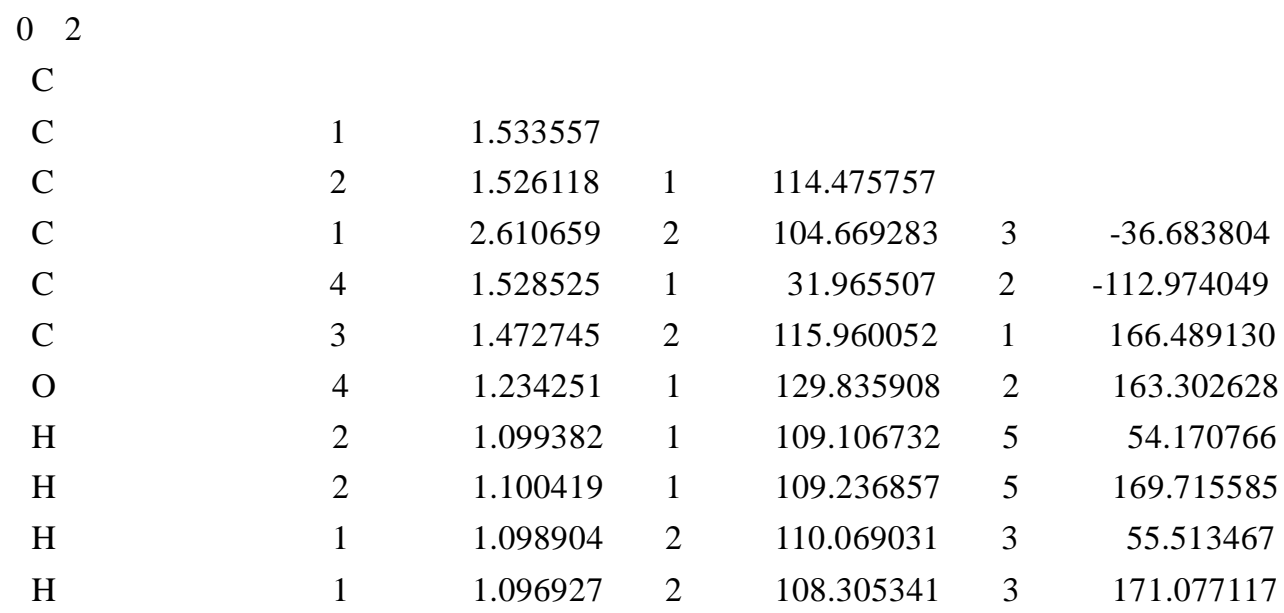




$\begin{array}{lrrrrrr}\mathrm{H} & 5 & 1.098362 & 4 & 108.777306 & 1 & 123.781722 \\ \mathrm{H} & 5 & 1.093544 & 4 & 105.121274 & 1 & -121.236070 \\ \mathrm{H} & 3 & 1.096640 & 2 & 112.160805 & 1 & -62.425800 \\ \mathrm{H} & 6 & 1.091197 & 3 & 116.002345 & 2 & -43.444075 \\ \mathrm{H} & 3 & 1.262311 & 2 & 103.803456 & 1 & 47.745522 \\ \mathrm{C} & 6 & 1.343278 & 3 & 125.223398 & 2 & 136.733252 \\ \mathrm{H} & 17 & 1.088001 & 6 & 121.660084 & 3 & -0.504116 \\ \mathrm{H} & 17 & 1.086292 & 6 & 121.703801 & 3 & 179.997702 \\ \mathrm{~N} & 4 & 1.373856 & 1 & 101.851423 & 2 & 9.516946 \\ \mathrm{C} & 20 & 2.432908 & 4 & 158.988079 & 1 & 132.916668 \\ \mathrm{C} & 20 & 1.480864 & 4 & 122.069356 & 1 & 139.965629 \\ \mathrm{H} & 21 & 1.095362 & 20 & 88.651949 & 4 & 139.844637 \\ \mathrm{H} & 21 & 1.093677 & 20 & 94.720245 & 4 & -112.209646 \\ \mathrm{H} & 21 & 1.095425 & 20 & 144.993830 & 4 & 18.784702 \\ \mathrm{C} & 22 & 1.540596 & 20 & 112.616756 & 4 & -54.406666 \\ \mathrm{C} & 22 & 1.549028 & 20 & 108.020985 & 4 & 67.893635 \\ \mathrm{H} & 26 & 1.092005 & 22 & 110.735112 & 20 & 60.187814 \\ \mathrm{H} & 26 & 1.096324 & 22 & 110.254070 & 20 & -179.622179 \\ \mathrm{H} & 26 & 1.096809 & 22 & 110.715375 & 20 & -60.533123 \\ \mathrm{H} & 27 & 1.096757 & 22 & 109.103225 & 20 & 172.540244 \\ \mathrm{H} & 27 & 1.090173 & 22 & 110.884896 & 20 & -66.910209 \\ \mathrm{H} & 27 & 1.095241 & 22 & 110.304851 & 20 & 53.727397\end{array}$

Table S10. ${ }^{1} \mathrm{H}$ or ${ }^{13} \mathrm{C}$ NMR Spectra of 4,5 and 7. 


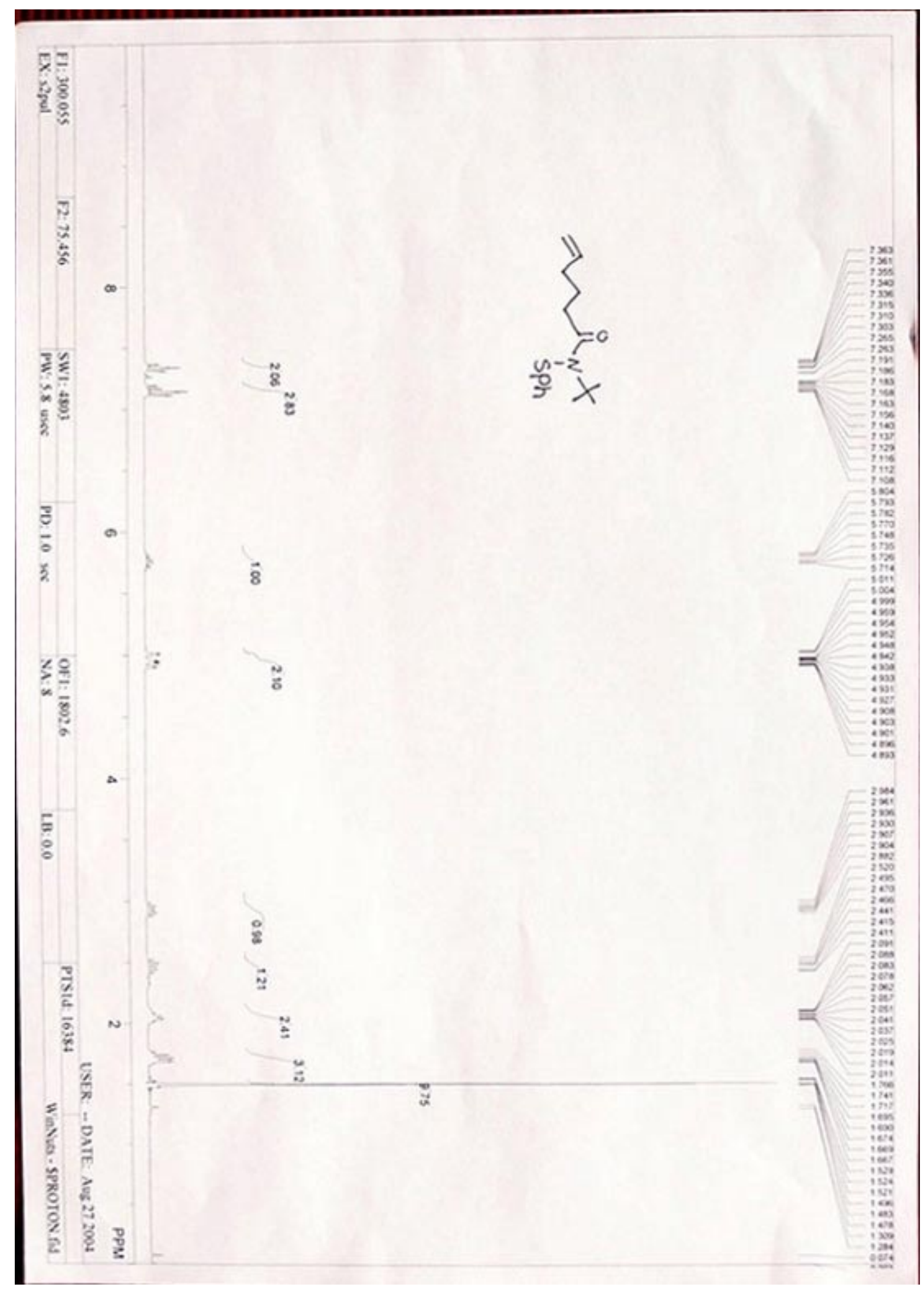




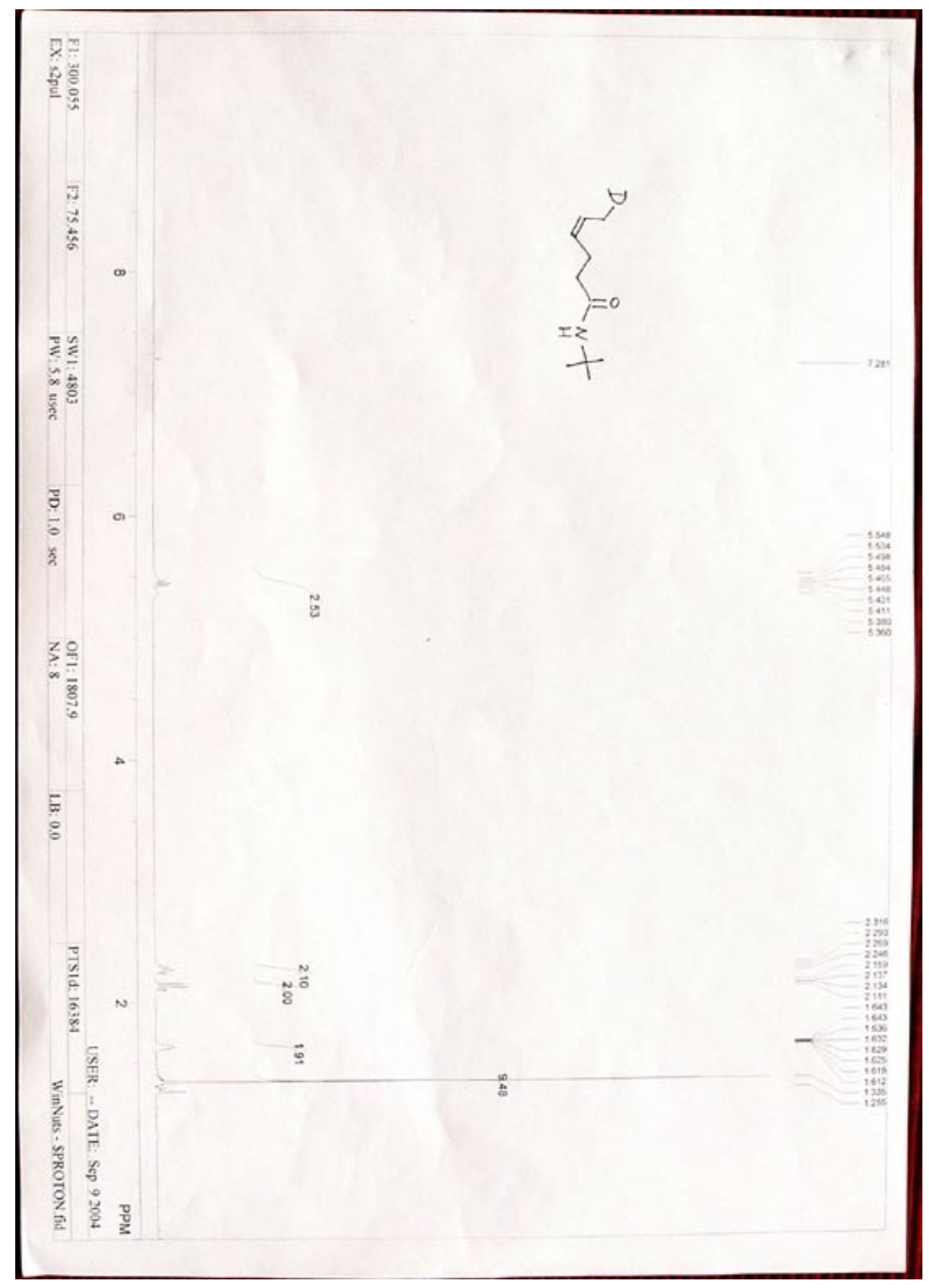




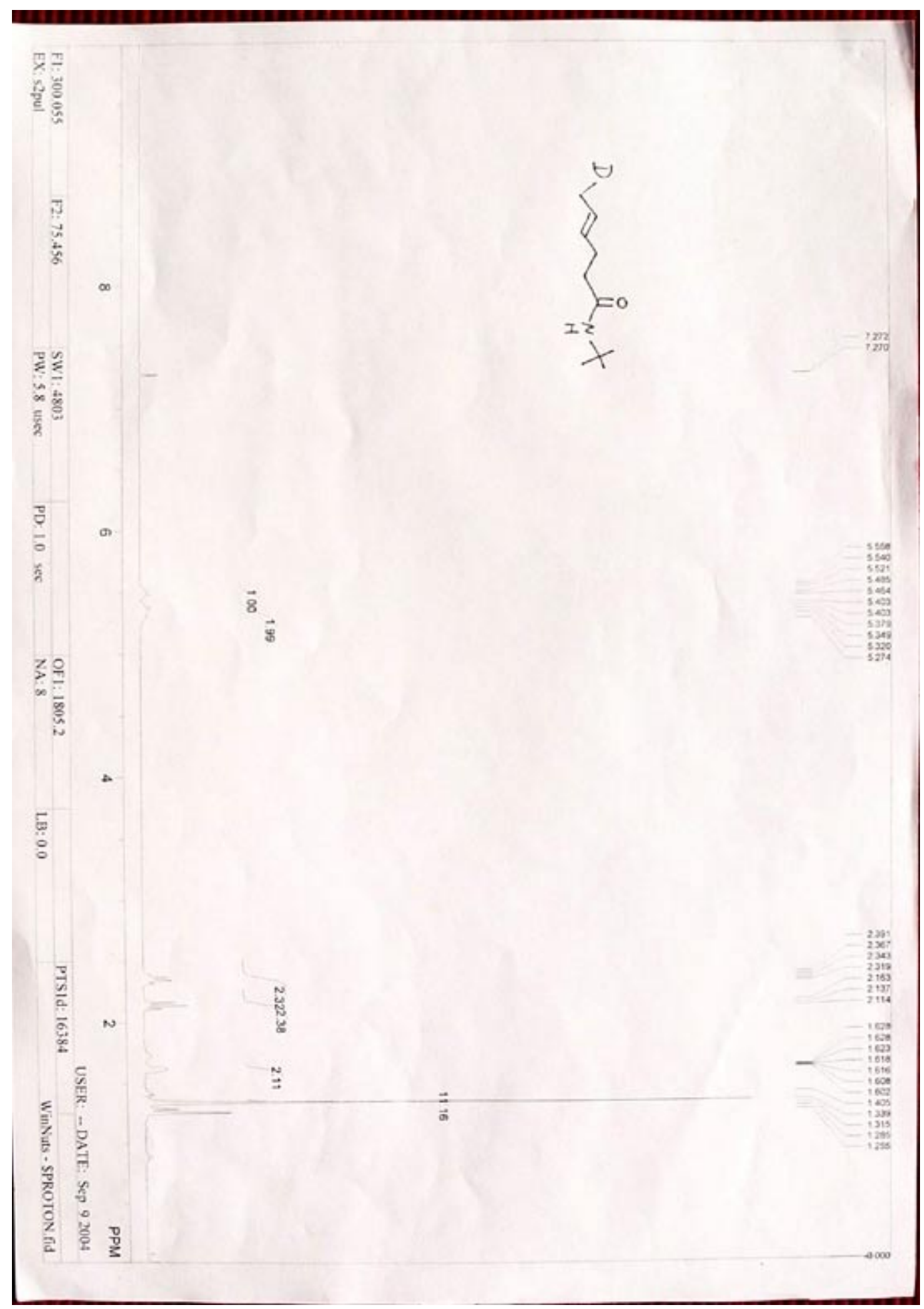




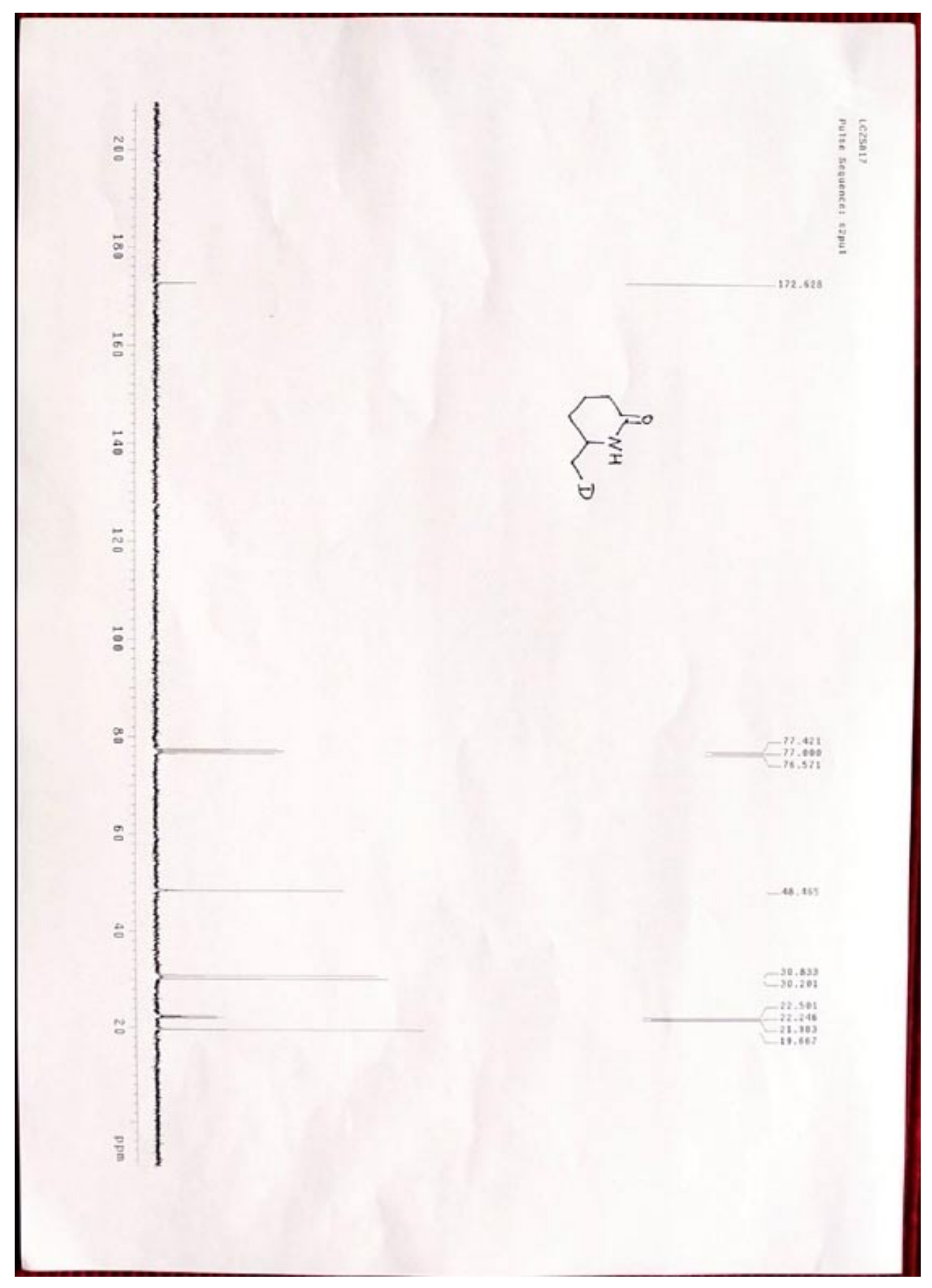

